\title{
The Effect of Phosphor Persistence on Image Quality in Digital X-ray Scanning Systems
}

by

\section{James Gordon Mainprize}

A thesis submitted in conformity with the requirements for the degree of Masters of Science Graduate department of Medical Biophysics University of Toronto

(C) Copyright James Gordon Mainprize 1997 
National Library

of Canada

Acquisitions and

Bibliographic Services

395 Wellington Street

Ottawa ON KIA ON4

Canada
Bibliothèque nationale

du Canada

Acquisitions et

services bibliographiques

395, rue Wellington

Ottawa ON KIA ON4

Canada
The author has granted a nonexclusive licence allowing the National Library of Canada to reproduce, loan, distribute or sell copies of this thesis in microform, paper or electronic formats.

The author retains ownership of the copyright in this thesis. Neither the thesis nor substantial extracts from it may be printed or otherwise reproduced without the author's permission.
L'auteur a accordé une licence non exclusive permettant à la Bibliothèque nationale du Canada de reproduire, prêter, distribuer ou vendre des copies de cette thèse sous la forme de microfiche/film, de reproduction sur papier ou sur format électronique.

L'auteur conserve la propriété du droit $d$ 'auteur qui protège cette thèse. $\mathrm{Ni}$ la thèse ni des extraits substantiels de celle-ci ne doivent être imprimés ou autrement reproduits sans son autorisation. 
To my Father

Who always said:

"You'll learn it in Grade 11 Physics" 


\title{
The Effect of Phosphor Persistence on Image Quality in Digital X-ray Scanning Systems
}

\author{
Jarnes Gordon Mainprize \\ Miasters of Science, 1997 \\ Department of Medical Biophysics \\ University of Toronto
}

\begin{abstract}
A digital $x$-ray scanning system offers several advantages over conventional film-screen systems. However. there are sources of image degradation resulting from the scanning motion. One type of scanning motion blur is due to the temporal response of the phosphor. This mechanism produces an asymmetrical blur, requiring the use of the complex optical transfer function (OTF) for correct characterization of image resolution. The luminescence response of eight phosphors was measured under pulsed $x$-ray excitation. A weighted exponential model was used to represent the primary luminesence. The dominant luminescence lifetimes ranged from $2.7 \mu$ s for $\mathrm{Gd}_{2} \mathrm{O}_{2} \mathrm{~S}: \mathrm{Pr}$ to $558 \mu \mathrm{s}$ for $\mathrm{Gd}_{2} \mathrm{O}_{2} \mathrm{~S}: \mathrm{Tb}$. The long term response was also measured, monitoring significant increases in a slow form of luminescence known as afterglow. Afterglow was modeled by an inverse power law equation. Afterglow was found to be strong in at least two of the phosphors studied (ZnCdS:Ag and $\mathrm{YTaO}_{4}: \mathrm{Tm}$ ). In selecting a phosphor for a scanning system. it must satisfy several criteria. including a fast temporal response. Thus, a phosphor like $\mathrm{Gd}_{2} \mathrm{O}_{2} \mathrm{~S}$ : Tb, which has a slow luminescence, but otherwise excellent imaging properties, may not be as useful as a more rapid phosphor like CsI:TI.
\end{abstract}




\section{Acknowledgements}

I would like to thank the following people who helped, encouraged and supported me:

- my supervisor Dr. Martin Yaffe and members of my supervisory committee Dr. John Rowlands and Dr. Stuart Foster;

- John Sabol and Gord Mawdsley for their invaluable efforts in maintaining the venerable x-ray generator used in my experiments;

- Brian Starkoski, who help me design the electronics required for the experimental apparatus;

- the students in Martin's group, Jeff, Justin, Rebecca and Normand, and the technicians, Roman, Robert, Jeff C., Betty, and Kim;

- M. Tecokotsky for providing the $\mathrm{Gd}_{2} \mathrm{O}_{2} \mathrm{~S}: \mathrm{Pr}$ samples, and D. Trauernicht of Kodak Inc for the Kodak phosphors, and the people at MCI-Optonix for their phosphor samples. 


\section{Contents}

$\begin{array}{ll}\text { Dedication } & \text { ii }\end{array}$

Abstract $\quad$ iii

Acknowledgements

List of Figures vii

List of Tables viii

List of Symbols $\quad$ ix

List of Abbreviations $\quad$ xi

Chapter 1 Introduction 1

1.1 Radiography ........................ 1

1.1.1 Limitations of Conventional Radiography . . . . . . . . . . 2

1.2 Quantifying Image Quality . . . . . . . . . . . . . . . . . . 5

1.3 Digital Radiography: Phosphor Coupled Systems . . . . . . . . . . . 7

1.3.1 Slot Scanning System . . . . . . . . . . . . . . . 8

1.3.2 Charge Coupled Devices and Time Delay Integration . . . . . . . . . 9

1.3.3 Image Quality Limitations of a TDI Slot Scanned System . . . . . . . . . 13

1.4 Luminescence . . . . . . . . . . . . . . . . . . 15

1.4.1 Fluorescence, Phosphorescence, and Afterglow . . . . . . . . . 17

1.5 Techniques for Measurement of Phosphor Luminescence Response . . . . . . . . . 20

1.6 Summary and Outline of Thesis . . . . . . . . . . . . . . . 24

Chapter 2 Effect of Phosphor Persistence on Resolution 26

2.1 Introduction . . . . . . . . . . . . . . . 26

2.2 Background and Theory . . . . . . . . . . . . . . . . 27

2.2 .1 Luminescence in a Scanning System . . . . . . . . . . . 27

2.2.2 Curve Fitting of the Luminescence Decay . . . . . . . . . . . . 30

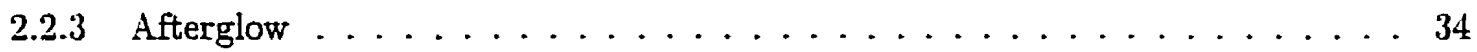

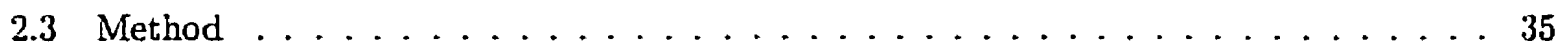




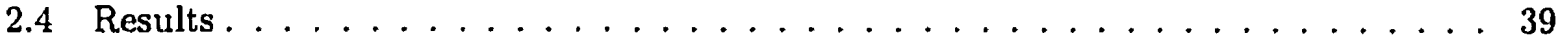

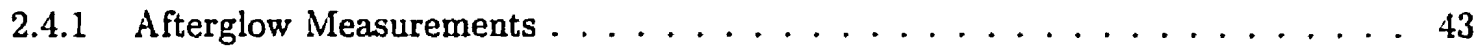

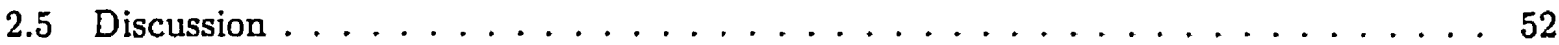

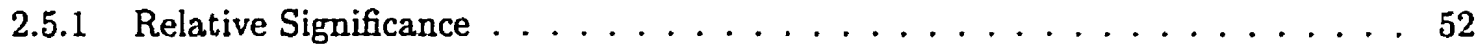

2.5 .2 Afterglow Revisited . . . . . . . . . . . . . . . . 53

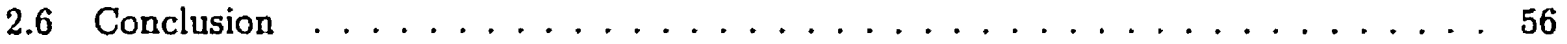

$\begin{array}{llc}\text { Chapter } 3 & \text { Future Work and Summary } & 58\end{array}$

3.1 Phosphors for Scanning Systems . . . . . . . . . . . . . . 58

3.2 Effect of Phase Shift on Image Quality in X-ray Imaging . . . . . . . . . . . 61

3.3 Phosphor Alternatives-Direct Conversion Systems . . . . . . . . . . . . 64

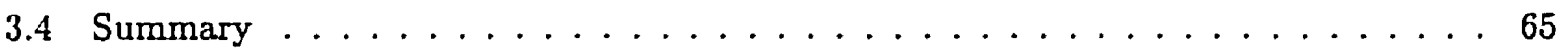

$\begin{array}{lll}\text { Appendix A } & \text { Description of Some Common Phosphors } & 67\end{array}$

Appendix B Levenberg-Marquhardt Non-Linear Least Squares $\quad 70$

B.1 Model Equations for Luminescence . . . . . . . . . . . . . . . 74

$\begin{array}{lr}\text { Bibliography } & 76\end{array}$ 


\section{List of Figures}

1.1 Elements of a conventional film-screen system . . . . . . . . . . 2

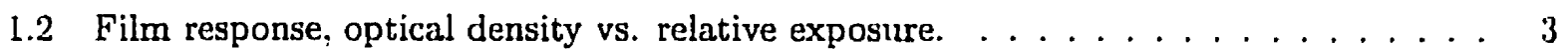

1.3 Distortion of square wave signal by the phase transfer function $\ldots \ldots \ldots \ldots$

1.4 Example of large area detector $\ldots \ldots \ldots \ldots \ldots$

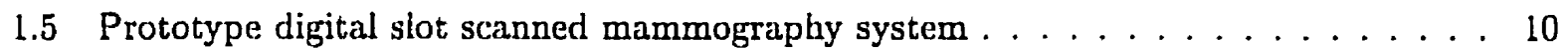

1.6 Charge flow in a CCD . . . . . . . . . . . . . . . 10

1.7 Charge transfer in a CCD . . . . . . . . . . . . . . . . . 12

1.8 An energy level diagram showing fluorescence, phosphorescence. and afterglow . . 18

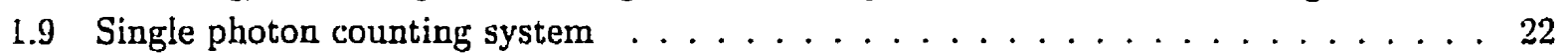

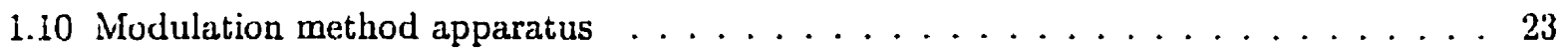

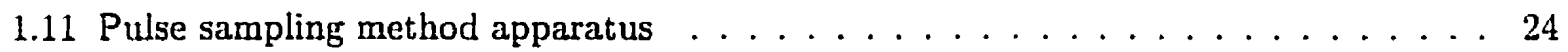

2.1 TDI Charge transfer under $\mathrm{x}$-ray irradiation $\ldots \ldots \ldots \ldots \ldots \ldots \ldots$

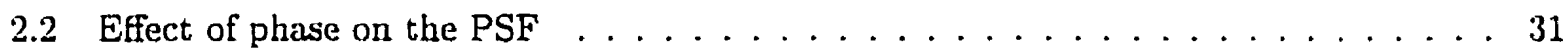

2.3 Phosphor response to long $\mathrm{x}$-ray pulse $\ldots \ldots \ldots \ldots \ldots \ldots \ldots \ldots$

2.4 A schematic of the pulse sampling experiment . . . . . . . . . . 36

2.5 Comparison of predicted and measured noise $\ldots \ldots \ldots \ldots \ldots \ldots \ldots \ldots$

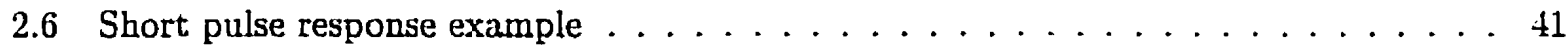

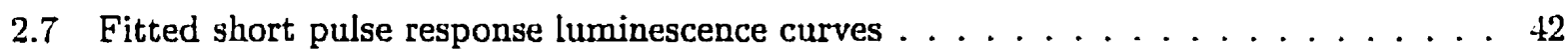

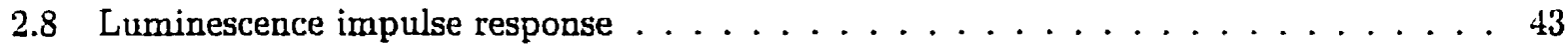

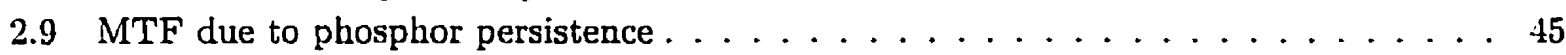

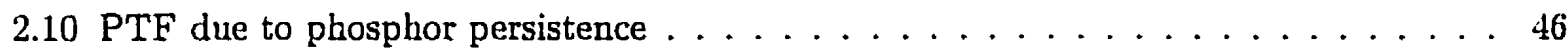

2.11 Long pulse response measurements $\ldots \ldots \ldots \ldots \ldots \ldots \ldots \ldots \ldots \ldots \ldots$

2.12 Comparison of long pulse response to literature $\ldots \ldots \ldots \ldots \ldots \ldots$

2.13 Afterglow regression curves for 4 phosphors . . . . . . . . . . . . . 49

2.14 Comparison of MTF obtained from short and long pulse luminescence response . . 51

2.15 Geometry of compressed breast . . . . . . . . . . . . . . . 56

3.1 Phase distortion of high and low resolution systems $\ldots \ldots \ldots 63$ 


\section{List of Tables}

2.1 Phosphors tested and their properties .................. 40

2.2 Fitted parameters for the phosphors tested $\ldots \ldots \ldots \ldots \ldots$

2.3 Lifetime measurements from literature . . . . . . . . . . . . . . . 44

2.4 Afterglow times . . . . . . . . . . . . . . . . . . . 50

2.5 Regression parameters obtained from the inverse power law model for afterglow . . . 51

2.6 Comparison of various blurring mechanisms . . . . . . . . . . . 53 


\section{List of Symbols}

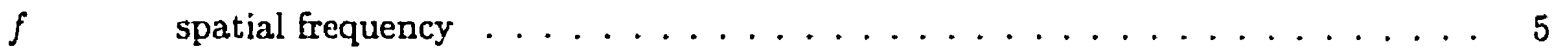

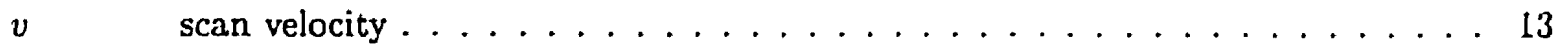

$\Delta v \quad$ scan velocity/clock rate mismatch $\ldots \ldots \ldots \ldots \ldots \ldots$

$n_{\text {rous }}$ number of pixels in a CCD row $\ldots \ldots \ldots \ldots \ldots \ldots$

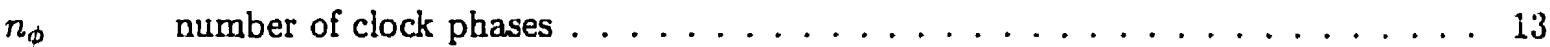

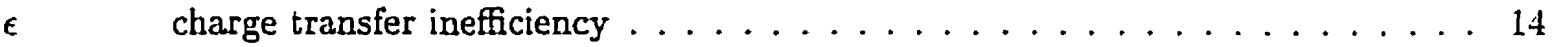

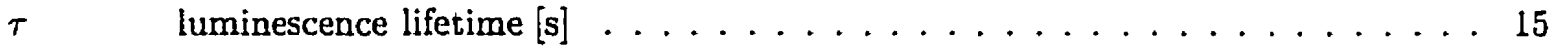

$\alpha \quad$ electron transition probability rate $\left[s^{-1}\right] \ldots \ldots \ldots \ldots$

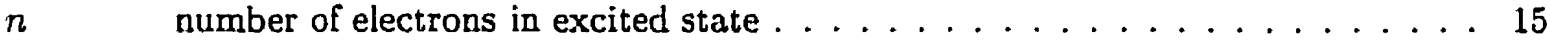

$w_{j} \quad$ relative weighting of the $j$ th exponential component $\ldots \ldots \ldots 16$

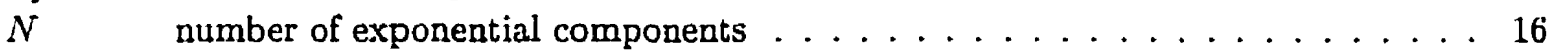

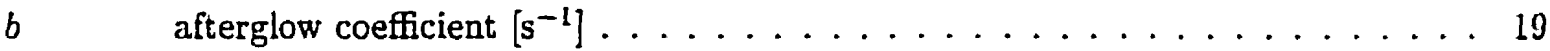

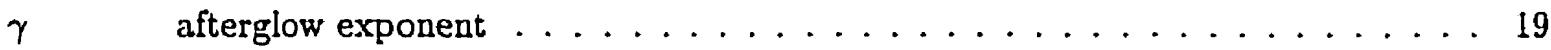

$O T F_{p}(f)$ OTF component for primary luminescence ................ 29

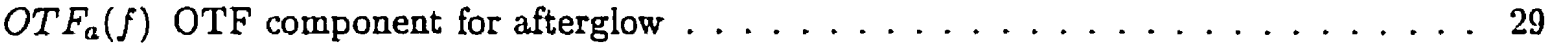

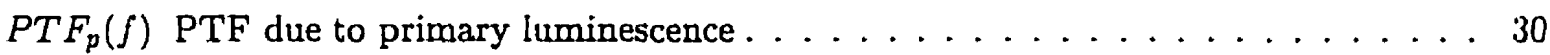

$M T F_{p}(f) \mathrm{MTF}$ due to primary luminescence $\ldots \ldots \ldots \ldots \ldots \ldots$

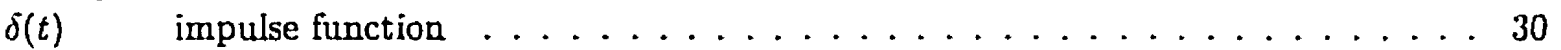

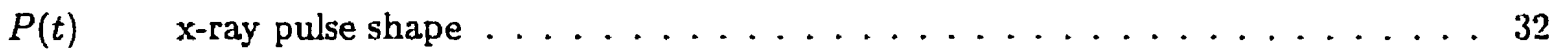

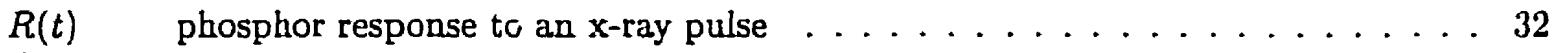

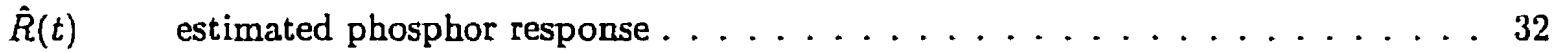

$\chi^{2} \quad$ chi-square use in least squares regression $\ldots \ldots \ldots \ldots \ldots \ldots$

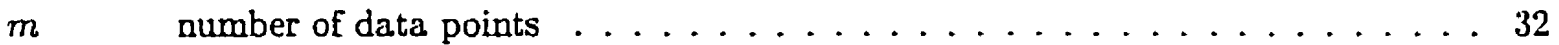

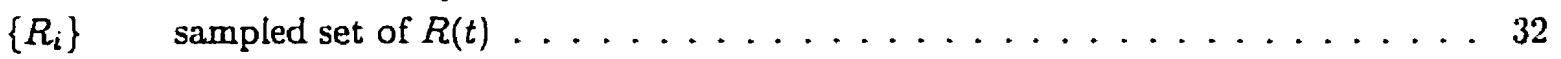

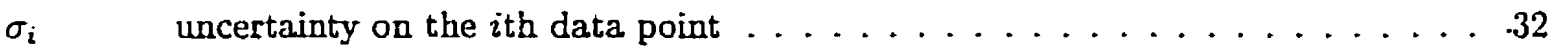

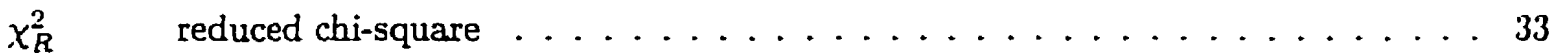

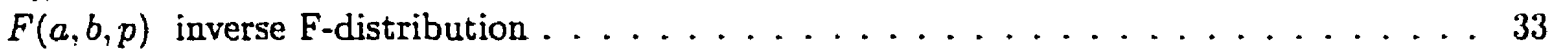

$R_{T}(t) \quad$ phosphor response to an $\mathrm{x}$-ray pulse (primary + afterglow) $\ldots \ldots \ldots 34$

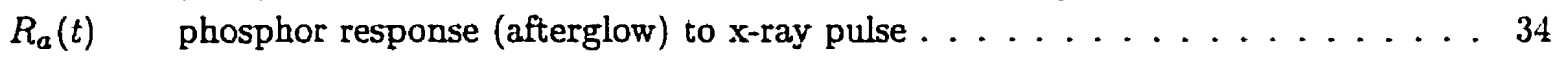

$\beta \quad$ ratio of primary luminescence to total luminescence $\ldots \ldots \ldots \ldots$

c current-voltage amplifier gain $[\mathrm{V} / \mathrm{A}] \ldots \ldots \ldots \ldots \ldots \ldots$

e elementary electron charge $[\mathrm{C}] \ldots \ldots \ldots \ldots \ldots \ldots \ldots \ldots \ldots$

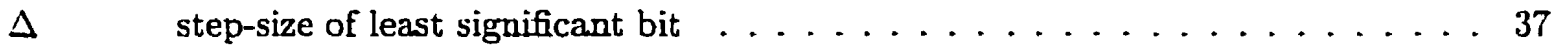


$N_{\text {avg }} \quad$ number of traces averaged $\ldots \ldots \ldots \ldots \ldots \ldots \ldots$

$T \quad$ integration time constant of amplifier $[\mathrm{s}] \ldots \ldots \ldots \ldots \ldots$

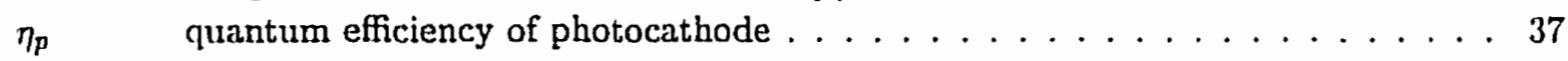

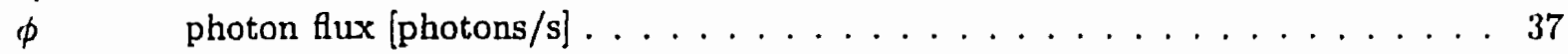

$g \quad$ PM'T electron multiplication factor [electrons/photon] $\ldots \ldots \ldots \ldots$

$\sigma_{\eta} \quad$ noise on PMT gain [electrons/photon] $\ldots \ldots \ldots \ldots$

$s \quad$ standard deviation (width) of Gaussian PSF $[\mu \mathrm{m}] \ldots \ldots \ldots$ 


\section{List of Abbreviations}

TDI time-delay integration $\ldots \ldots \ldots \ldots \ldots \ldots$

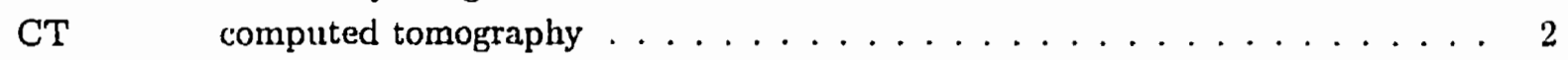

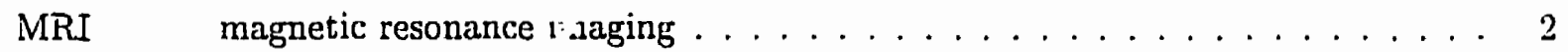

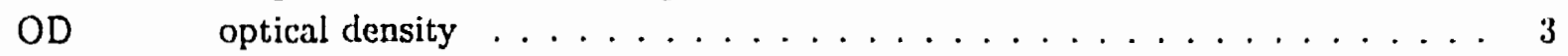

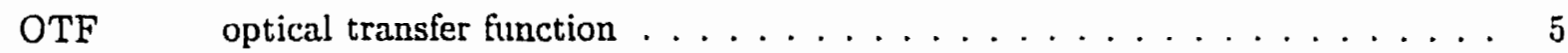

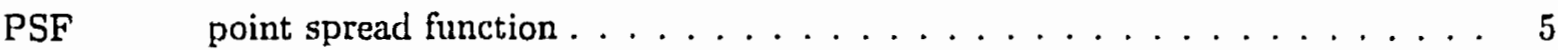

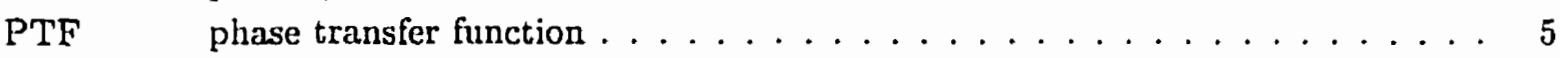

MTF modulation transfer function $\ldots \ldots \ldots \ldots \ldots$

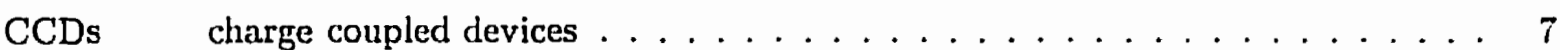

$\mathrm{S} / \mathrm{P} \quad$ scatter to primary ratio $\ldots \ldots \ldots \ldots \ldots \ldots$

EHP $\quad$ electron-hole pairs $\ldots \ldots \ldots \ldots \ldots \ldots$

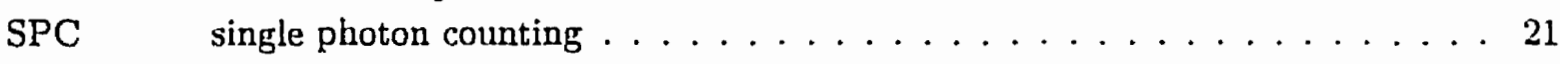

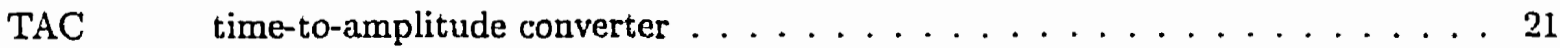

BGO bismuth germanate $\mathrm{Bi}_{4} \mathrm{Ge}_{3} \mathrm{O}_{12} \ldots \ldots \ldots \ldots \ldots \ldots \ldots \ldots \ldots \ldots \ldots \ldots \ldots$

BaFCl:Eu barium fluoro-chloride (europium doped) . . . . . . . . . . . 40

CsI:Tl cesium iodide (thallium doped $) \ldots \ldots \ldots \ldots$

$\mathrm{Gd}_{2} \mathrm{O}_{2} \mathrm{~S}: \operatorname{Pr}$ gadolinium oxysulphide (praseodymium doped) $\ldots \ldots \ldots \ldots$

$\mathrm{Gd}_{2} \mathrm{O}_{2} \mathrm{~S}$ : $\mathrm{Tb}$ gadolinium oxysulphide (terbium doped) . . . . . . . . . . . . . . . . . 40

LaOBr:Tm lanthanum oxybromide (thulium doped) . . . . . . . . . . . . . 40

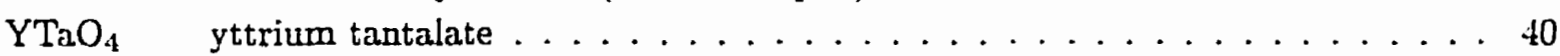

$\mathrm{ZnCdS:Ag} \mathrm{zinc} \mathrm{cadmium} \mathrm{sulphide} \mathrm{(silver} \mathrm{doped)} \mathrm{\ldots ................} 40$

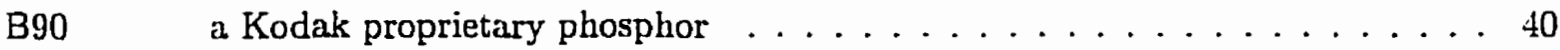

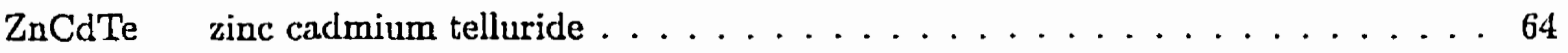




\section{Chapter 1}

\section{Introduction}

Digital acquisition and processing of radiographic images offer potential improvements in image quality and in the flexibility of image display and transmission. A key challenge is the development of high quality $\mathrm{x}$-ray detectors for digital radiography. Several different designs have been proposed, many using an $x$-ray absorbing phosphor that emits light when irradiated, coupled to an electronic light-sensitive detector to produce the digital image. A specific approach, which is cost effective and clinically feasible, involves a slot scanning design in which the image is acquired by timedelay integration (TDI). Because it is a scanning technique, the temporal response of the detector can potentially affect image quality. Specifically, the temporal response of luminescence from the phosphor may cause blurring of the acquired image. To determine the amount of blurring, the luminescence response of the phosphor must be carefully measured and the mechanism by which the components of luminescence affect the image must be understood.

\subsection{Radiography}

Conventional radiography is illustrated in Figure 1.1. The imaging system consists of an $x$-ray tube and a light-tight cassette containing photographic film and a phosphor screen. The cassette is placed on the opposite side of the object being imaged. In passing through the body, $x$-rays are 
differentially absorbed by its tissue components. In the light-tight cassette, the transmitted $x$-rays impinge on a phosphor screen which emits light that, in turn, exposes the film. Processing the film reveals the image which is a two dimensional projection of $\mathrm{x}$-ray transmission through the patient.

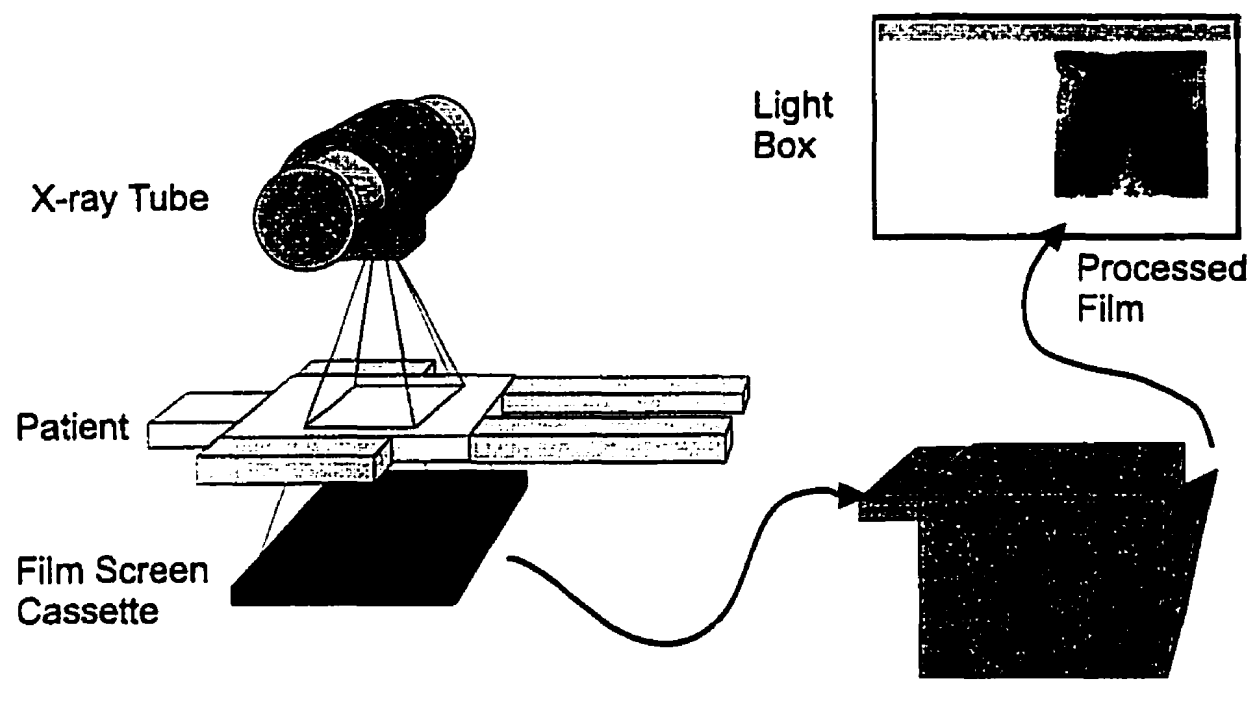

Film Developer

Figure 1.1: Elements of a conventional film screen-system.

Despite the development of modern imaging modalities, such as computed tomography (CT), ultrasound imaging, and magnetic resonance imaging (MRI), conventional radiography is still the most common form of diagnostic imaging $[1,2]$. There are several reasons for its continued use. Xray imaging can achieve very high resolution ( $>10$ line-pairs $/ \mathrm{mm}$ ) making it well suited for specific imaging tasks such as mammography and bone radiography. In addition, $\mathbf{x}$-ray facilities are well established and are readily available. An x-ray radiography system is also of lower cost compared to more technically sophisticated techniques like CT or MRI.

\subsubsection{Limitations of Conventional Radiography}

Despite its obvious strengths, film-screen radiography has demonstrated several limitations in image quality. Some of these limitations include: 


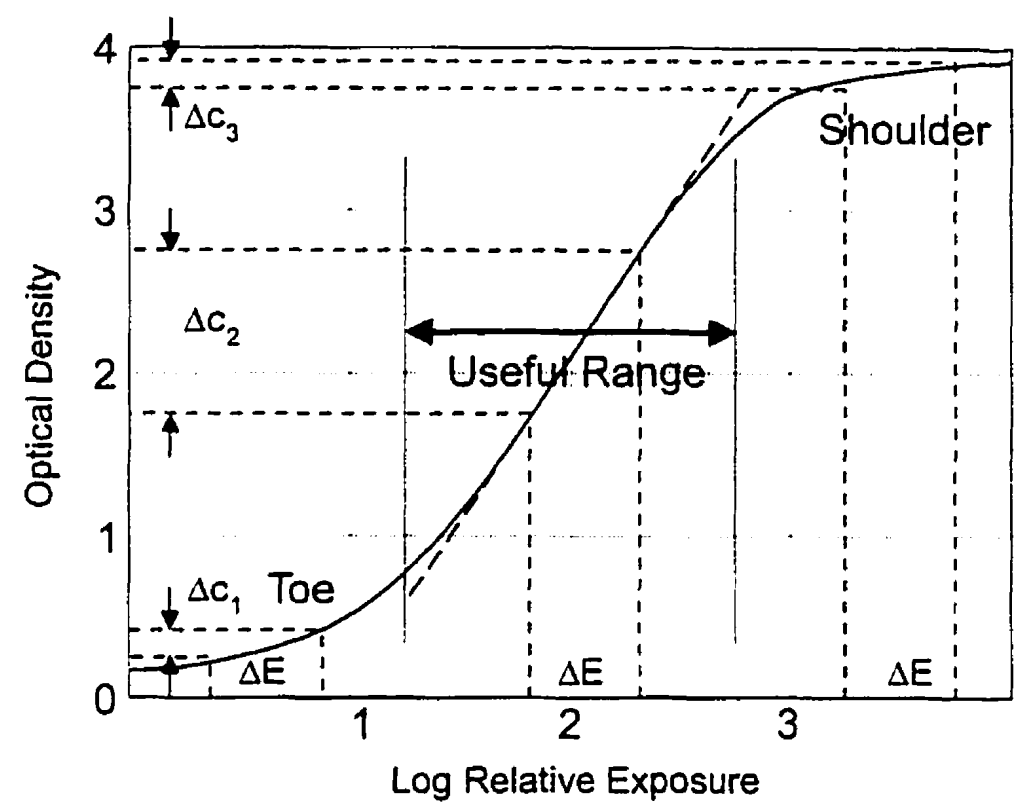

Figure 1.2: A plot of a typical radiographic film response. The optical density (OD), or "blackness" of the film is plotted as a function of log relative $x$-ray exposure. Note that in the regions of the toe and shoulder, the contrast, $\Delta c$, is reduced for the same change in log exposure, $\Delta E$

- Non-linear Film Response: Film-screens typically exhibit a response as a function of x-ray signal (or exposure) as shown in the sketch Figure 1.2. Regions of an image that correspond to exposures that fall in the toe or shoulder of the response curve have severely reduced contrast, and subtle lesions may be missed. Because of this, the useful range over which contrast is good is quite limited. For example, a typical mammographic film-screen combination has a useful range of a factor of $25[3]$.

Light Diffusion: The light emission from the point of $x$-ray interaction in the phosphor can occur in any direction. When the light reaches the surface of the phosphor, it can be displaced some distance laterally from its original point. Because of the spread in light, the resultant image will be blurred. The amount of blurring will increase with screen thickness. Although thicker screens improve $x$-ray absorption, they allow light to diffuse further from the site of $\mathrm{x}$-ray interaction. 
- Noise Limitations: Radiographic film consists of small grains of silver halide that are sensitive to light. Variations in the grain size and random distribution of the grains are a major source of noise in the resulting image. This is often referred to a grain noise[4]. The lighi emitted from the phosphor screen can be affected by variations in screen structure which is also a potential source of noise[5].

- Recording and Display: The film acts as both the recording medium and the display medium. Simultaneous optimization of both tasks is difficult and involves tradeoffs between them.

- Exposure Errors/Recalled Exams: If an error is made in sclecting the $x$-ray settings for the exam, the image may be over-exposed, under-exposed, too noisy, or blurred. This may result in the patient being recalled for a repeat procedure.

- Processing Artefacts: Film processing can cause streaking produced by the film transport rollers, dust specks, static discharge patterns from low humidity, and blurring due to poor screen-film contact[6]. These artefacts are distracting and can potentially obscure important anatomical information. Very careful quality assurance is required to maintain reproducible film quality.

Because of the limitation of conventional radiography, alternative acquisition techniques are being investigated that utilize a digital image acquisition system. A digital radiography system uses a device that can record the $x$-ray signal from a small area and produce an electronic signal that is proportional to the $x$-ray intensity.

Digital $x$-ray systems have the benefit of increased dynamic range[ $7,8,9]$, the ability to perform image enhancement to improve the salient features of the image[10], and the potential to perform computer aided diagnosis[11, 10]. Because of these advantages, digital systems are being rapidly developed for clinical use.

Before some digital systems can be described, the concepts which allow imaging systems to be compared must be introduced. 


\subsection{Quantifying Image Quality}

In order to compare imaging systems, a measure is required to quantify some of the aspects of image quality. Based on linear systems theory, an imaging system can be characterized by determining its optical transfer function (OTF). The OTF describes how the imaging system responds to an input signal as a function of spatial frequency, $f$. It can be shown[12] that the OTF is the Fourier transform of the imaging system's response to an impulse function, $\delta(x, y)$. An image produced from an impulse function input is known as the point spread function (PSF). Hence, the OTF is the following:

$$
O T F\left(f_{x}, f_{y}\right)=\mathcal{F}\{\operatorname{PSF}(x, y)\}
$$

where $f_{x}$ and $f_{y}$, and are spatial frequencies in the $x$ and $y$ direction, expressed as inverse distance $\left(\mathrm{mm}^{-1}\right)$. Using linear systems theory, the OTF is a powerful tool that allows the analysis of an imaging systern by breaking the system into a cascade of stages:

$$
O T F(f)=O T F_{1}(f) \cdot O T F_{2}(f) \cdot O T F_{3}(f) \cdot \ldots
$$

Thus, individual stages can be characterized as well as the overall system. Based on knowledge obtained from the OTF of one stage, that stage can be optimized for best imaging performance.

In general, the OTF is a complex function with real and imaginary components. The OTF is often separated into magnitude and phase components referred to as modulation transfer function (MTF) and phase transfer function (PTF). The MTF is defined as the magnitude of the OTF relative to the OTF at zero spatial frequency:

$$
\operatorname{MTF}\left(f_{x}, f_{y}\right)=\frac{\left|O T F\left(f_{x}, f_{y}\right)\right|}{|O T F(0,0)|} .
$$

The MTF describes how well the relative amplitude of spatial frequency components of an object are preserved. In the context of radiological imaging, the difference in intensity, or contrast, 
is the basis by which structures can be observed. The MTF can reveal how much contrast will be maintained by an imaging system, according to the size of the structure of interest.

The PTF is defined as the argument of the complex value of the OTF. The argument is the angle between the positive real axis and a complex number plotted as a vector on the complex plane[13]:

$$
\operatorname{PTF}\left(f_{x}, f_{y}\right)=\arg \left(O T F\left(f_{x}, f_{y}\right)\right) \text {. }
$$

If the PSF is measured to be symmetric, then the OTF will be a purely real function and the MTF will be sufficient in characterizing the imaging system. When the PSF lacks symmetry, then the PTF must also be considered.
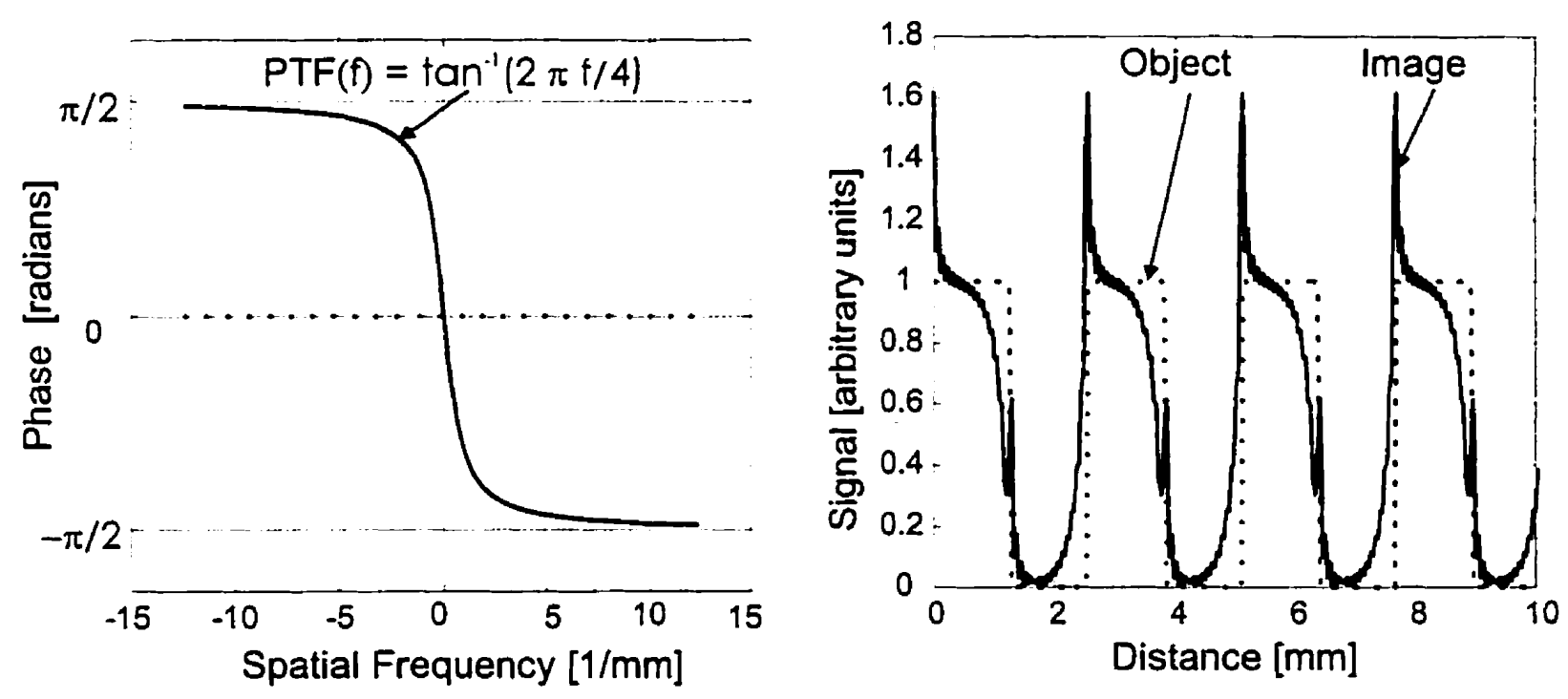

Figure 1.3: Comparison of an ideal square wave signal (dotted line, zero phase shift) and the distorted signal (solid line) resulting from applying the phase transfer function, $P T F(f)$ to a square wave

The PTF represents distortions in the image. These distortions occur because information at different spatial frequencies is shifted in phase. For example, if an imaging system exhibits a PTF like the one illustrated on the left of Figure 1.3, the resultant image of a square wave train will be 
dramatically altered, as shown on the right. Note that in real imaging systems, the PTF is always combined with amplitude modulating effects, and the distortions are not quite as pronounced as shown in Figure 1.3. To reduce distortions of this nature, most imaging systems are designed to produce symmetric (or very nearly symmetric) PSF.

\subsection{Digital Radiography: Phosphor Coupled Systems}

One type of digital system under development employs a phosphor to convert the $\mathrm{x}$-ray energy to light (visible) photons. These photons are, in turn, detected by a light sensitive electronic device in which a charge signal is produced that is proportional to the light intensity. In this type of system, the conventional geometry of Figure 1.1 is used. However, the phosphor coupled detector replaces the film-screen cassette.

There are several types of phosphor coupled systems using different detector designs. Some detectors are large area devices which consist of a large array of photosensitive elements. These arrays may be composed of a single large active matrix imager composed of amorphous silicon[14] or by combining multiple smaller area detectors called CCDs(charge coupled devices)[10]. Figure 1.4 shows this type of detector. There are some difficulties with these types of systems. Because of the enormous number of pixels required for a large area detector, there is a correspondingly large number of readout lines and the electronics required for readout must be very complex. As well. sensitivity variations may occur from pixel to pixel, and need to be corrected by calculationintensive computer algorithms. Alternatively, slot shaped detectors can be employed in a scanning system. These devices may be a linear photodiode arrays[15], or narrow slot style CCDs[16]. The number of detector elements are significantly reduced compared to large area detectors, relaxing the demands on the detector technology. 


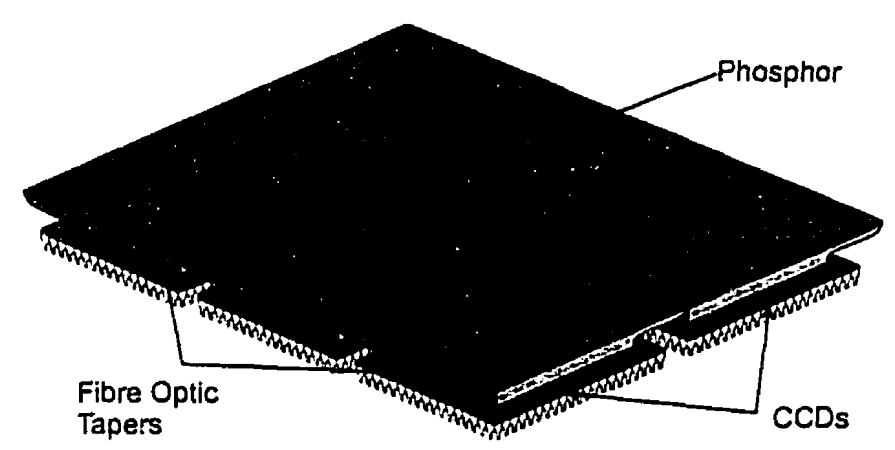

Figure 1.4: This is an example of a large area phosphor coupled detector. This detector is made from multiple small area CCDs. The light from the phosphor is coupled to the photosensitive CCD by a fibre optic taper.

\subsubsection{Slot Scanning System}

In a slot scanning $\mathrm{x}$-ray system, a long narrow detector scans across the patient in synchrony with a collimated $x$-ray beam. A slot scanning system has several advantages over a full sized twodimensional detector array. The detector is clearly much smaller and, hence, much easier to design. As well, the narrow slot geometry provides a dose-efficient scattered radiation rejection. Scattered $\mathrm{x}$-rays adds a signal across the image that contains no location information (there is no way to tell the specific originating point of a scatter signal) and reduces contrast across the image. For example, the relative amount of scattered radiation to primary (unscattered) radiation (S/P) in a breast exam can typically range between 0.3 and 1.0 which reduces contrast by 50 to $70 \%[17]$. Because scattered radiation. can be quite high, it is very important to eliminate its detection. In the slot scan system, much of the scatter simply does not hit the detector. For example, using a 9 mm slot can reduce scatter by $4-5$ times compared to a full area detector[18]. If the slot width is even smaller, the contrast reduction due to the detection of scattered radiation is negligible.

These advantages over full area detectors are gained at the expense of an increased imaging time and a very inefficient use of the output of the $x$-ray tube: most of the $x$-rays produced are absorbed by the lead blockers used to create the narrow slit of $x$-rays. The longer imaging times significantly increase the chance that patient motion will blur the image. Motion occurs as a 
result of involuntary muscle motion, such as the heart beating or peristalic motion of the intestinal tract, and semi-involuntary motion such as breathing. Thompson suggests that for imaging of the chest (heart and lungs) without motion blurring, exposure times should be limited to $10 \mathrm{~ms}$ or less[19]. For the head and the extremities, there is a much smaller range of motion, and exposure times up to one second should be acceptable. Even in mammography, with breath-hold and firm compression to immobilize the breast, motion can become a factor if the exposure time exceeds 1 s.[20] With a scanning system, the speed must be kept as high as possible to reduce blurring due to patient motion effects. Velocities reported for scanning $x$-ray systems range from $23 \mathrm{~mm} / \mathrm{s}$ for a CCD-based mammography system[21] to $219 \mathrm{~mm} / \mathrm{s}$ for a self-scanned photodiode array chest radiography system[15].

This laboratory is investigating a prototype design of a slot scanning phosphor coupled system that uses a scanning detector. It is designed specifically for mammography, which demands very high spatial resolution $\left(\approx 10 \mathrm{~mm}^{-1}\right)[3]$ and good contrast. A simple schematic of the system is shown in Figure 1.5. The detector consists of a strip of phosphor optically coupled by a fibre optic taper to three CCDs each consisting of a $2048 \times 64$ array of pixels. The pixel size at the imaging plane is approximately $42 \mu \mathrm{m}$.

\subsubsection{Charge Coupled Devices and Time Delay Integration}

A CCD is a device that is able to collect localized packets of charge and by manipulation of clock signal, it can transfer the charge to an amplifier and then to a read-out device such as an analogdigital converter. A simple schematic of charge transfer from pixel to pixel is shown in Figure 1.6.

When light strikes a CCD, the absorbed photons free electron-hole pairs (EHP). Depending on the design of the CCD, either the electrons or the holes will be collected in localized charge packets in a potential well. The potential wells are defined by applying different voltages to an array of clock lines that set up potential barriers. If a clock signal is set, charge can be collected in the well. By careful manipulation of the clock signals, the charge packets can be moved up or down 


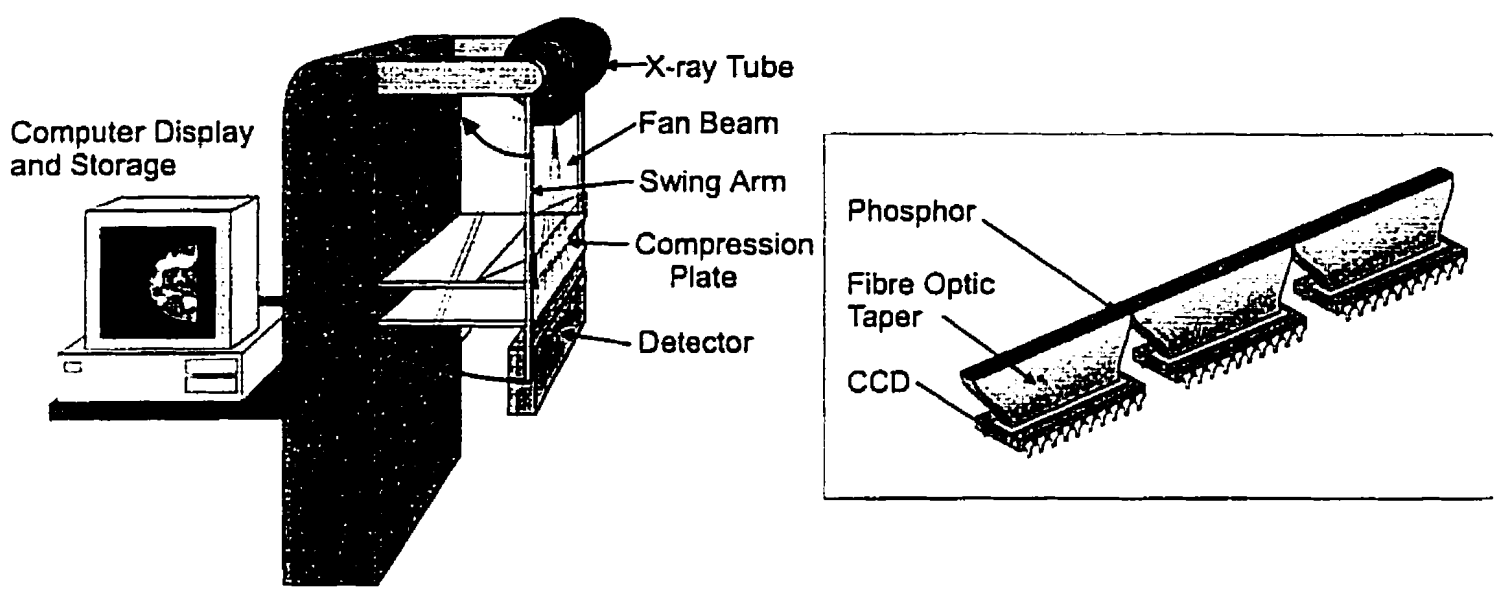

Figure 1.5: A schematic of a prototype digital slot scanned mammography system. The inset shows the components housed inside the detector assembly.

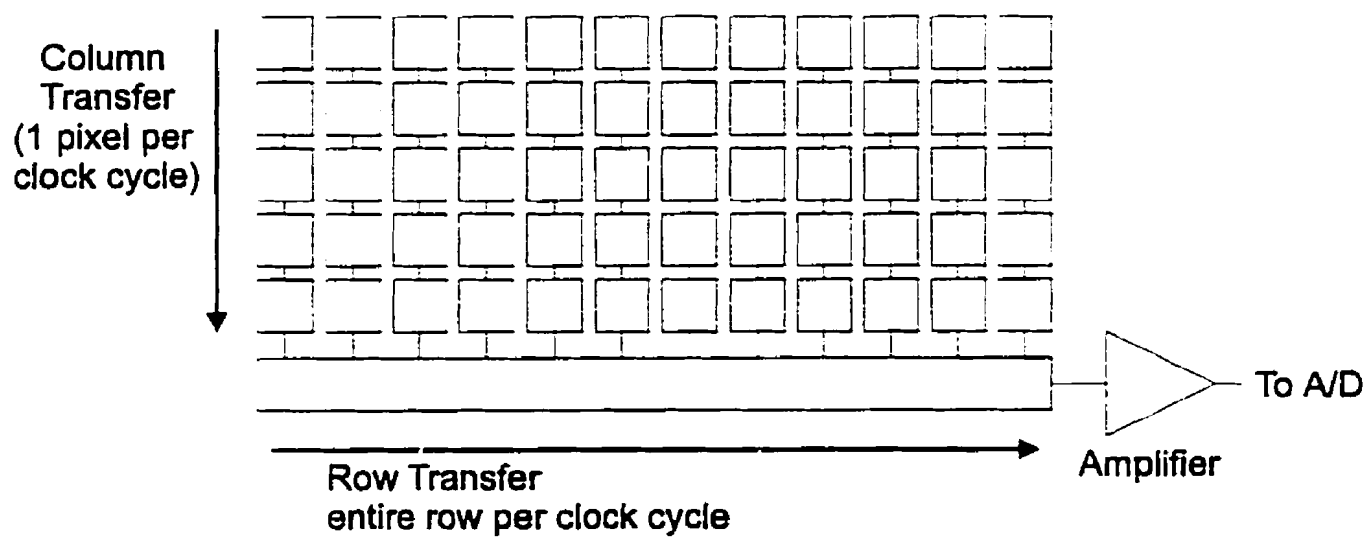

Figure 1.6: Schematic of the charge flow in a CCD. The charge packets are transferred down each column, one pixel per clock cycle. At the bottom an entire row is rapidly read-out to the A/D converter once per clock cycle. 
columns without any mixing of the charge from neighboring packets. Figure 1.7 shows a typical clock transfer scheme using four clock signals (four phases). The signals are applied to the clock lines on the surface of the CCD. For example at time $t=t 1$, the voltages are applied to clocks $\phi 2 \rightarrow \phi 4$. The freed charge is collected under these clock lines. The charge can then be moved along the CCD by changing the clock voltages. At $t=t 2$ the the voltage on $\phi 2$ is switched off and the charge packet flows underneath $\phi 3$ and $\phi 4$. At $t=t 3, \phi 1$ can be turned on, allowing the charge packet to move a little to the right. The clock voltages are continually cycled to move the charge down the column. Note that the charge packet is always separated by at least one clock line width, preventing mixing of the signals.

A CCD can be used in a stationary mode called "frame transfer mode". The CCD collects charge (charge integration) for a long time with the clock lines fixed at $t=t 1$. The illuminating source (light, $\mathrm{x}$-rays, etc) is shut off and the clocks signal are rapidly changed to shuffle the charge signals down the columns and sent out serially to the amplifier and digitized to form the pixel values of the image.

In a scanning system, a modification of this technique, called time delay integration (TDI), is used to acquire a large area image with a very narrow CCD. In contrast to frame-transfer, the TDI technique permits charge integration to occur at every stage $(t 1, t 2, t 3, \ldots)$ in the clock cycle. The CCD is set to scan mechanically across the object being imaged at a constant velocity. At the same time, the clock signals are sent to the CCD to make the charge packets transfer down the columns at an equal velocity in the opposite direction. This localizes the charge packets corresponding to a specific location beneath the object, and charge produced by light can build up in this packet. Integration time for the charge packet has been increased by a factor equal to the number of rows in the CCD over a single row detector scanning at the same velocity. This increased integration time yields an enhanced photosensitivity and signal-to-noise ratios. As well, inter-pixel uniformity is improved because of the effect of averaging the response of several rows of pixels. 

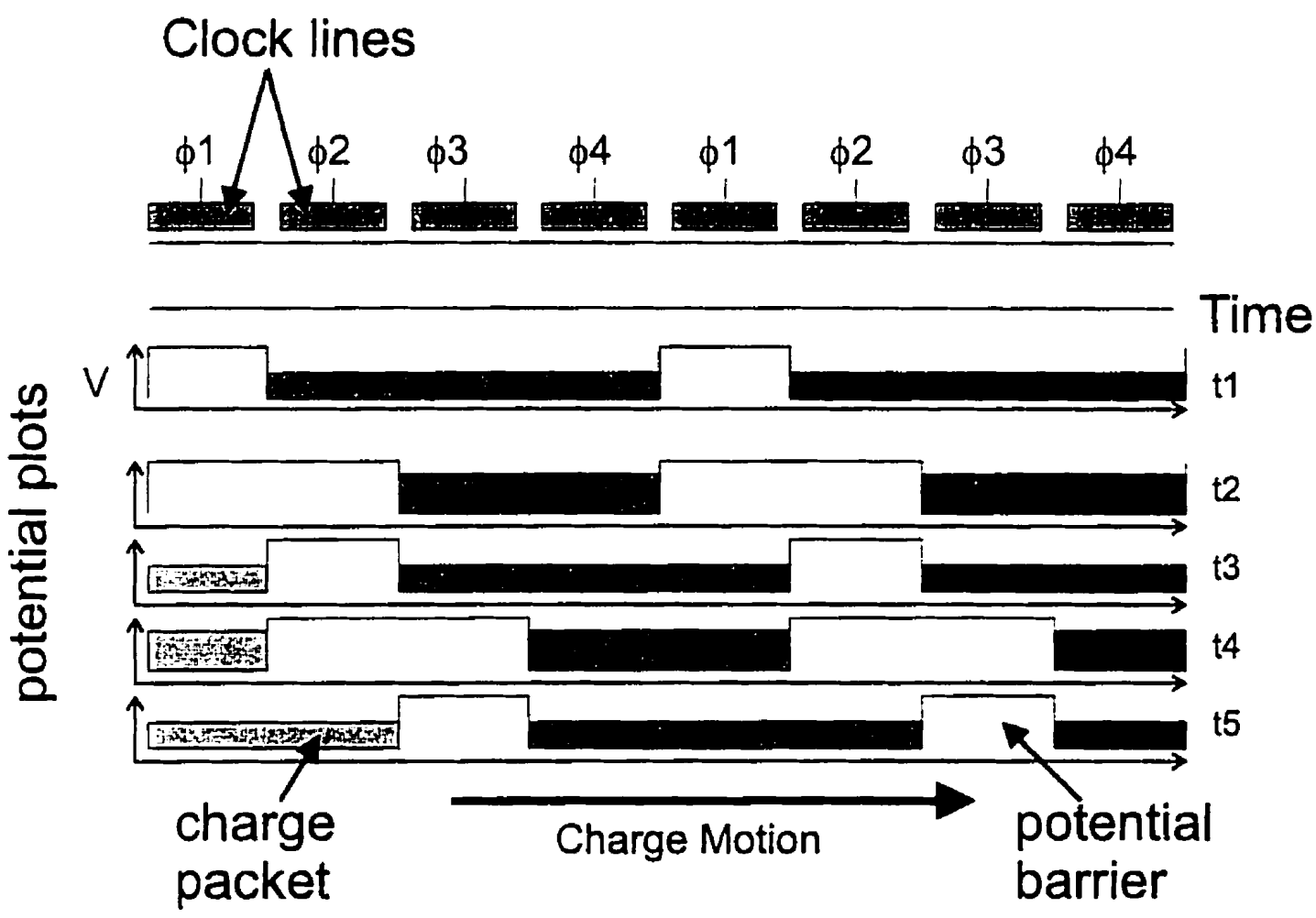

Figure 1.7: Charge transfer in a CCD. Voltages applied to clock lines $(\phi 1, \phi 2$, etc) produce an electric field in the bulk of the CCD. The electric field acts to produce potential barriers that localize the charge, represented by the dark bands in the voltage diagrams. At different times $(t 1, t 2, t 3, .$.$) in the clock cycle, the voltages on the clock lines are changed. As the potential barriers$ are toggled, the charge packets are able to move to adjacent regions. By repeating this cycle, the charge can be made to move down a column. 


\subsubsection{Image Quality Limitations of a TDI Slot Scanned System}

The design of the slot scanning system imposes several challenges to image quality. Maidment et al.[16], in a detailed analysis of a mammography slot scanning system identified several of these limitations. Some of the resolution limitations are similar to those found in film-screen systems. These include focal spot blurring, spatial light spread in the phosphor, and geometrical blurring.

In addition, there have been several investigations $[16,22,23]$ which indicate that there are image quality limitations unique to the slot-scanned system. These occur in the scan direction and are associated with the temporal response of the detector components as a result of the TDI technique. The three dominant temporal effects are velocity-scan rate mismatching, clock phasing, and charge lag. As well, the temporal response of the phosphor must be considered.

\section{Velocity-Scan Rate Mismatch}

If there is a mismatch between the mechanical scanning velocity and the charge transfer rate, the result is a smear across the image, reducing the $\operatorname{MTF}[22,16]$ :

$$
M T F_{V}(f)=\operatorname{sinc}\left(f \frac{\Delta v}{v} n_{\text {row }}\right)
$$

where $v$ is the scanning velocity, $\Delta v$ is the difference between the scanning velocity and the charge transfer velocity, and $n_{\text {row }}$ is the number of rows of pixels in the CCD in the TDI direction. For a CCD with 64 rows, velocity mismatches as low as $1 \%$ can cause a $7 \%$ loss in signal at $10 \mathrm{~mm}^{-1}$.

\section{Clock Structure}

A second source of blur is due to the finite number of clock phases[24]. During the clock cycle, the charge is transferred at discrete times, rather than a smooth continuous movement of charge. Thus, each detector element sweeps out an area that is slightly larger than the nominal pixel pitch of the CCD in the scan directirn. If $L$ is the nominal pixel pitch of the CCD (stationary) and $n_{\phi}$ 
is the number of clock phases (usually between 2 and 4), then the resolution loss due to the clock structure can be described as follows:

$$
M T F_{C S}(f)=\operatorname{sinc}\left(\frac{f L}{n_{\phi}}\right) .
$$

\section{Charge Transfer Inefficiency}

Another biurring mechanism is due to incomplete charge transfer between adjacent wells. As the charge packets ripple down a column, some of the charge is left behind. This remaining charge is read-out as part of a later pixel. Because of the asymmetry of this effect, a phase term is also present $[25,23]$ :

$$
\begin{aligned}
& M T F_{C T E}(f)=\exp \left[-n_{\phi} n_{\text {row }} \epsilon(1-\cos 2 \pi f L)\right], \text { and } \\
& P T F_{C T E}(f)=-n_{\phi} n_{\text {row }} \epsilon[2 \pi f L-\sin 2 \pi f L] \text {. }
\end{aligned}
$$

where $\epsilon$ is the charge transfer inefficiency. Modern CCDs are extremely efficient $\left(\epsilon<10^{-4}[23]\right)$ and the MTF loss is negligible.

\section{Temporal Characteristics of the Phosphor}

Temporal response characteristics of the CCD and other components can affect image quality. If light output from the phosphor persists longer than a clock cycle, then the light may be recorded in the next pixel. Maidment[21] proposed a simple model to account for its effect on image quality for a prototype scanning system. This model is similar to that proposed by others for correcting degraded images of moving objects for phosphor-based displays[26]. The rest of this work shall investigate this model in greater detail. In order to do this, several properties of phosphor luminescence must be understood. 


\subsection{Luminescence}

The process of $\mathrm{x}$-ray absorption and subsequent light emission from a phosphor is known as luminescence. A phosphor is generally a crystalline material that contains impurities or defect sites. These sites, or luminescent centres, are the location of the radiative recombination in which an electron in an excited state can return to a ground state by emitting its excess energy as a photon.

Luminescence is never an instantaneous process. This means that there is always some time, however small, between the absorption and emission stages. Luminescence emission will occur according to a probability distribution, $p(t)$. The duration of luminescence can be measured by a mean lifetime, $\tau$ :

$$
\tau=\int_{0}^{\infty} p(t) \cdot t d t
$$

The lifetime can vary from a few nanoseconds to several seconds depending on the phosphor. If the lifetime is relatively long, the phosphor is described as having persistence[27].

In the simplest form, luminescence involves an ensemble of independent two-level luminescence centres (atoms). Initially all the electrons are in the ground state (ignoring any thermal excitation). When $\mathrm{x}$-rays are absorbed in a material, photoelectrons and Compton recoil electrons are generated[28]. As these electrons slow down, they transfer their energy to other electrons in the crystal. If the electrons at a luminescence centre absorb this energy, they migrate to the excited state. At some time later, an electron returns to the ground state and releases its excess energy in the form of a light photon. Since each luminescence centre is independent, the probability of transition per unit time is a constant, $\alpha$. Hence, the rate of change of electrons in the excited state is:

$$
\frac{d n}{d t}=-\alpha n
$$

Solving this differential equation yields the number of electrons, $n$, remaining in the excited 
state:

$$
n(t)=n_{0} e^{-\alpha t}
$$

where $n_{0}$ is the number of electrons in the excited state at time, $t=0$. The number of photons released per unit time, or the intensity, will be equal to the rate of electron transitions:

$$
\begin{aligned}
I(t) & =-\frac{d n}{d t} \\
& =\alpha n_{0} e^{-\alpha t} \\
& =\frac{n_{0}}{\tau} e^{-t / \tau} \\
& =I_{0} e^{-t / \tau}
\end{aligned}
$$

where $\tau=\frac{1}{\alpha}$, is the luminescence lifetime of the phosphor, as defined in Equation 1.8, and $I_{0}=\frac{n_{0}}{\tau}$ is the light intensity at time, $t=0$.

If there is more than one pathway for the electron to follow (i.e. more than one excited state), or more than one type of luminescence centre, then each pathway may exhibit a different transition probability. Provided that these transitions remain independent, it is assumed that the intensity response is simply the sum of two or more weighted exponentials[29]:

$$
I(t)=I_{0} \frac{\sum_{j=1}^{N} \frac{w_{j}}{\tau_{j}} e^{-\frac{t}{\tau_{j}}}}{\sum_{j=1}^{N} \frac{w_{j}}{\tau_{j}}}
$$

where $w_{j}$ is the weighted contribution from the $j$ th component, $\tau_{j}$ is the corresponding lifetime, and $N$ is the number of different transition pathways. This equation is also useful as a model, without making a priori assumptions about the true functional form of the luminescence decay law $[29]$. Without knowledge of the luminescence decay, the number of components, $N$, is set to an arbitrary value, usually ranging from 2 to 15[29]. It should be noted that without prior knowledge of true form of the luminescence decay, the resultant parameters, $w_{j}$ and $\tau_{j}$, may not represent 
the true physical luminescence parameters; rather, they may simply represent empirical values that characterize the decay curve. For the purposes of characterizing the luminescence response in order to determine how it affects an imaging system, the true form is not required, and an empirical fit is sufficient.

In more complicated systems, there are deviations from the exponential model of luminescence[30, 31]. These generally involve dipole interactions between neighboring luminescence centres (which were previously assumed to be independent), charge transfer from the crystal lattice to the luminescence centre, or competitive radiationless transitions.

\subsubsection{Fluorescence, Phosphorescence, and Afterglow}

Luminescence is the most general term to describe the light emission phenomenon. Very often, more specific types of luminescence are defined to describe specific forms of their physical behaviour. Two common terms that are used are fluorescence and phosphorescence.

In the broadest sense, fluorescence is luminescence with very short lifetimes (from $10^{-9}$ to $\left.10^{-6} \mathrm{~s}\right)$ and phosphorescence has much longer lifetimes $\left(\approx 10^{-6}\right.$ and greater $)$. Both Guorescence and phosphorescence exhibit the exponential decay behaviour of Equation 1.14[32]. According to Blasse and Grabmaier[30], fluorescence and phosphorescence can be defined more accurately, based on whether the electronic transitions are "allowed" (fluorescence) or "forbidden" (phosphorescence). All electron transitions are governed by conservation laws of energy and momentum. If these laws are satisfied, the transition is allowed, and it occurs with a very high probability (short lifetime). At first glance, forbidden transitions seem to disobey these conservation laws and should never occur. However, various interactions between neighbouring electrons (within atoms and between atoms) ease the restrictions, and the so-called forbidden transitions actually occur. However, these transitions are at a greatly reduced probability, yielding the longer lifetimes of phosphorescence. The transitions for fluorescence and phosphorescence are shown in the energy diagram in Figure 1.8A-B.

The distinction between fluorescence and phosphorescence can be difficult to determine, es- 


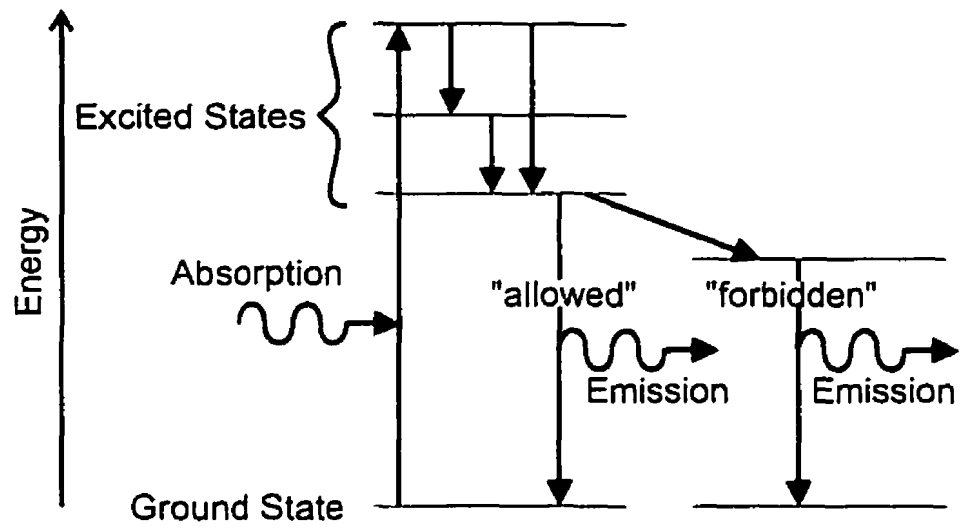

a)

b)

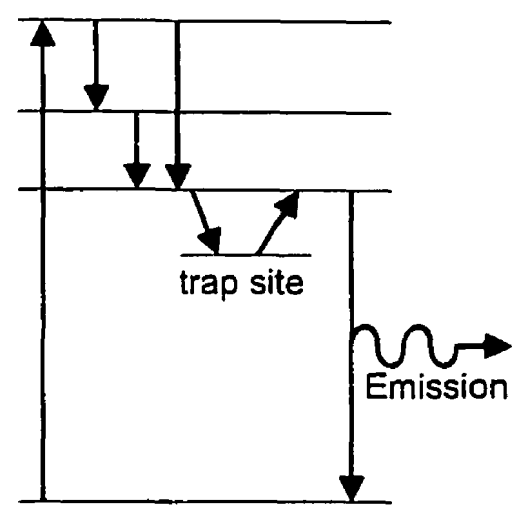

c)

Figure 1.8: An energy level diagram showing the distinction between a) fluorescence, b) phosphorescence, and c) afterglow. Fluorescence is a allowed transition, phosphorescence is a forbidden transition and afterglow is any form of emission following a localized trapping of the electron.

pecially when the lifetime is $\approx 10^{-6} \mathrm{~s}$. Even the "allowed verses forbidden" definition is difficult to employ. This is especially true in compounds with heavier atomic elements, where the interactions between neighbouring electrons are extremely complex. For this reason, the distinction of fluorescence and phosphorescence will not be used further in this work. Rather both of these forms will be referred to as primary luminescence.

There is another form of luminescence known as afterglow. Afterglow can continue long after the primary decay time of luminescence. It occurs because the radiative recombination of electrons and holes is delayed by the trapping of the electron (or hole) at a trap center. A simple illustration of afterglow is shown in Figure 1.8. Afterglow is strongly dependent on the defect concentration in the host lattices[33, 30]. Afterglow can often be lowered by the addition of special impurities to the phosphor.

The temporal response of afterglow tends to be complex, with several different mechanisms contributing to it $[32,31,30]$. Because of the relaxation of the trap states, the kinetics of afterglow often exhibit an inverse power law dependence with time[33]: 


$$
\Gamma(t)=\frac{I_{1}}{t^{\gamma}}
$$

where $\gamma$ is a constant usually $\leq 2$ and $I_{1}$ is the intensity at 1 second. This model is approximate, and valid only in the region $t \gg 0$. To eliminate the approach to infinity as $t \rightarrow 0$, a slightly better model has the form[32]:

$$
I(t)=\frac{I_{0}}{(1+b \cdot t)^{\gamma}}
$$

The constants $I_{0}, b$, and $\gamma$ are determined from experiment. There is some formal theory for the inverse power law model[31]. The theory works well for a subset of phosphors that are semiconductors which have been doped with acceptor impurities (electron traps) and donor impurities (hole traps). Luminescence occurs when a conduction electron can recombine with a hole at an donor site. Because of the band theory for semiconductors, a conduction electron can potentially recombine with a hole at any acceptor site in the crystal lattice. This means that at time, $t$, there are $n$ electron-hole pairs (EHPs) available, and any one electron can recombine with any of the $n$ available holes. Thus the rate of recombination for one electron is $\alpha \cdot n$. Since there are $n$ electrons available, then the recombination rate is the following:

$$
\frac{d n}{d t}=(\alpha \cdot n) \cdot n
$$

which, when solved for intensity is:

$$
I(t)=\frac{\alpha n_{0}^{2}}{\left(1+\alpha n_{0} t\right)^{2}}
$$

This is similar to the empirical Equation 1.17, with $I_{0}=\alpha n_{0}^{2}, b=\alpha n_{0}$ and $\gamma=2$. Because of spatial non-uniformities and electron scattering in the semiconductor, Equation 1.17 generally is a better model than Equation 1.19[32, 31]. 
It has been noted that afterglow is dependent on the host crystal, the presences of impurities, and by the concentration of defects which is, in turn, dependent on the processing techniques used[34].

In general, an $\mathrm{x}$-ray phosphor will exhibit both primary luminescence and afterglow:

$$
I(t)=I_{0}\left[\beta \frac{\sum_{i=1}^{N} \frac{w_{j}}{\tau_{j}} e^{-\frac{t}{r_{j}}}}{\sum_{i=1}^{N} \frac{w_{j}}{\tau_{j}}}+\frac{1-\beta}{(1+b \cdot t)^{\gamma}}\right]
$$

where $\beta$ is the relative contribution of primary luminescence to the total luminescence.

The temporal response of the phosphor can potentially lead to image quality degradation in a scanning system. As the detector scans, light emission continues from the phosphor, and the light adds signal to regions beyond the initial point of $x$-ray absorption. This will appear as a streak in the image, reducing resolution. If the phosphor has a particularly long response time or if the scan velocity is very high, then blurring due to phosphor persistence is expected to be very pronounced in the scan direction.

\subsection{Techniques for Measurement of Phosphor Luminescence Re- sponse}

In order to quantify the effect of persistence on image quality, the temporal response of the phosphor must be known. The measurement technique should allow the luminescence response to be measured under excitation conditions similar to those expected in radiographic use. The excitation source used for testing must be $\mathrm{x}$-rays, as the temporal response of the phosphor can vary slightly depending on the form of excitation[35][36] (e.g. electron beam, x-ray, or visible light). As well, to enable the measurement of the long term components in the response, a very long excitation pulse is desirable to enhance the relative intensity of these components. Selecting a measurement technique requires knowledge of the temporal range of interest. In the scanning system, the distance, $\Delta d$, over which the blur occurs is expected to be proportional to the product of the scan velocity, $v$, 
and the phosphor lifetime, $\tau$ :

$$
\Delta d \approx v \tau
$$

Assuming that blurring of $10 \mu \mathrm{m}$ or more may be significant, and that practical scanning velocities are probably no more than $200 \mathrm{~mm} / \mathrm{s}$, then the lifetime temporal range of interest is approximately $50 \mu \mathrm{s}$ or more.

There are several techniques for measuring the luminescence response. Perhaps the most accurate is the time-correlated single photon counting (SPC) method[29]. A block diagram of an SPC experiment is shown in Figure 1.9. A trigger signal is sent to the excitation source (usually a laser) which emits a very short pulse of radiation. The trigger pulse is also sent to the start input of the time-to-amplitude converter (TAC). The phosphor luminesces in response to the excitation radiation. At some time later, dictated by the transition probability, the photomultiplier detects the first luminescence photon, which sends a stop signal to the TAC. The TAC outputs a signal that is proportional to the time between the start and stop pulse. This is recorded as a single count by a multichannel analyzer. These counts are plotted as a function of time. This process is repeated continually, until the counts are sufficient to produce an accurately plotted decay curve.

The SPC method is probably the most common method for measuring luminescence because it can achieve very high temporal resolution $(\approx 20 \mathrm{ps})[29]$, and it can measure very short decay times. Because this is a counting experiment, the noise is governed by Poisson statistics[36]. However, the SPC method is generally limited to short counting times and low intensities. Monitoring very slow responses ( $1 \mathrm{~ms}$ to 1 second) would require an extremely long experiment (at low intensities) or, if high intensities are used, the possibility of pulse pile-up can occur[29, 36]. Pile-up occurs when more than one photon is detected during a single time interval for each excitation; and leads to distortion in the measured temporal response.

An alternative luminescence measurement technique is the modulation method, shown in Figure 1.10. In this technique, the excitation source is modulated sinusoidally at frequency, $f$, 


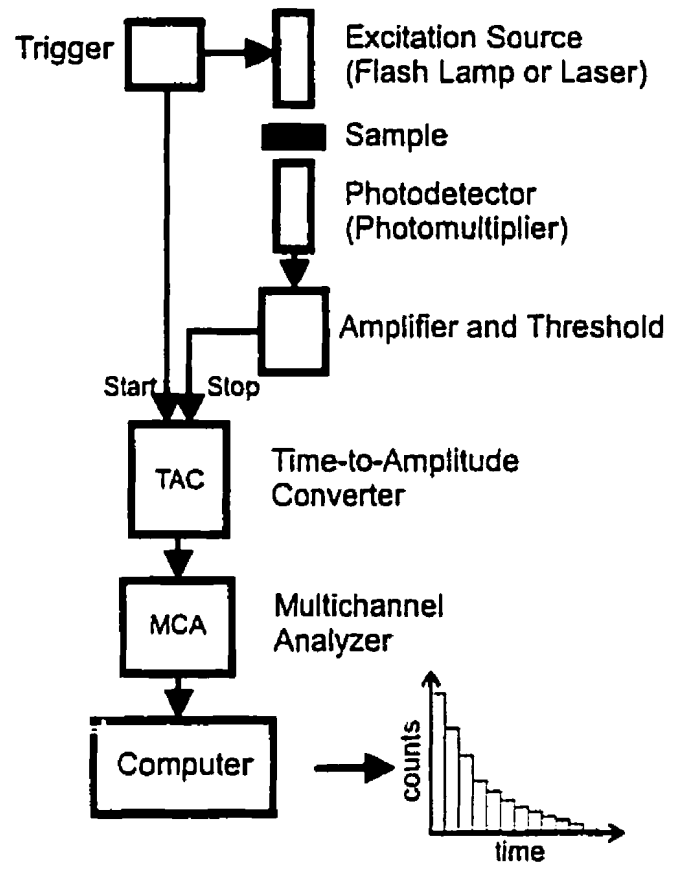

Figure 1.9: Schematic of a single photon counting (SPC) luminescence apparatus.

and the phase delay will yield the luminescence lifetime. Assuming the phosphor exhibits an exponential decay as in Equation 1.14, then the phase delay, $\theta$, between the excitation sinusoid and the luminescence response sinusoid will be:

$$
\tan \theta=2 \pi f \tau
$$

This method can be used to measure a range of luminescence lifetimes (as low as $200 \mathrm{ps}[29]$ to as long as the excitation source is stable). Unfortunately this method is only useful if the sample is known to have a single exponential component. More than one component (or a non-exponential response) can be determined, but a model of the response must be known a priori in order for the modulation method to be useful.

A third method is known as the pulse sampling technique, shown in Figure 1.11. This method uses an excitation pulse of radiation that hits the phosphor, and a photodetector that collects the luminescence radiation. A sampling oscilloscope is used to monitor the photocurrent 


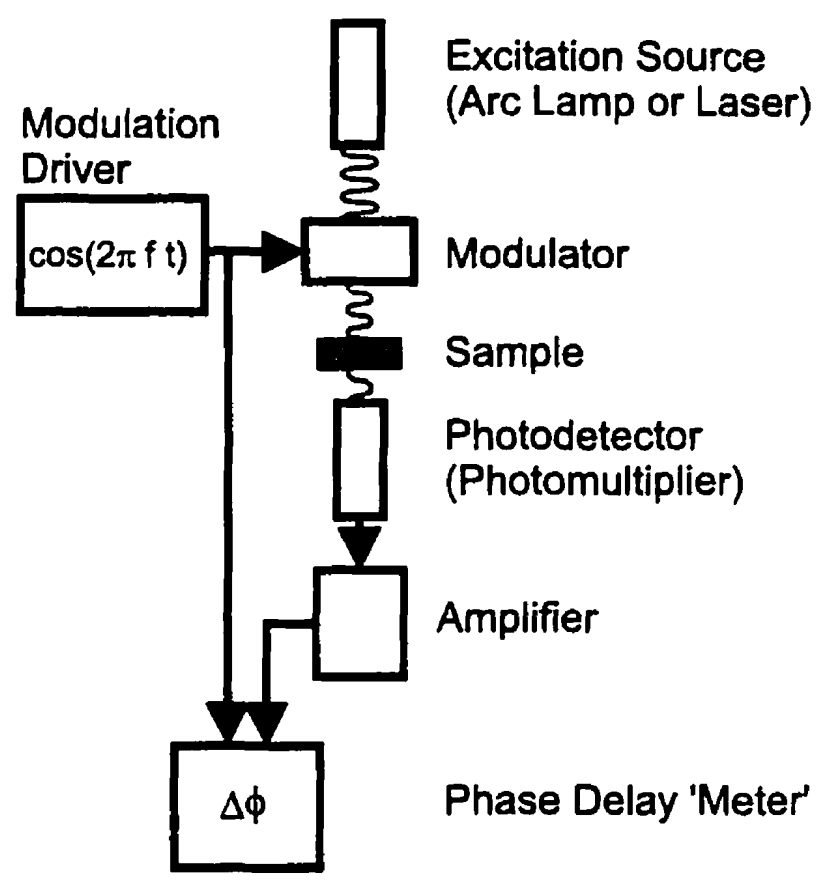

Figure 1.10: Schematic of the modulation method apparatus for measuring luminescence response generated. This method suffers from more noise sources than the SPC technique, including gain variation of the detector and electronic noise. The time resolution is also significantly reduced compared to the SPC method.

However, if very high temporal resolution is not required, the pulse sampling technique can be a more flexible method. It can be used for any intensity of excitation radiation, and it can be used to monitor response over very long periods of time. As well, the excitation pulse can be of an arbitrary shape and width. Unlike the SPC method, the luminescence signal can be monitored well beyond the first photon event. The pulse sampling technique was used for the measurements in Chapter II, because of the increased flexibility with respect to the time range of interest $(\approx I \mu \mathrm{s}$ to $1 \mathrm{~s})$ and the ability to use higher intensity excitation. 


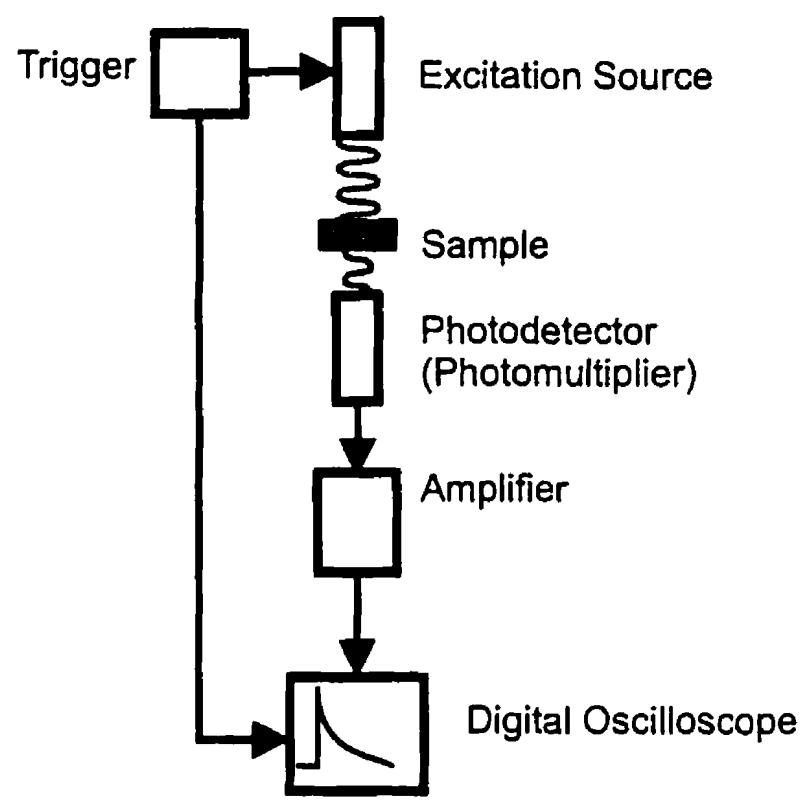

Figure 1.11: Schematic of the pulse sampling method apparatus for measuring luminescence response

\subsection{Summary and Outline of Thesis}

There is a potential for digital radiography to surpass conventional film-screen radiography. Because of a relatively low cost and use of light detection technology, the phosphor coupled digital $\mathrm{x}$ ray system is ready for clinical use. These systems are ready to be tested to determine if the improvements of increased dynamic range, computer image enhancement, and computer aided diagnosis will actually lead to more sensitive and more accurate disease detection.

One design of a phosphor-coupled system is a slot scanning system that uses a light sensitive CCD in TDI mode. In order for the slot-scanning system to perform, every stage of the system must be optimized to maintain the highest possible image quality. The scanning motion can introduce some loss of resolution into the final image. Earlier work by Maidment has demonstrated that the temporal response of the phosphor may be responsible for some of this image degradation.

The following chapter will define and quantify the effect of the temporal response of phosphors on the resolution of a slot scanned TDI system. By comparing the response of several 
commercially available phosphors, the relative significance of this effect will be determined. Both the short primary luminescence and long-term afterglow will be examined.

Future work, Chapter III, will examine the selection of an ideal phosphor for a digital scanning system. The relative importance of using the complete optical transfer function as opposed to only using the modulation transfer function as a measure of image quality will be analyzed. Finally, the future role of direct conversion detectors as an alternative to phosphor coupled systems will be discussed. 


\section{Chapter 2}

\section{Effect of Phosphor Persistence on}

\section{Resolution in X-ray Scanning Systems}

\subsection{Introduction}

As described in Chapter I. phosphor-based slot scanning systems offer many advantages over conventional film-screen systems. However, the temporal response associated with the image acquisition can potentially reduce resolution in the scan direction. One aspect of the temporal response of the detector is the luminescence characteristics of the phosphor. As mentioned previously, the temporal response of a phosphor is sometimes referred to as persistence. Earlier work by Maidment[21] has suggested that loss in resolution due to phosphor persistence may be relatively large. If the temporal response of the phosphors is known, an ideal phosphor can be selected for the scanning system that will introduce minimal blurring due to persistence. Therefore, it is necessary to accurately determine the phosphor response and quantitatively describe how persistence will reduce resolution in scanning system.

The following sections describe how the temporal response of phosphors affects image quality based on a simple model for the scanning technique. In order to measure the temporal response of 
several commercial phosphors, a pulse sampling method was used. Both the primary luminescence and afterglow of the phosphors were measured. From the luminescence response curves, the effect on spatial resolution was estimated for a scanning $x$-ray system.

\subsection{Background and Theory}

\subsubsection{Luminescence in a Scanning System}

Figure 2.1 shows the simplified operation of the CCD/phosphor assembly. Consider the example of imaging an object that is composed of a narrow slit-i.e. opaque to $x$-rays except in a narrow localized region. During the scan, an $x$-ray beam moves in synchrony with the detector. The $\mathrm{x}$-rays that pass through the slit are absorbed in the phosphor which emits light. The light, in turn, generates EHPs in the potential wells (the potential barriers are not shown in Figure 2.1, refer to Figure 1.7 for a more complete picture of charge localization and transfer). The charge is transferred along the CCD column by a series of clock pulses as described in the previous chapter. As the detector assembly moves, a new region of the phosphor absorbs the $\mathrm{x}$-rays and begins to emit light. If the phosphor has a sufficiently slow temporal response, then light emission continues from the phosphor even after passing beyond the slit, as shown in Figure 2.1B. Adjacent CCD wells are filled with charge, producing an image of the slit that is blurred out over several pixels.

Since the charge packets remain stationary with respect to the object (ignoring the jitter motion caused by the clock-pulsed charge transfer), and the detector moves at a velocity, $v$, with respect to the object, then it follows that the phosphor must appear to move at $v$ with respect to the charge packets (and hence the image). If the phosphor absorbs a burst of $x$-rays at position, $x=0$, and assuming that the luminescence response follows Equation 1.15, the resultant image will be an exponentially decaying blur (ignoring any afterglow): 


\section{A) Ideal TDI}
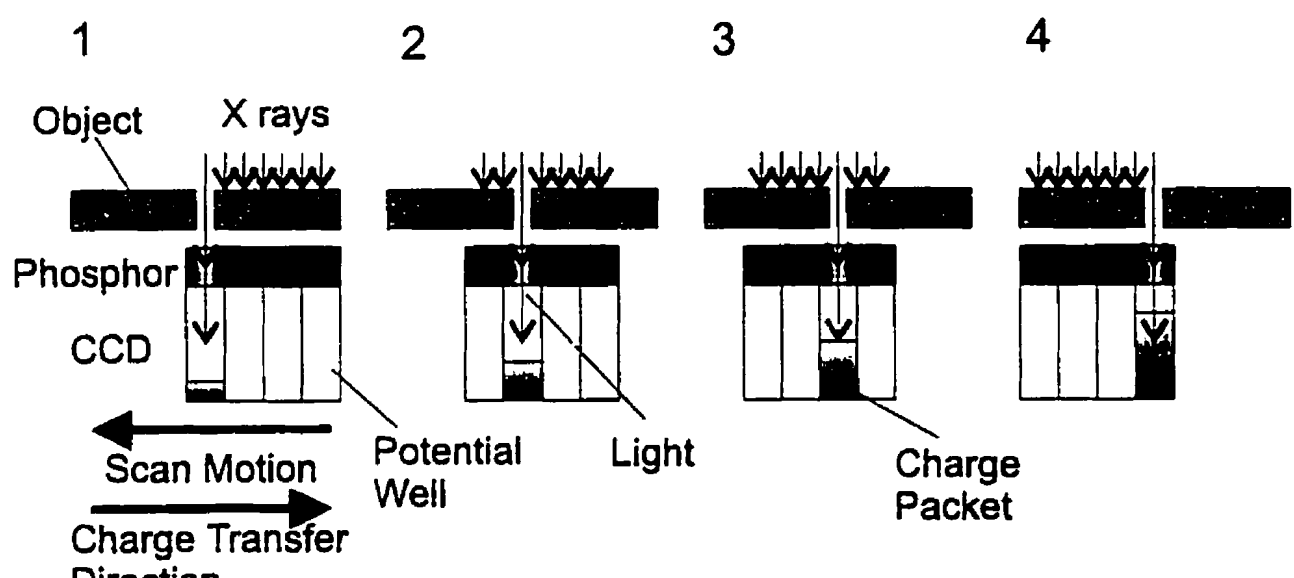

Digital Image

Direction

\section{B) TDI with Phosphor Persistence}

Digital Image
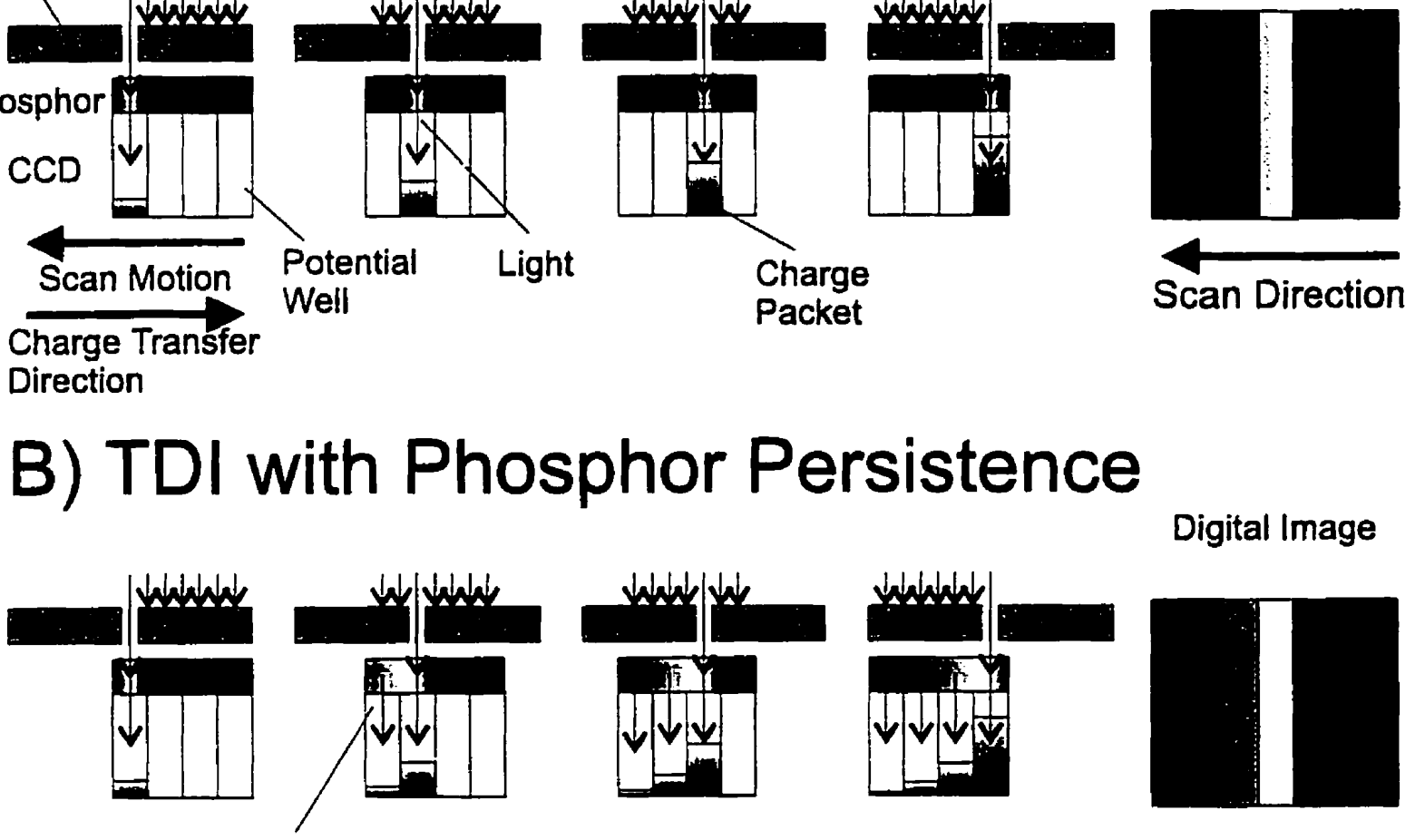

Prolonged

Light Emission

Figure 2.1: Simplified model of TDI charge transfer while scanning an x-ray slit, shown in crosssection through one of the CCD columns. In A) the phosphor has an ideal instantaneous luminescence, and $B$ ) shows TDI charge transfer with a phosphor that has persistence. 


$$
I(x)= \begin{cases}0 & \text { if } x<0, \\ I_{0} \frac{\sum_{j=1}^{N} \frac{w_{j}}{\tau_{j}} e^{-\frac{x}{v \cdot \tau_{j}}}}{\sum_{j=1}^{N} \frac{w_{j}}{\tau_{j}}} & \text { otherwise } .\end{cases}
$$

where $I_{0}$ is the intensity at time, $t=0$, and $N$ is the number of primary luminescence components. For each exponential component, $w_{j}$ is the relative weighting, $\tau_{j}$ is the luminescence lifetime. Ignoring all other blurring sources, Equation 2.1 describes a "one-sided" point-spread function (PSF). Using linear system theory, the one dimensional optical transfer function (OTF) is the Fourier transform of Equation 2.1. The OTF for phosphor persistence is $O T F_{p}(f)$ :

$$
O T F_{p}(f)=\sum_{j=1}^{N} \frac{w_{j} v}{1+2 \pi i \tau_{j} v f} .
$$

Similarly, an equation for the PSF induced by afterglow can be modeled:

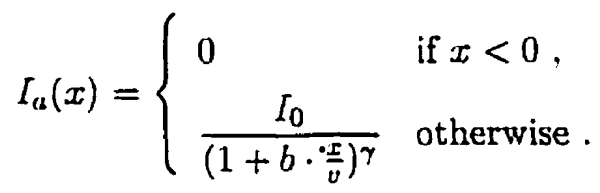

As mentioned in Chapter I, afterglow can be non-linearly dependent on intensity and pulse duration[33]. Because of this, linear systems theory cannot strictly be used (i.e. the OTF cannot be properly defined). However, if the afterglow is measured at a high intensity, following a reasonably long pulse, this could be considered to be the worst case or at the very least a representative case of afterglow response. By calculating the OTF corresponding to this afterglow curve, this would place an upper limit on the resolution loss due to afterglow. Unfortunately, there appears to be no analytic Fourier transform of Equation 2.3. Instead, the OTF can be approximated numerically using a fast Fourier transform technique:

$$
O T F_{a}(f)=\operatorname{FFT}\left\{I_{a}(x)\right\}
$$


As mentioned, in Chapter I, the MTF is often used as the sole description of blurring in an imaging system. However, with the asymmetry involved in the scanning process, the complete OTF must be considered. From Equation 2.2, for example, the OTF can be separated into the modulation transfer function and the phase transfer function. For $N=1$, the MTF and PTF will be:

$$
\begin{gathered}
M T F_{p}(f)=\frac{1}{\sqrt{1+(2 \pi i \tau v f)^{2}}}, \text { and } \\
\operatorname{PTF}_{p}(f)=\arctan (-2 \pi \tau v f) .
\end{gathered}
$$

Because the OTF has a non-linear phase component, the resultant image can be quite different than if the magnitude were considered alone. Shown in Figure 2.2 are differences between a PSF produced from considering the MTF alone, verses the PSF corresponding to the complete OTF (both phase and magnitude). The plots are shown for a lifetime of $\tau=1 \mathrm{~ms}$ and velocities of $v$ equal to 40 and $80 \mathrm{~mm} / \mathrm{s}$.

From Figure 2.2, notice that the distortion induced by the phase transfer function actually increased the full-width half maximum by 3.5 to 8 times. It is clear that the MTF is not sufficient for describing the image quality loss induced by the temporal response of the phosphor, rather the complete OTF must be used.

\subsubsection{Curve Fitting of the Luminescence Decay}

In principle, the measurement of luminescence response could be done by irradiating the sample by an impulse of exciting radiation, $\delta(t)$, and monitoring the intensity of the emitted light as a function of time. The resultant curve could be fit to Equation 1.15 (ignoring afterglow). However it is often difficuit or impossible to deliver a sharp pulse of $x$-rays. If the pulse has some non-ideal 


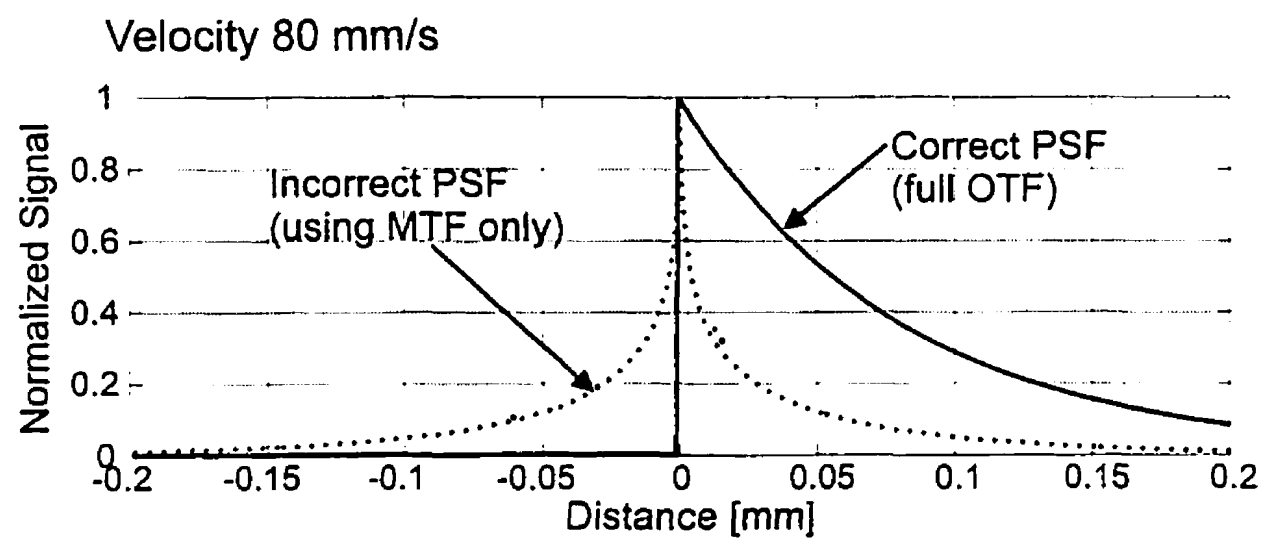

Velocity $40 \mathrm{~mm} / \mathrm{s}$

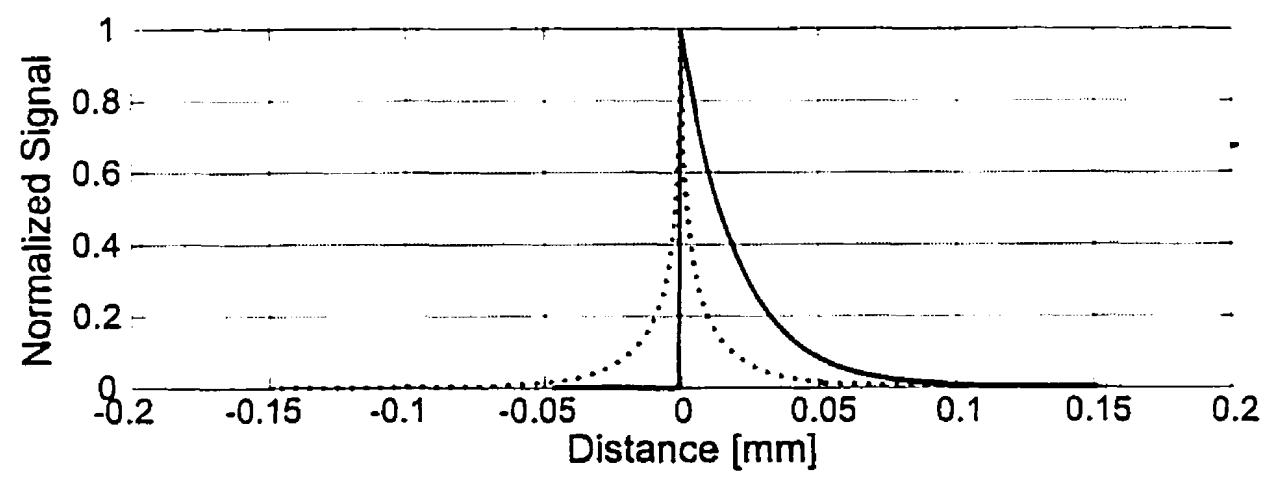

Figure 2.2: Effect of the phase component on the point spread function. For a phosphor with a $\tau=1 \mathrm{~ms}$ and scanning velocities of $v=80 \mathrm{~mm} / \mathrm{s}$ (top graph) and $v=20 \mathrm{~mm} / \mathrm{s}$, the graphs show the true PSF for phosphor persistence which is characterized by a complex OTF. If only the MTF was used for characterization, a different (incorrect) PSF is determined. 
shape, $P(t)$, and the phosphor has an impulse response, $I(t)$ then the resultant curve, $R(t)$, will be:

$$
R(t)=\int_{0}^{t} P(t-s) I(s) d s=P(t) * I(t)
$$

If $P(t)$ has a very sharp fall time (compared to the shortest lifetime component of the phosphor), then the tail portion of $R(t)$ would be approximately equal to $[(t)$, and Equation 1.15 could be fit directly to it. However, if $P(t)$ has a fall time that is on the order of the lifetime of the phosphor, then a fit of Equation 1.15 to $R(t)$ would yield overestimates of the lifetimes $\left(\tau_{j}\right)$. Thus, the influence of the $x$-ray pulse shape must be included in the determination of the lifetime components.

If $R(t)$ and $P(t)$ are known, then $I(t)$ could be calculated by performing a deconvolution (the inverse of Equation 2.7). However, deconvolutions are prone to large errors and often $I(t)$ cannot be recovered[37]. However, Grinvald and Steinberg[38] have described a least squares technique for obtaining $I(t)$, known as iterative deconvolution. This technique calculates the deconvolution indirectly, avoiding the errors associated with direct deconvolution. Using iterative deconvolution, any arbitrary pulse shape can be employed for the exciting radiation.

Iterative deconvolution is an optimization process in which both the true $\mathrm{x}$-ray pulse shape, $P(t)$ and the resultant response, $R(t)$, are recorded. The known $x$-ray pulse shape is then convolved with an estimated phosphor luminescence model, such as Equation 1.15. This produces an estimate of the overall response, $\hat{R}(t)$. By comparing $\hat{R}(t)$ and $R(t)$ the parameters for Equation $1.1 \bar{s}$ can be updated by using the weighted non-linear least squares technique, in which the weight sum of squared differences, $\chi^{2}$ is minimized:

$$
\chi^{2}=\sum_{i=1}^{m} \frac{1}{\sigma_{i}^{2}}\left(R_{i}-\hat{R}_{i}\right)^{2}
$$

where $m$ is the number of data points, $\left\{R_{i}\right\}$ is the set of data measurements of $R(t)$ and $\sigma_{i}$ is the experimental uncertainty of each data point. In this work, the least square method was a non-linear 
least squares method based on the Levenberg-Marquhardt technique. A complete description of this least squares method is supplied in Appendix B. The parameters $\left(w_{j}\right.$ and $\tau_{j}$ ) are updated according to this non-linear least squares technique, and a new $\left\{\hat{R}_{i}\right\}$ is recalculated and a new $\chi^{2}$ is determined. New parameters are obtained and this cycle is repeated until $\chi^{2}$ reaches a minimum. An important measure of "goodness of fit" is the reduced chi-square, $\chi_{R}^{2}$, which is defined as:

$$
\chi_{R}^{2}=\frac{\chi^{2}}{m}
$$

For luminescence measurements, $\chi_{R}^{2}$ should fall between 0.8 and 1.2 , although fits may be deemed to be acceptable with $\chi_{R}^{2}$ as great as 1.5[29]. In addition, the weighted residuals should be examined for any obvious trends, indicating an incorrect fit[38].

When using the sum of exponentials as the luminescence model (Equation 1.15), there is a high degree of correlation between the parameters (i.e. a change in one parameter can be compensated by changes in other parameters without significantly affecting the overall curve shape). Grinvald and Steinberg [38] suggested that the confidence regions of the fitted parameters should be determined to obtain an estimate of the uncertainty associated with the terms. When the leastsquares regression is performed, a set of estimates of the parameters, $\left\{w_{1}^{0} w_{2}^{0} \ldots, \tau_{1}^{0} \tau_{2}^{0} \ldots\right\}$ is obtained. These estimates yield a minimum chi-squared value, $\chi_{0}^{2}$. Any other values for the parameters yields chi-square greater than the minimum, $\chi^{2}>\chi_{0}^{2}$. A confidence region can be set by an ellipsoidal region around the minimum, such that:

$$
\chi^{2}=\chi_{0}^{2}\left[1+\frac{N}{m-N} F(N, m-N, p)\right]
$$

where $F(a, b, p)$ is the inverse F-distribution function[39], $N$ is the number of parameters, $m$ is the number of points, and $(p \times 100 \%)$ is the desired confidence level. Equation 2.10 is only exact for the linear case, but is approximately true for non-linear models[40]. To find the parameters that correspond to the confidence ellipsoid, various values are tried for one parameter (e.g. $w_{1}>w_{1}^{0}$ ) 
and the least-square routine is repeated, keeping that parameter fixed. When the least-square routine yields the $\chi^{2}$ set by Equation 2.10, this defines the upper limit for that parameter $\left(w_{1}\right)$. This is repeated for every parameter and for the lower confidence limits as well. This technique of uncertainty estimation is advantageous because it makes no assumptions about independence of the parameters.

\subsubsection{Afterglow}

When measuring afterglow (i.e. long phosphor responses), the fit can be performed over a region relatively far from the $x$-ray cut-off $(>100 \mu \mathrm{s})$. Because of this, it can be assumed that the $x$-ray pulse is approximately a square pulse. To simplify the fitting algorithm, the convolution was done analytically:

$$
\begin{aligned}
R_{a}(t) & =P(t) * I(t) \\
& =\Pi\left(\frac{t-\frac{T}{2}}{T}\right) * \frac{l_{0}}{(1+b \cdot t)^{\gamma}} \\
& = \begin{cases}\frac{I_{0}}{b(\gamma-1)}\left[1-\frac{1}{(1+b \cdot t)^{\gamma-T}}\right] & \text { if } t>T \\
\frac{I_{0}}{b(\gamma-1)}\left[\frac{1}{(1+b(t-T))^{\gamma-T}}-\frac{1}{(1+b \cdot t)^{\gamma-T}}\right] & \text { otherwise }\end{cases}
\end{aligned}
$$

where $\Pi\left(\frac{t}{T}\right)$ is the rect function which is defined as:

$$
\Pi\left(\frac{t}{T}\right)= \begin{cases}\frac{1}{T} & \text { if }|t|<\frac{T}{2} . \\ 0 & \text { otherwise. }\end{cases}
$$

Equation 2.11 was fit directly to the tail portion of the data as shown in the sketch in Figure 2.3. By extrapolating back to the time of $\mathrm{x}$-ray shutoff, $t=T$, the relative contribution of the afterglow to the primary luminescence can be determined. If $R_{T}(t)$ is the total luminescence response, and $R_{a}(t)$ is the afterglow, then the ratio of primary luminescence to the total luminescence is:

$$
\beta \approx \frac{R_{T}(T)-R_{a}(T)}{R_{T}(T)}
$$


Combining Equation 2.2 and Equation 2.4 and knowing the relative intensity contribution, $\beta$, the total OTF due to phosphor persistence can be calculated for a slot scanning system:

$$
O T F_{T}(f)=(1-\beta) \mathrm{FFT}\left\{\frac{1}{(1+b \cdot x / v)^{\gamma}}\right\}+\beta \sum_{j=1}^{N} \frac{w_{j} v}{1+2 \pi i \tau_{j} v f}
$$
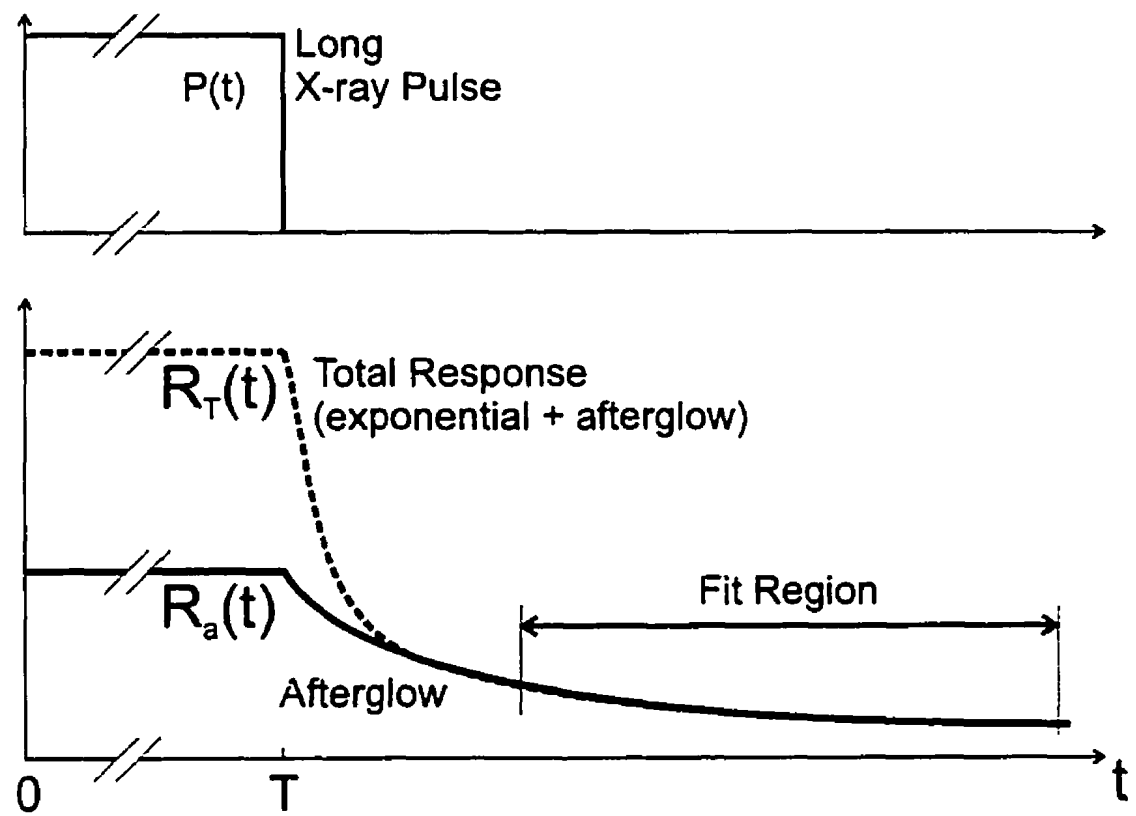

Figure 2.3: Sketch of the phosphor response to a very long $x$-ray pulse. The solid line is the response due to afterglow alone and the dotted line is the complete phosphor response. Note that the $x$-ray pulse has been assumed to be a square pulse

\subsection{Method}

A schematic of the pulse sampling luminescence measurement apparatus is shown in Figure 2.4. The detectors consisted of two photomultipliers (Hamamatsu Model R259). One PMT was mounted with a bismuth germanate (BGO) scintillator ( $3 \mathrm{~mm}$ thick), used to measure the $\mathrm{x}$-ray pulse directly. This scintillator is known to have a fast primary luminescence, a biexponential with lifetimes of $\tau_{1}=60 \mathrm{~ns}$ and $\tau_{2}=300 \mathrm{~ns}$ and demonstrated to have very little afterglow[36]. The phosphor material to be tested was mounted in the entrance port of the light sphere. The second PMT was 
placed on a second port of the light sphere, outside of the x-ray beam. A baffle was used inside sphere to prevent a direct path for the light photons emitted from the phosphor to reach the PMT.

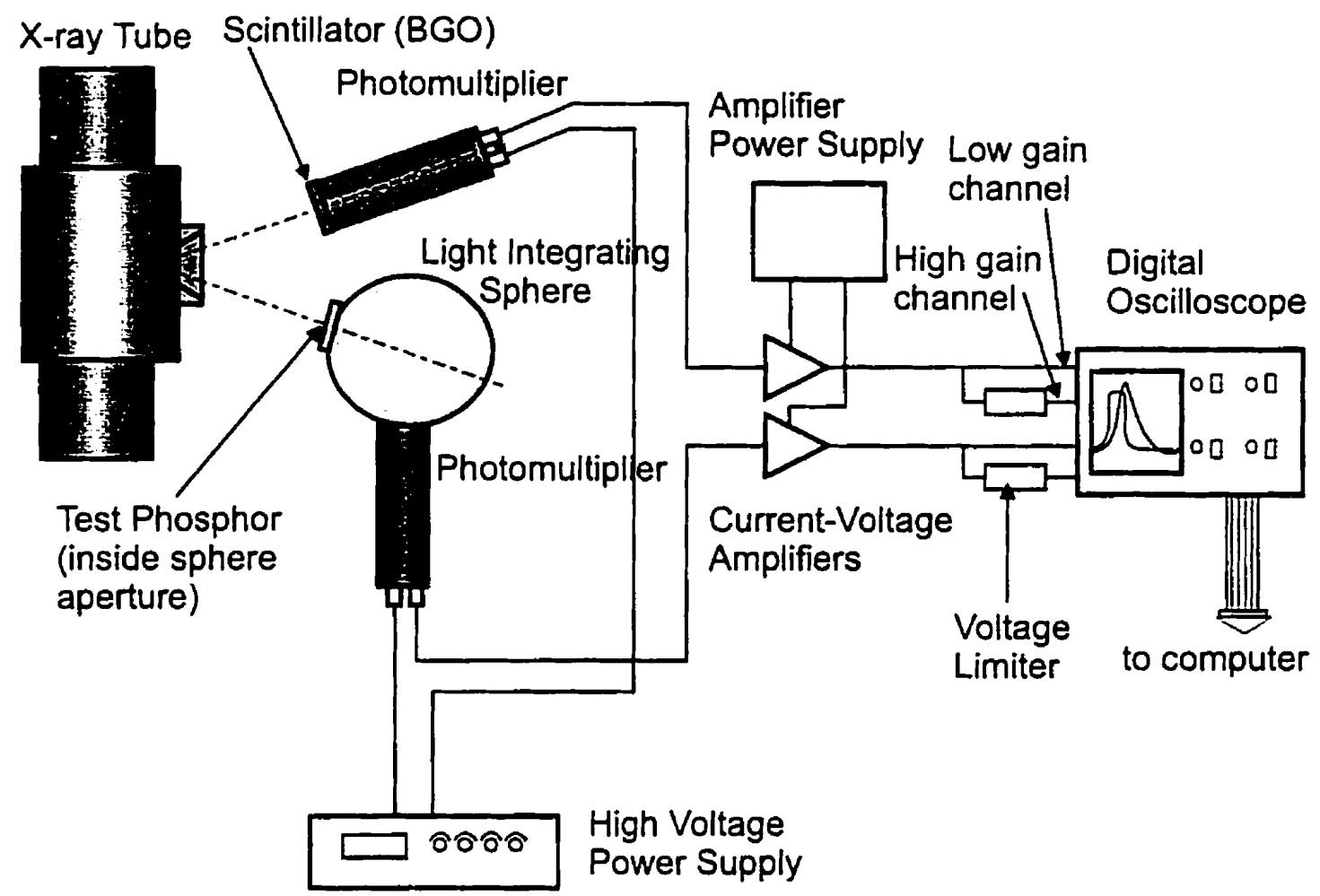

Figure 2.4: A schematic of the pulse sampling experiment

The $\mathrm{x}$-rays were generated by a Machlett rotating tungsten anode $\mathrm{x}$-ray tube powered by a GX1050 high voltage generator. The pulsing of the $x$-rays was achieved by a computer controlled high frequency regulator (Edec $100 \mathrm{Z}$ ). The tube was operated at $28 \mathrm{kVp}$ and $100 \mathrm{~mA}$ (nominal). The signal from the PMTs were sent through a current-voltage pre-amplifier with a gain of $2.85 \times 10^{5}$ V/A. The signals were collected by a digital oscilloscope (Lecroy $9314 \mathrm{~A}$ ).

The oscilloscope had 8 bit digitization, and so the signal from the phosphor-PMT was split into two channels with low (16-64 bits/V) and high (2130 bits/V) digital gains, in order to increase the dynamic range. A Schottky diode was used to prevent the signal from saturating the high gain channel. 
To reduce noise, 50 successive pulses were averaged together. By stitching the traces from the two channels together for the phosphor signal, a piece-wise dynamic range of 16-17 bits (yielding at least 4 orders of magnitude of sensitivity) was obtained.

Short $\mathrm{x}$-ray pulses $(500 \mu \mathrm{s})$ were used to produce phosphor responses that were dominated by primary luminescence. The traces were digitized at $1 \mu \mathrm{s}$ intervals for a duration of $7 \mathrm{~ms}$. Each pulse was separated by a 3 second settling time (to reduce afterglow influence from previous pulses), but to improve measurement stability, the filament current and rotor were kept at a constant level for a total acquisition time of 150 seconds.

Since a pulse sampling technique was used, the variance on each point expected to be the quadrature sum of the Poisson noise, digitization error, and background electronic noise.

$$
\sigma_{i}^{2}=\left(c e \sigma_{q u a n t u m}\right)^{2}+\sigma_{d i g}^{2}+\sigma_{b k g d}^{2}
$$

where $c=2.85 \times 10^{5} \mathrm{~V} \cdot \mathrm{A}^{-1}$ is gain of the current-voltage amplifier, $e$ is the charge on an electron. The digitization error was assumed to be $\sigma_{d i g}=\Delta / \sqrt{12}$ [41] where $\Delta$ is the step-size of the least significant bit $\left(8\right.$ bits). By averaging, $\sigma_{\text {dig }}$ was reduced further by a factor of $\sqrt{N_{a v g}}$. The background noise was calculated by acquiring a trace and calculating the standard deviation for no $\mathrm{x}$-rays, without phosphor present and was measured to be $100 \mu \mathrm{V}$.

The light photon noise, $\sigma_{\text {quantum }}$ (in electrons/s) can be estimated assuming quantum noise analysis:

$$
\sigma_{q u a n t u m}^{2}=\frac{1}{N_{a v g} \cdot T} \eta_{p} \cdot \phi_{i}\left(g^{2}+\sigma_{g}^{2}\right)
$$

where $N_{a v g}$ is the number of traces averaged together, $T$ is the integration time of the amplifier $(1.5 \mu \mathrm{s}), \eta_{p}$ is the quantum efficiency of the photocathode $(12 \%$ at $550 \mathrm{~nm}), \phi$ is the photon flux (photons/s), and $g$ is the electron multiplication factor for the PMT. The multiplication factor was determined by comparing the output for the PMT for a stable green LED to the output of 
a calibrated light meter, and was measured to be $6.1 \times 10^{5}$ electrons/photon. The variance in the PMT gain, $\sigma_{g}$, was assumed to be Poisson, hence $\sigma_{g}^{2}=g[36]$.

The variation in the number of $\mathrm{x}$-rays per sample point during the $\mathrm{x}$-ray pulse would also be a source of error. The maximum x-ray exposure rate was $184.5 \mathrm{mR} \cdot \mathrm{s}^{-1}$ at the surface of the phosphor, measured with an ion chamber placed at the same position as the port of the light sphere. Based on the conversion relation between exposure and photon number from Johns and Cunningham[28], this corresponds to approximately $1000 \mathrm{x}$-ray photons $/ \mu \mathrm{s} / \mathrm{cm}^{2}$ incident on the phosphor. Assuming Poisson statistics apply, this yields a relative noise of approximately $\pm 3 \%$ per point. However, the $\chi^{2}$ sum was limited to the region of the pulse following $\mathrm{x}$-ray shut-off, avoiding this source of noise. The variation in the $x$-ray signal will also affect the initial conditions of the pulse, especially the initial intensity, $I_{0}$. However, the coefficients of interest are the lifetimes, $\tau_{j}$. and the relative weights, $w_{j}$. Variations in $I_{0}$ affect all regions of the pulse equally and are of little interest. Thus, the $\mathrm{x}$-ray variations were assumed to have a minor contribution to the weighting terms, $\sigma_{i}$. In order to confirm this, the noise determined from Equation 2.15 and the noise estimated by calculating the point-to-point standard deviation of Give repeated experiments for $\mathrm{Gd}_{2} \mathrm{O}_{2} \mathrm{~S}$ : Tb is shown in Figure 2.5. Note the discontinuity in the uncertainty produced by the splicing of the high and low gain channels. The agreement between the curve and the data suggests that, to a first order approximation, the variation in the x-ray signal can be ignored.

In order to investigate much longer components (i.e. afterglow), a very long excitation pulse was used. The convolution of a longer $x$-ray pulse with the phosphor response, will tend to enhance the contribution of the longer components relative to the short components. Because of the larger signal, these components will be more accurately measured than if investigated with short pulse excitation.

The long pulse or step response of the phosphors was measured using a 500 ms x-ray pulse. Again, fifty pulses were acquired to reduce noise. The sampling interval was increased to $20 \mu \mathrm{s}$ and a $0.5 \mathrm{~s}$ trace was collected. There was approximately 10 seconds of settling time between pulses. 

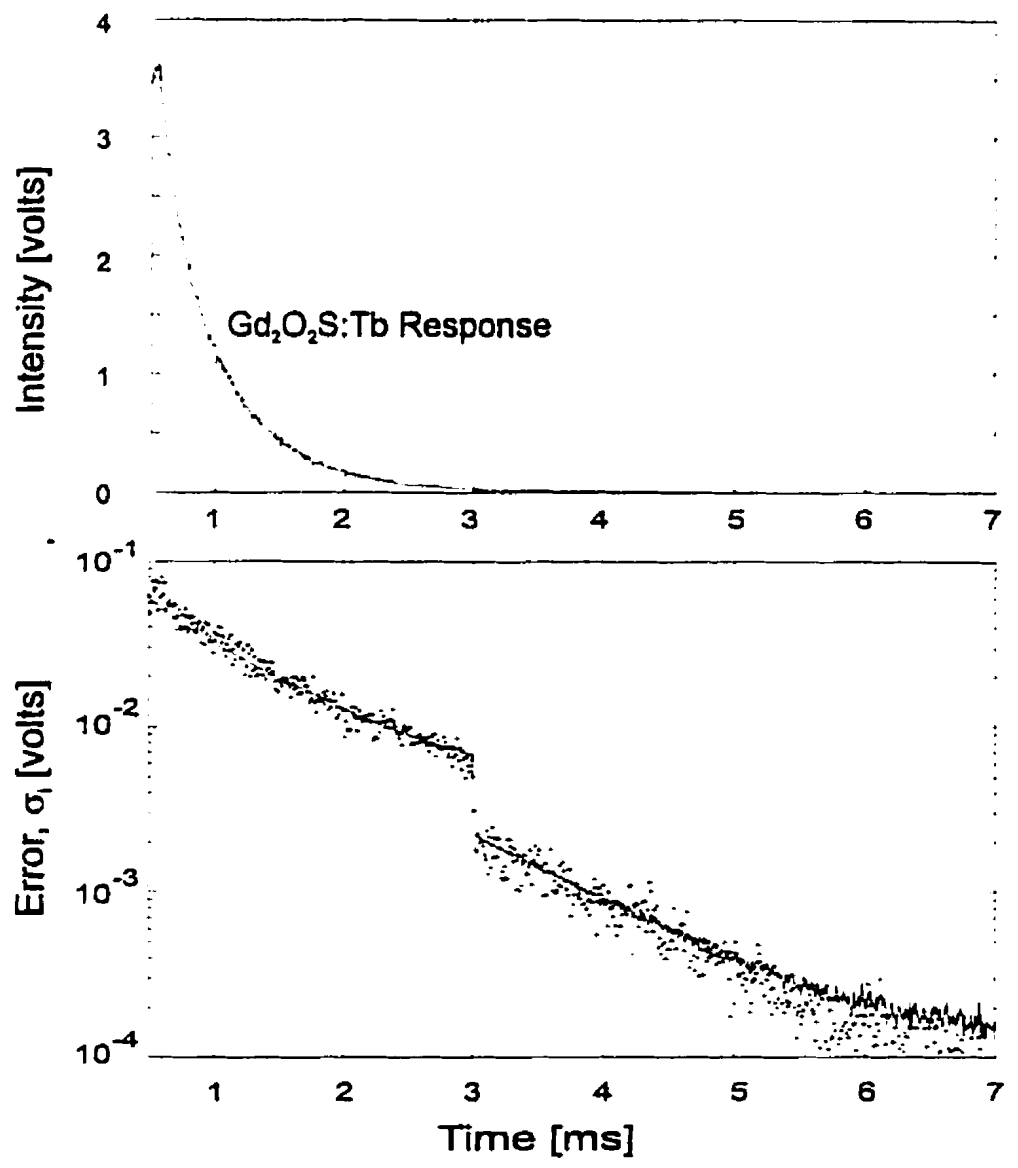

Figure 2.5: An example of the pulse response of $\mathrm{Gd}_{2} \mathrm{O}_{2} \mathrm{~S}: \mathrm{Tb}$ (top graph - linear plot) and the corresponding point-by-point error estimate (bottom graph - semilog plot). In the bottom graph, the solid line is based on noise predicted by Equation 2.15 and the points are the standard deviations of 5 repeated experiments. For clarity, only every 10th data point is shown.

Prior to each pulse, the filament was heated for 2 seconds to reduce temperature variations from pulse to pulse.

\subsection{Results}

An example of the short pulse response data is shown in Figure 2.6 comparing the $x$-ray pulse measured by the BGO crystal and the response of $\mathrm{Gd}_{2} \mathrm{O}_{2} \mathrm{~S}$ :Tb. Eight phosphors were tested. Table 2.1 lists the phosphors tested, the dominant luminescent chemical, the relative steady-state 
light output, the dominant wavelength of light output and the quantum efficiency of the phosphors for the $28 \mathrm{kVp}$ spectrum. The relative light intensity of each phosphor was measured during a 500 ms x-ray pulse with the apparatus shown in Figure 2.4. Note that the relative light output has not been corrected for the spectral response of the PMT, which has a relatively flat response between 300 and $500 \mathrm{~nm}$. Most of the phosphors had blue or green emission and thus the correction should be minor. Only the light output for $\mathrm{YTaO}_{4}$ and $\mathrm{CsI}: \mathrm{Tl}$ may be underestimated because $\mathrm{YTaO}_{4}$ has emission that extends to the u-v and CsI:Tl has emission that extends towards the red end of the spectrum. The quantum efficiency was estimated by placing the phosphor between the $x$-ray tube and an ion chamber, and measuring the relative $\mathrm{x}$-ray transmission through the phosphor.

\begin{tabular}{|c|c|c|c|c|}
\hline Phosphor & $\begin{array}{l}\text { Commercial } \\
\text { Name }\end{array}$ & $\begin{array}{l}\text { Spectral } \\
\text { Range (nm) [30] }\end{array}$ & $\begin{array}{l}\text { Relative } \\
\text { Light Output }\end{array}$ & $\begin{array}{l}\text { Quantum Eff. } \\
\text { at } 28 \mathrm{kVp} \dagger\end{array}$ \\
\hline $\mathrm{BaFCl}: \mathrm{Eu}$ & Dupont Quanta II & blue $350-450$ & $8 \overline{2 \%}$ & $80 \%$ \\
\hline CsI:Tl & $\mathrm{N} / \mathrm{A}$ & white $400-700$ & $68 \%$ & $99 \%$ \\
\hline $\mathrm{Gd}_{2} \mathrm{O}_{2} \mathrm{~S}: \mathrm{Pr}$ & $\mathrm{N} / \mathrm{A}$ & green 510 & $80 \%$ & $97 \%$ \\
\hline $\mathrm{Gd}_{2} \mathrm{O}_{2} \mathrm{~S}: \mathrm{Tb}$ & Kodak Min-R Medium & green 500 & $100 \%$ & $78 \%$ \\
\hline LaOBr:Tm & MCI-Optonix & biue $300-480$ & $92 \%$ & $84 \%$ \\
\hline $\mathrm{YTaO}_{4}$ & MCI-Optonix & $\mathrm{u} / \mathrm{v}$-blue $280-420$ & $18 \%$ & $92 \%$ \\
\hline $\mathrm{ZnCdS:Ag}$ & MCI-Optonix & green $450-650$ & $290 \%$ & $79 \%$ \\
\hline $\mathrm{B} 90$ & Kodak (proprietary) & blue & $44 \%$ & $82 \%$ \\
\hline
\end{tabular}

Table 2.1: List of the phosphors tested and some of their properties. $f$ - Efficiencies approximate only (no correction for absorption of backing layer)

The short pulse response curves are shown in Figure 2.7. These curves were fit to Equation 2.7 using the iterative convolution technique described above. The impulse response, $I(t)$, obtained from each of the fits is shown in Figure 2.8. The number of components, $N$, was determined by repeating the fits for $N=1,2,3, \ldots$ and selecting the value of $N$ that produced the $\chi_{R}^{2}$ value that fell in or nearest to the acceptable range of 0.8 to 1.2 . The resultant values for the luminescence lifetimes are shown in Table 2.2. The $90 \%$ confidence values shown in brackets next to each result. As a comparison, Table 2.3 has a partial list of lifetimes measured by others. Each row in that table indicates a separate measurement. 


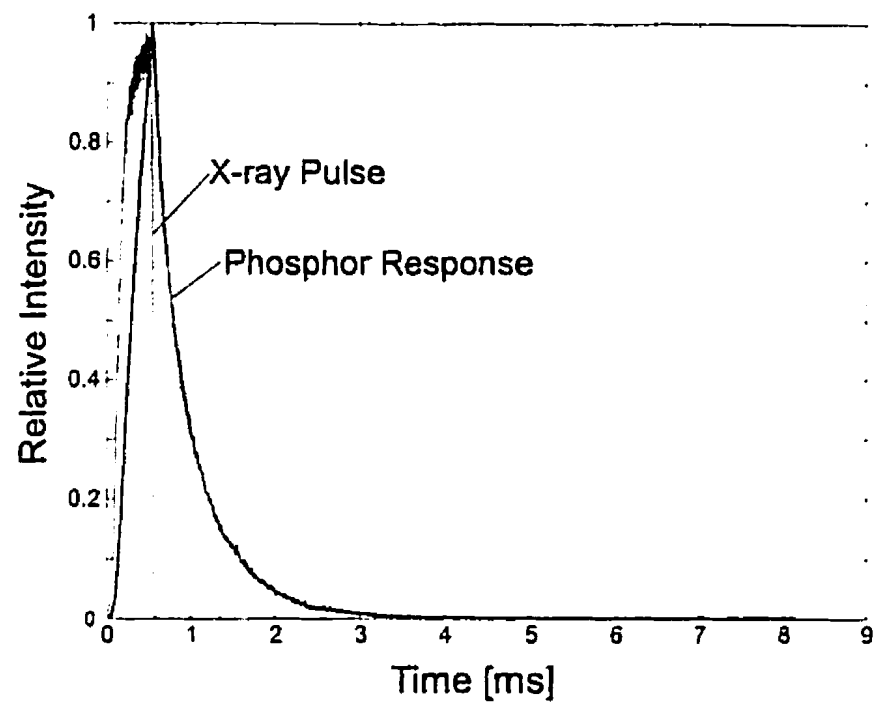

Figure 2.6: An example of the data collected from the short pulse response experiment. The phosphor used in this example is $\mathrm{Gd}_{2} \mathrm{O}_{2} \mathrm{~S}: \mathrm{Tb}$.

All of the phosphors were fitted with two or more components. Except for $\mathrm{Gd}_{2} \mathrm{O}_{2} \mathrm{~S}$ : $\mathrm{Tb}$, all the phosphors exhibited a dominant lifetime component that was less than $20 \mu \mathrm{s}$. The multiple number of components seems to agree with qualitative observations made by others[42] regarding the complexity of the luminescence response of many $x$-ray phosphors. The $90 \%-10 \%$ fall-time of the $\mathrm{x}$-ray pulse was $26 \mu \mathrm{s}$ which may have caused systematic overestimates of very short lifetimes components $(<10 \mu \mathrm{s})$. For very short lifetimes, the phosphor persistence OTF will be approximately flat for all but the most extreme velocities. Any overestimate associated with these short lifetime components is expected to cause minimal change in the OTF. The phosphors also showed an upper limit of about $4400 \mu \mathrm{s}$. It is possible that the longest components are underestimated because the data lengths were limited to $7000 \mu$ s following $x$-ray shutoff. As well, these very long components may actually be a result of afterglow (i.e. luminescence following trapping) rather than 'true' exponential luminescence. Except for $\mathrm{ZnCdS}: \mathrm{Ag}$ and $\mathrm{YTaO}_{4}: \mathrm{Tm}$, the longest components contributed to less than $2 \%$ of the total and thus these components are expected to have a negligible 

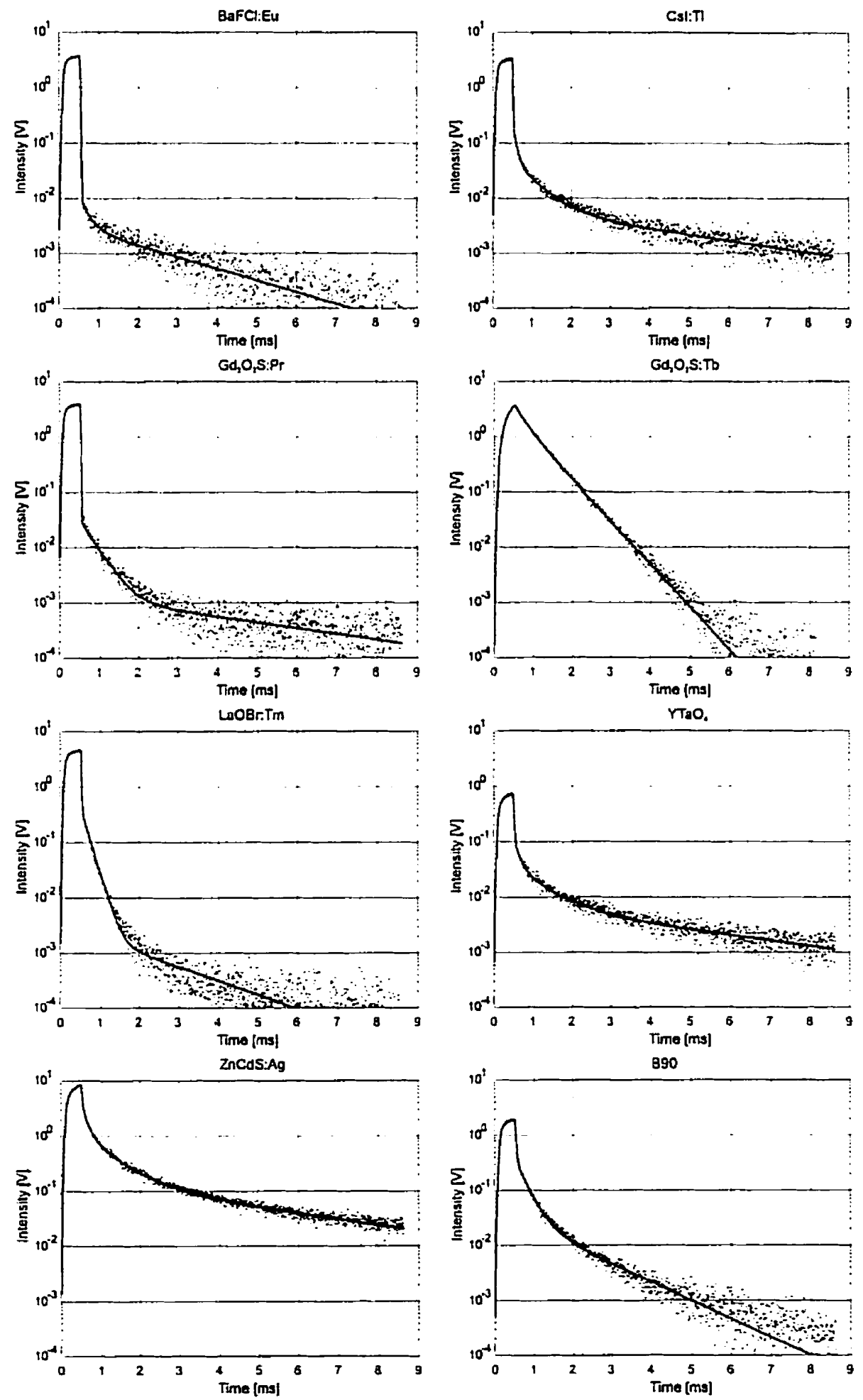

Figure 2.7: Fitted short pulse response luminescence curves for eight phosphors. Solid line is the fitted curve corresponding to the parameters in Table 2.2. Only every 10th data point is shown for clarity. 

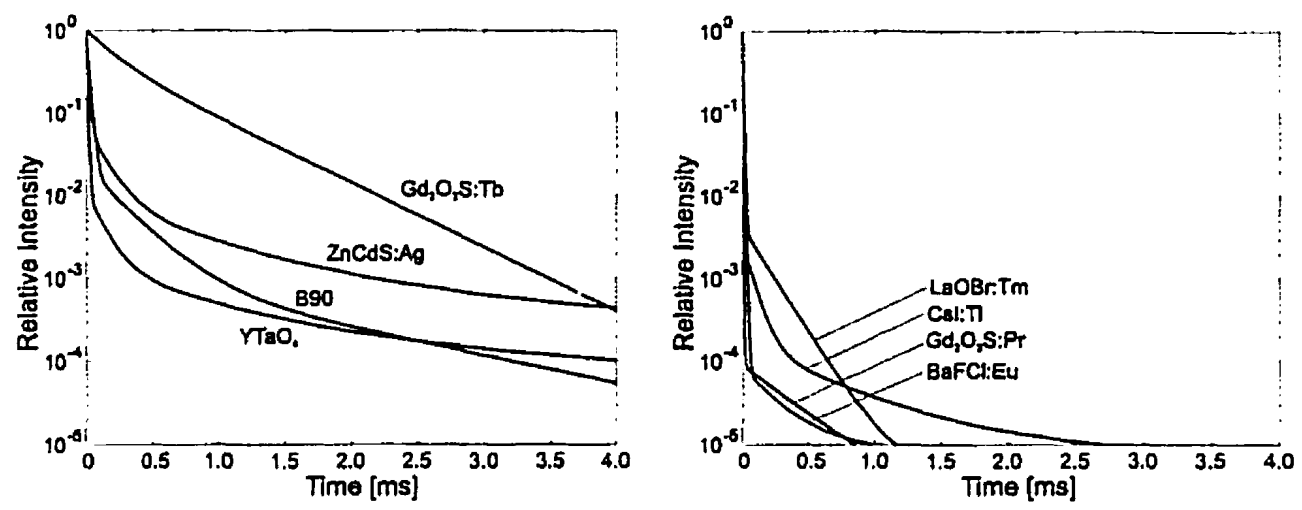

Figure 2.8: Luminescence impulse response for parameters in Table 2.2. The phosphors have been divided into the four slowest responses and four fastest responses

contribution to the persistence OTF. As will be seen in the next section, the $\mathrm{ZnCdS:Ag}$ and $\mathrm{YTaO}_{4}: \mathrm{Tm}$ have a large afterglow signal, and the longest components have a noticeable contribution to the persistence OTF.

To demonstrate the effect of the luminescence response, the magnitude component predicted by Equation 2.2 is shown in Figure 2.9 for scanning velocities of 40 and $80 \mathrm{~mm} / \mathrm{s}$ for all the phosphors tested. Figure 2.10 shows the corresponding PTF curves.

\subsubsection{Afterglow Measurements}

The long pulse measurements are shown in Figure 2.11. All phosphors demonstrated an increase in the long components following the $500 \mathrm{~ms}$ pulse. These long components were assumed to be afterglow following the algebraic behaviour of Equation 1.17. The measurement of long components was limited by the intrinsic response of the photomultiplier. The PMT demonstrated its own persistence. This is probably due to trapping sites within the glass of the photocathode[36, 33]. This limit is shown in Figure 2.11 by the dashed line. The limit was determined by measuring the response of the PMT to a very sharp $500 \mathrm{~ms}$ pulse of light from a green LED source. This only applies to the long pulse data, during short pulse excitation, the intrinsic persistence of the PMT was negligible. 


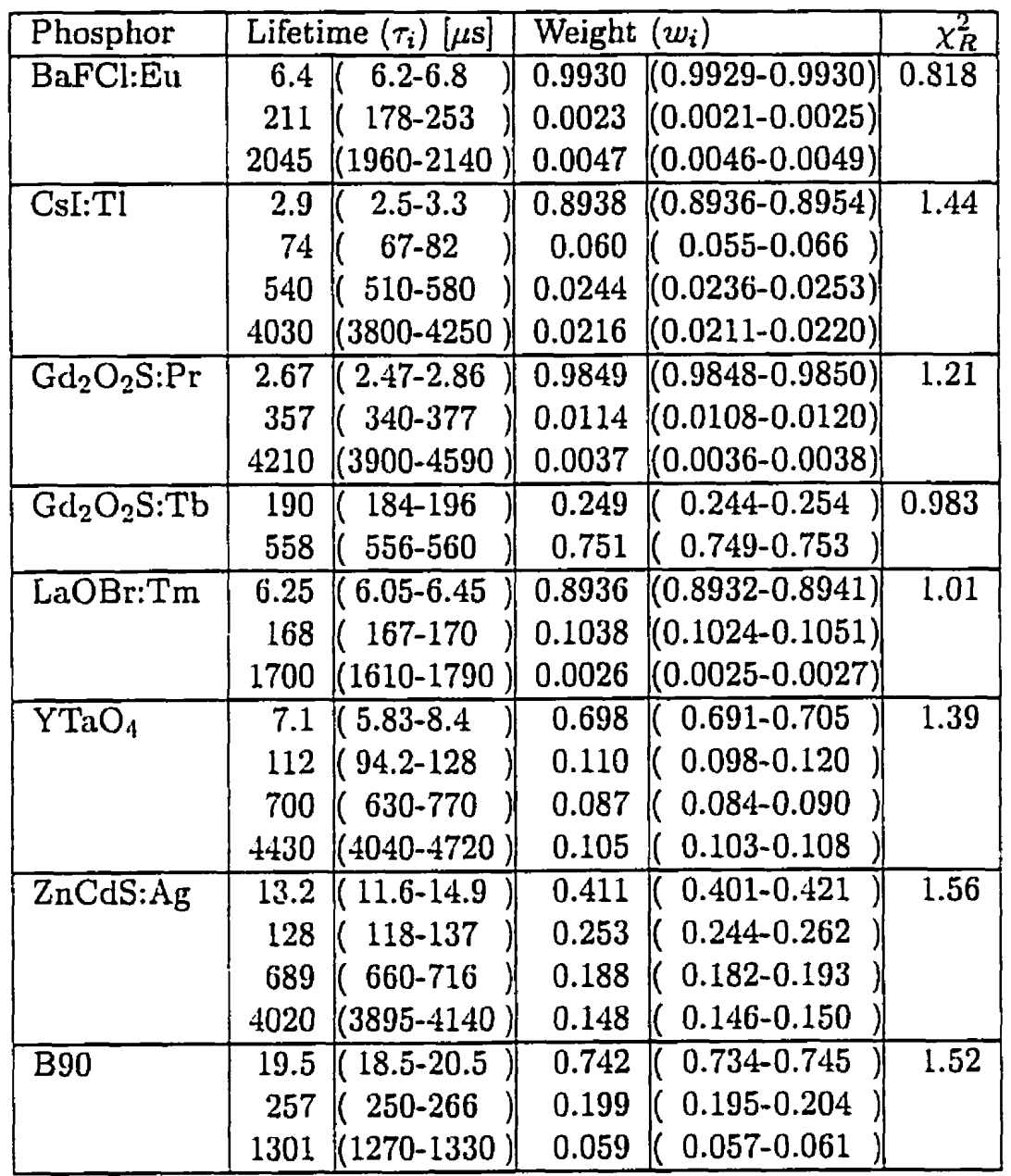

Table 2.2: Fitted parameters for the phosphors tested. The bracketed numbers are the approximate $90 \%$ confidence intervals for each parameter

\begin{tabular}{|l|l|l|}
\hline Phosphor & Lifetime $[\mu \mathrm{s}]$ & Reference \\
\hline $\mathrm{CsI}: \mathrm{Tl}$ & 1.1 & {$[43]$} \\
& $0.8 \pm 0.1$ & {$[44]$} \\
& 0.98 & {$[30]$} \\
& $0.60 \pm 0.05(54 \%) / 3.4 \pm 0.5(46 \%)$ & {$[45]$} \\
\hline $\mathrm{Gd}_{2} \mathrm{O}_{2} \mathrm{~S}: \mathrm{Pr}$ & 3 & {$[30]$} \\
\hline $\mathrm{Gd}_{2} \mathrm{O}_{2} \mathrm{~S}: \mathrm{Tb}$ & 400 & {$[46]$} \\
& 700 & {$[33]$} \\
\hline
\end{tabular}

Table 2.3: Lifetime measurements from literature 

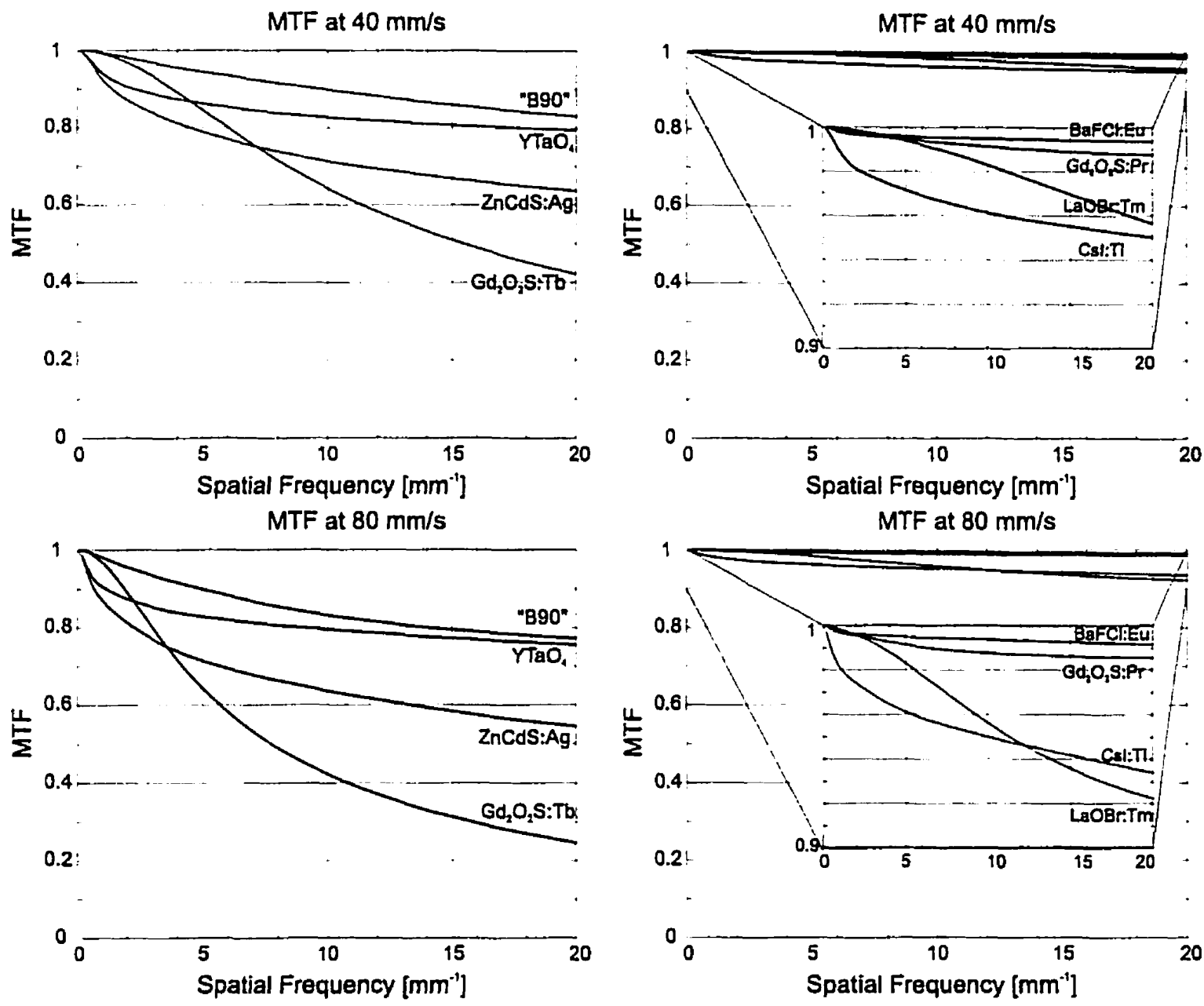

Figure 2.9: MTF component due to phosphor persistence. The curves are shown at $40 \mathrm{~mm} / \mathrm{s}$ (top) and $80 \mathrm{~mm} / \mathrm{s}$ (bottom)

An earlier work by Shepherd et. al[33] measured the short pulse and step response of two of the phosphors: $\mathrm{Gd}_{2} \mathrm{O}_{2} \mathrm{~S}: \mathrm{Tb}$ and $\mathrm{Gd}_{2} \mathrm{O}_{2} \mathrm{~S}: \mathrm{Pr}$. Estimates of selected points from Figures 2-4 of Shepherd's work are compared to the responses measured in the present work. The comparison is shown in Figure 2.12. Qualitatively, the two sets of experiments appear to agree well for $\mathrm{Gd}_{2} \mathrm{O}_{2} \mathrm{~S}$ : Tb and only slightly less well for $\mathrm{Gd}_{2} \mathrm{O}_{2} \mathrm{~S}: \mathrm{Pr}$. Also shown is a comparison of the CsI:Tl response to data presented by Grabmaier [30], who measured a much higher afterglow level. The differences in response between the results of this work and the responses measured by Shepherd and Blasse are 

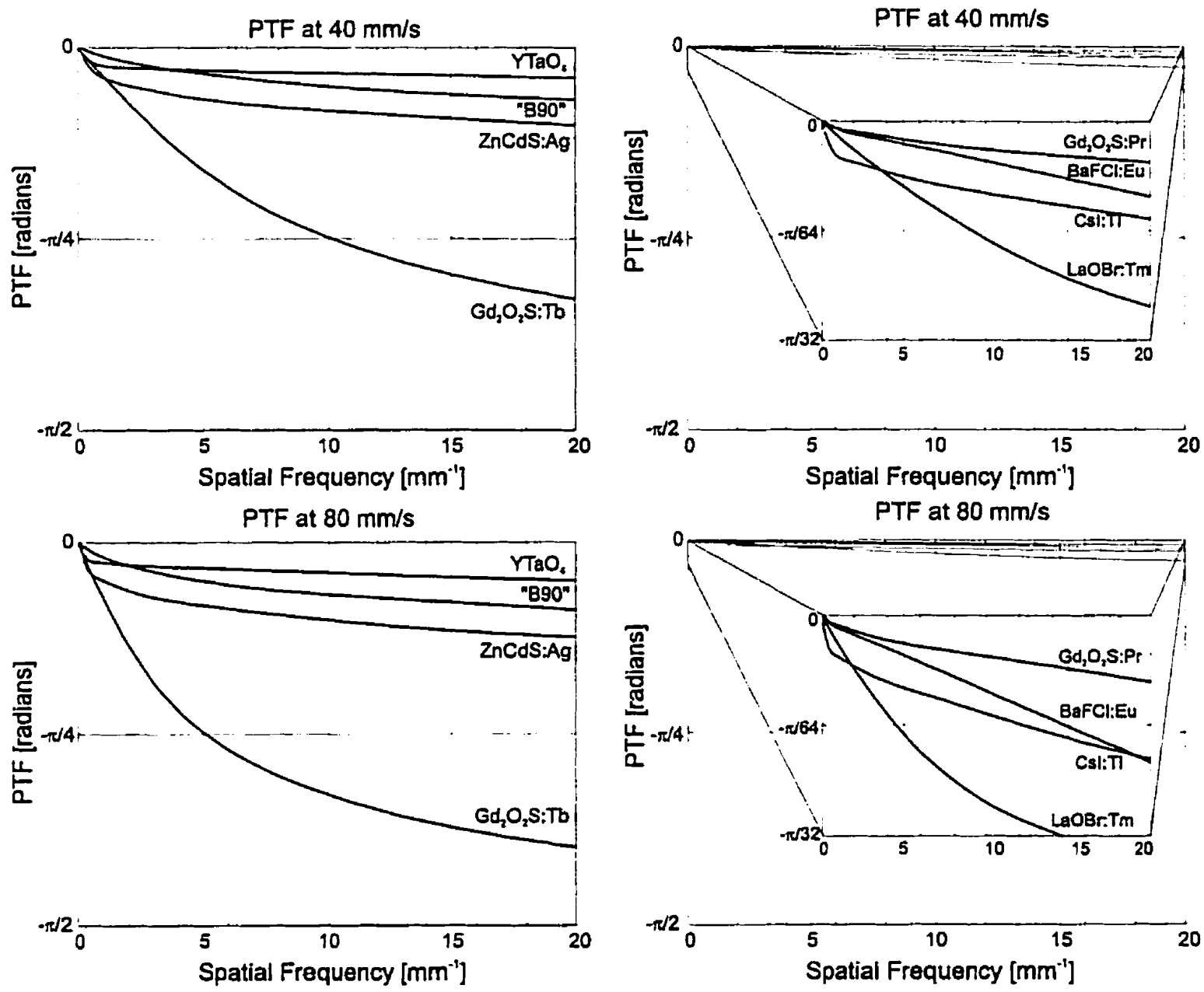

Figure 2.10: PTF component due to phosphor persistence. The curves are shown at $40 \mathrm{~mm} / \mathrm{s}$ (top) and $80 \mathrm{~mm} / \mathrm{s}$ (bottom)

partly due to different $\mathrm{x}$-ray pulse widths and different sensitivities in the photodetectors used for each experiment. Grabmaier has also noted that phosphors of identical chemical composition, but differing manufacturing techniques can have different levels of afterglow[34].

Equation 2.11 was fitted to the long pulse response of $\mathrm{ZnCdS:Ag,} \mathrm{YTaO}_{4}, \mathrm{CsI} \mathrm{Tl}$, and $\mathrm{Gd}_{2} \mathrm{O}_{2} \mathrm{~S}: \mathrm{Pr}$. The parameters obtained are shown in Table 2.5. All of the fits were done over a range that began at least $1 \mathrm{~ms}$ after the end of the $\mathrm{x}$-ray pulse.

The non-linear least squares technique proved to be very slow reaching a minimum requiring 

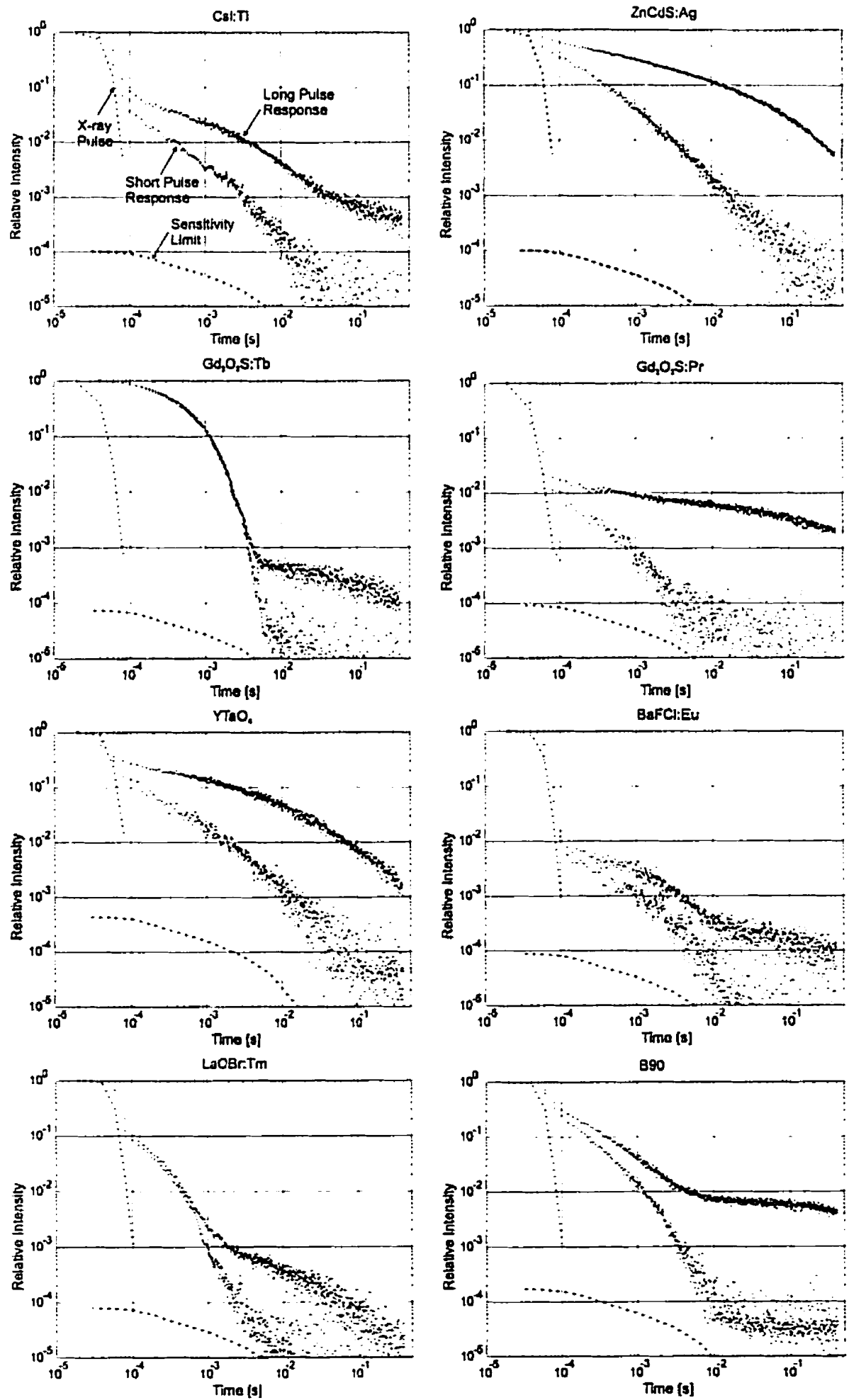

Figure 2.11: Short $(500 \mu \mathrm{s})$ and long pulse $(500 \mathrm{~ms})$ response measurements. The number of data points shown has been reduced clarity. The dashed line corresponds to the sensitivity limit of the PMT due to its own persistence, and the dotted line corresponds to the falling edge of the $x$-ray pulse. 

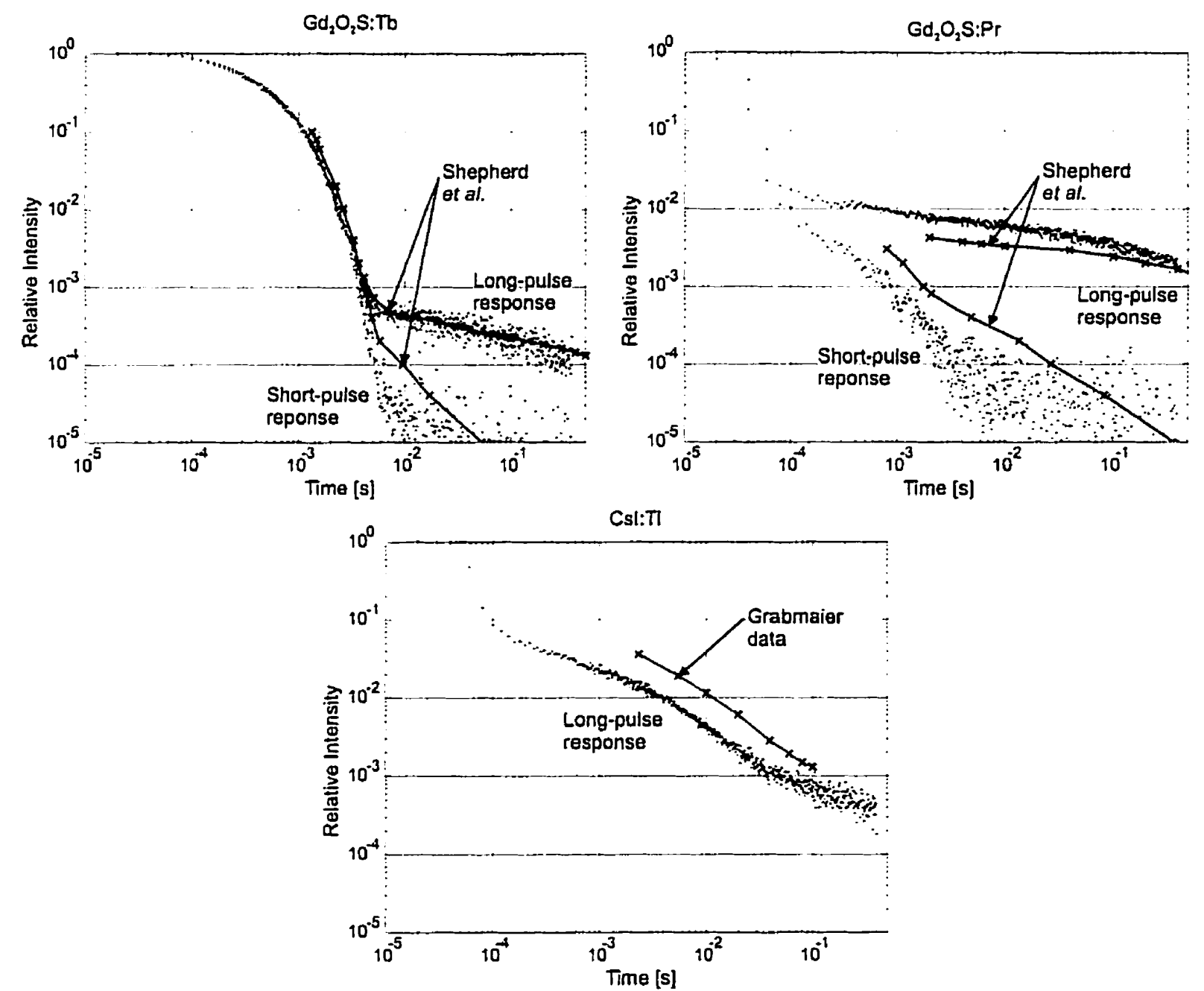

Figure 2.12: Comparison of $\mathrm{Gd}_{2} \mathrm{O}_{2} \mathrm{~S}$ : $\mathrm{Tb}$ and $\mathrm{Gd}_{2} \mathrm{O}_{2} \mathrm{~S}: \mathrm{Pr}$ long and short pulse response to selected data points from Shepherd et. al. Also shown is comparison of long pulse response for CsI:Tl to data from Grabmaier. Points are straight line connected for clarity. 

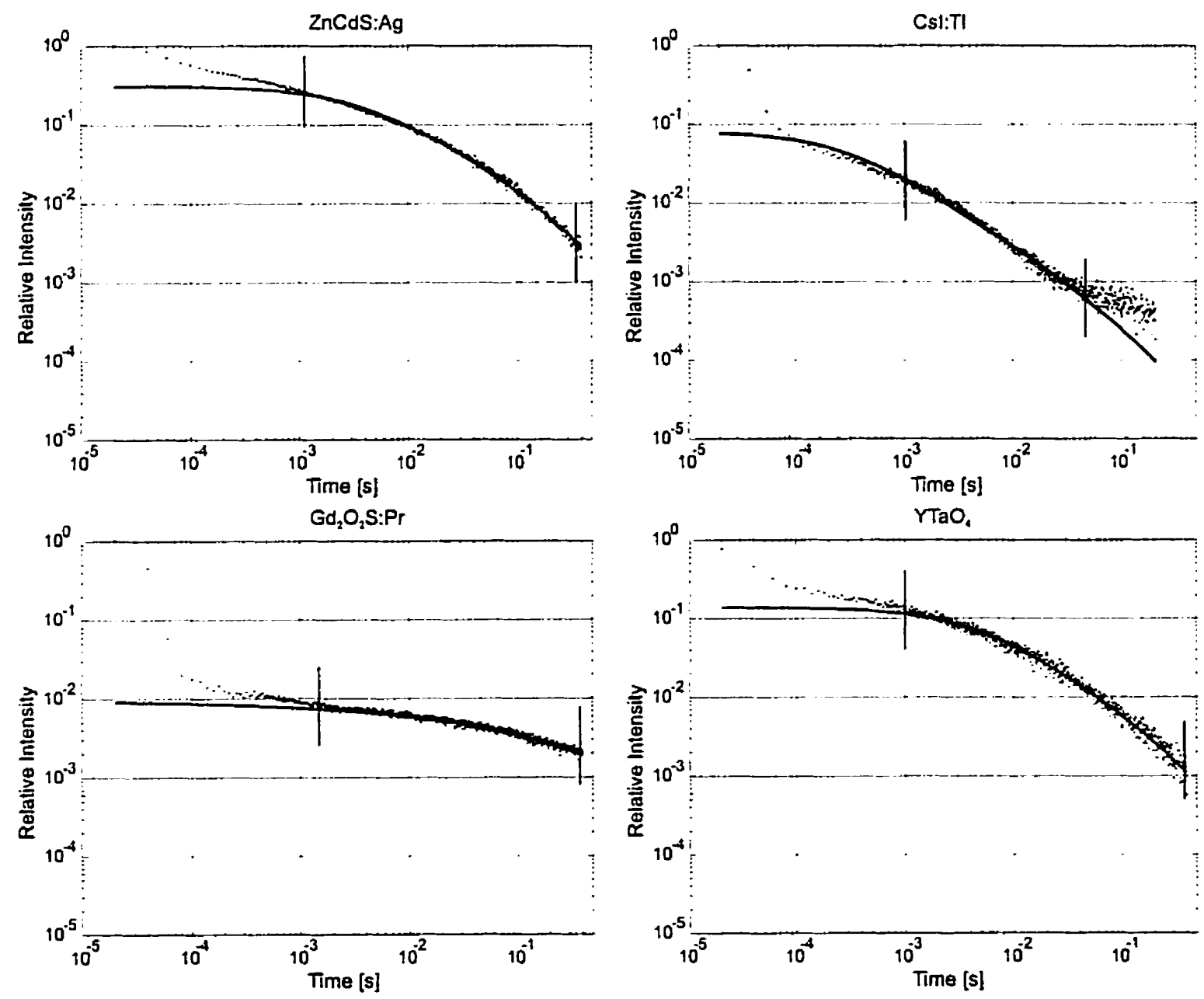

Figure 2.13: Afterglow regression curves for $\mathrm{ZnCdS}: \mathrm{Ag}, \mathrm{CsI}: \mathrm{Tl}, \mathrm{Gd}_{2} \mathrm{O}_{2} \mathrm{~S}: \mathrm{Pr}$ and $\mathrm{YTaO}_{4}$. The range over which the fit was performed lies between the two vertical bars. 


\begin{tabular}{|r|l|l|l|l|}
\hline & \multicolumn{4}{|c|}{ Time to Relative Intensity [ms] } \\
Phosphor & $10^{-1}$ & $10^{-2}$ & $10^{-3}$ & $10^{-1}$ \\
\hline $\mathrm{BaFCl}$ Eu (long) & $0.079 \dagger$ & 0.11 & 3.6 & $\approx 960$ \\
(short) & 0.070 & 0.10 & 0.76 & 5.7 \\
\hline $\mathrm{CsI}: \mathrm{TI}$ & 0.089 & 4.3 & 46 & $>8000 ?$ \\
& 0.074 & 0.39 & 3.6 & 18 \\
\hline $\mathrm{Gd}_{2} \mathrm{O}_{2} \mathrm{~S}: \mathrm{Tb}$ & 1.1 & 2.4 & 4.3 & 130 \\
& 1.1 & 2.4 & 3.6 & 4.5 \\
\hline $\mathrm{Gd}_{2} \mathrm{O}_{2} \mathrm{~S}: \mathrm{Pr}$ & 0.053 & 0.49 & $\approx 1000$ & $>4 \cdot 10^{4} ?$ \\
& 0.044 & 0.088 & 0.9 & 6.5 \\
\hline $\mathrm{LaOBr}^{\mathrm{T}} \mathrm{Tm}$ & 0.089 & 0.44 & 2.0 & 62 \\
& 0.079 & 0.44 & 1.0 & 3 \\
\hline $\mathrm{YTaO}$ & 2.0 & 70 & $\approx 1000$ & $>10^{4} ?$ \\
& 0.13 & 2.0 & 16 & 113 \\
\hline $\mathrm{ZnCdS:Ag}$ & 14 & 275 & $\approx 1600$ & $>6000 ?$ \\
& 0.39 & 3.2 & 18 & 125 \\
\hline $\mathrm{B} 90$ & 0.39 & 4.3 & $\approx 3500$ & $>5000 ?$ \\
& 0.16 & 1.2 & 2.6 & 10.4 \\
\hline & & & & \\
\hline
\end{tabular}

Table 2.4: Afterglow times following the step response (long) and the short pulse response. Times greater than $500 \mathrm{~ms}$ are based on extrapolation of the curves in Figure 2.11. $\dagger$ - Very short times $<0.1 \mathrm{~ms}$ for all phosphors are dominated by the fall-time of the $x$-ray pulse.

more than 1000 iterations for the afterglow model. Despite reasonably good $\chi_{R}^{2}$ values (see Table 2.5 ) for the four phosphors, the curve fits did not appear to be be entirely satisfactory. The fits were sensitive to the range over which the fit was performed. This suggests that Equation 2.11 was a poor or insufficient model[29]. However, despite the difficulties with the fits, they are instructive in determining the approximate effect afterglow can have on image quality in the scanning system.

In the short pulse measurements, both $\mathrm{YTaO}_{4}$ and $\mathrm{ZnCdS}: \mathrm{Ag}$ appeared to have some long components (4430 at $10 \%$ and 4020 at $15 \%$ ). However, following the long pulse measurements, afterglow appears to be very large in both of these phosphors, and it was believed that these components were actually part of the afterglow response. To demonstrate the significance of afterglow; the MTF was calculated based on Equation 2.14, using the inverse power law parameters and the primary exponential parameters with lifetimes $<600 \mu \mathrm{s}$. The MTFs obtained are shown in Figure 2.14 for a scanning velocity of $v=40 \mathrm{~mm} / \mathrm{s}$, compared to MTFs calculated from the weighted 


\begin{tabular}{|c|c|c|c|c|}
\hline Phosphor & \multicolumn{2}{|c|}{ Parameters } & $\begin{array}{r}\text { Relative } \\
\text { Intensity } \\
(1-\beta)\end{array}$ & $\begin{array}{r}\text { Reduced } \\
\text { Chi-Square } \\
\chi_{R}^{2}\end{array}$ \\
\hline CsI:Tl & 3074 & 1.853 & 0.008 & 1.39 \\
\hline $\mathrm{Gd}_{2} \mathrm{O}_{2} \mathrm{~S}: \mathrm{Pr}$ & $1.096 \times 10^{8}$ & 0.793 & 0.010 & 1.14 \\
\hline $\mathrm{YTaO}_{4}$ & 245.0 & 1.919 & 0.138 & 1.32 \\
\hline ZnCdS:Ag & 300.0 & 1.832 & 0.314 & 0.93 \\
\hline
\end{tabular}

Table 2.5: Regression parameters obtained from the inverse power law model for afterglow
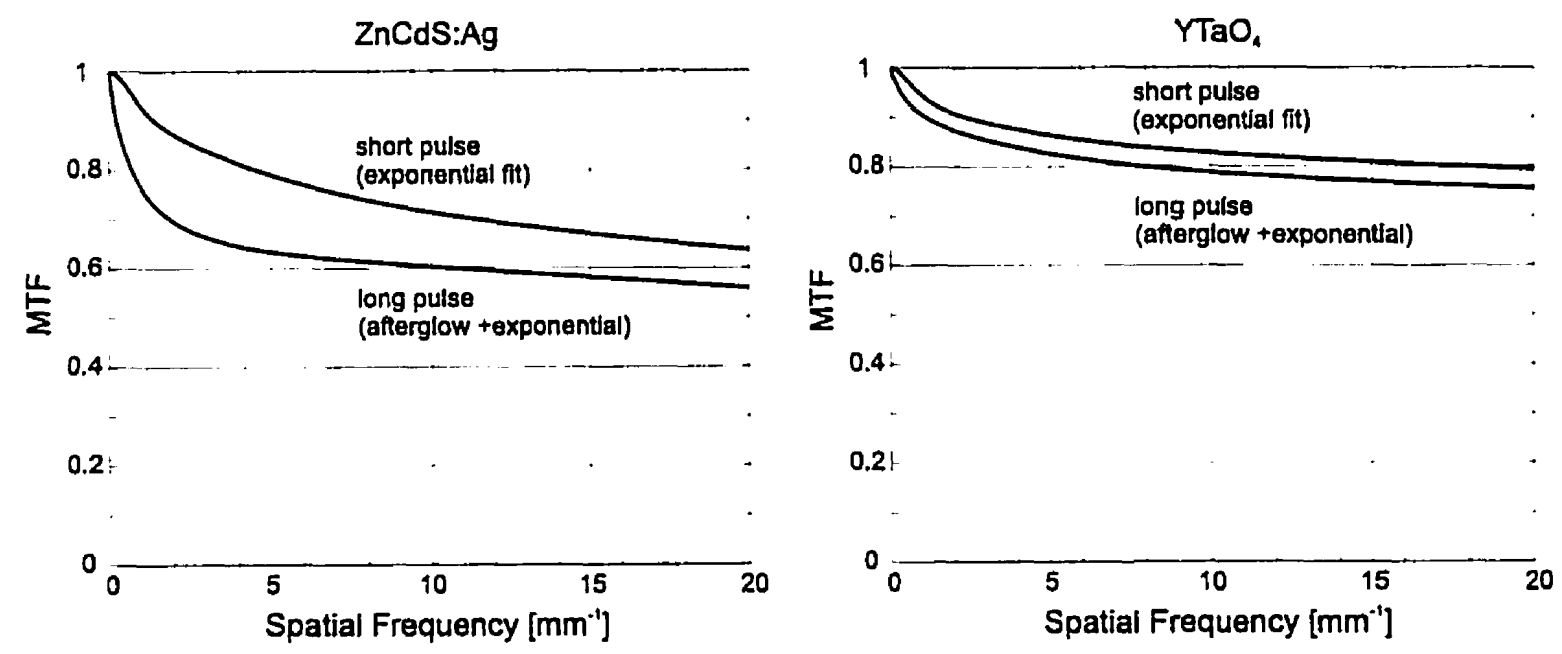

Figure 2.14: Comparison of MTF curves calculated from exponential fit of the short pulse response and the MTF curves calculated from the combined response of the short exponential lifetimes and the inverse power law afterglow measured in the long pulse response.

exponentials (Equation 2.2) determined from the short pulse response. Note that the PTF is not shown for the two cases, but a similar change in the phase term is also expected.

Of the eight phosphors tested, $\mathrm{ZnCdS}: \mathrm{Ag}$ and $\mathrm{YTaO}_{4}: \mathrm{Tm}$ showed the most dramatic increase in afterglow intensity. The afterglow of these two phosphors was large enough to cause significant degradation in the MTF compared to the MTF determined from the short pulse exponential fits. The rest of the phosphors showed much smaller differences between the MTF predicted by the set of exponentials and the MTF predicted by including the inverse power law fit.

The effect of the afterglow for $\mathrm{ZnCdS}: \mathrm{Ag}$ appears as a sharp drop in MTF at low spatial frequency. This low-frequency drop is very similar to the effect of veiling glare[47, 48] observed in 
image intensifier tubes. Veiling glare occurs as a result of light scattering in the phosphor screen and scattering back from the metal components inside the intensifier tube. Veiling glare results in a low frequency drop which, in turn, causes a significant reduction in contrast. Afterglow can be considered as a light scattering phenomenon, and thus it is expected to cause a similar contrast degradation.

\subsection{Discussion}

\subsubsection{Relative Significance}

The amount of image degradation induced by phosphor persistence can be reduced by lowering the scan speed. However, lower scan speeds greatly increase the likelihood of image degradation due to patient motion.

As demonstrated in Figure 2.9, phosphor persistence can introduce some resolution loss in the scanning direction. The impact of persistence in a TDI system can be compared to the various other blurring mechanisms that occur. Consider a TDI scanning system consisting of a CCD with 64 columns in the TDI direction, a four phase clock and a scanning rate of $40 \mathrm{~mm} / \mathrm{s}$. The CCD is coupled by a fibre optic taper to a Min R-Medium $\left(\mathrm{Gd}_{2} \mathrm{O}_{2} \mathrm{~S}\right.$ :Tb) screen and the pixel pitch at the phosphor plane is $50 \mu \mathrm{m}$. A number of image blurring mechanisms for this system are listed in Table 2.6.

The fundamental limit of resolution for a digital system is set by the pixel aperture. Light scattering in the phosphor further reduces the resolution and is probably the biggest limiting factor for resolution. The coupling optics also introduce significant image degradation.

Motion effects, as mentioned in Chapter I, also cause blurring in the scan direction for the TDI system. However, with careful matching of the charge transfer rate to the velocity (to within $\pm 1 \%$ with stepper motors and programmable clock rates) and carefully designed CCDs, the degradation is quite small. Charge transfer inefficiencies are quite low for modern CCDs 


\begin{tabular}{|l|l|l|}
\hline Blurring Effect & MTF at 5 $\mathrm{mm}^{-1}$ & $10 \mathrm{~mm}^{-1}$ \\
\hline \multicolumn{2}{|c|}{ Stationary Effects } \\
\hline Pixel Aperture Response & 0.9 & 0.63 \\
Phosphor Resolution[21] & 0.4 & 0.15 \\
Coupling Optics[21] & 0.7 & 0.40 \\
\hline \multicolumn{2}{|c|}{ Motion Related Effects } \\
\hline Clock Structure & 0.98 & 0.93 \\
Velocity Mismatch of 1\% & 0.996 & 0.93 \\
Charge Transfer Inefficiency $\epsilon=10^{-4}$ & 0.975 & 0.950 \\
Phosphor Persistence & 0.84 & 0.62 \\
\hline
\end{tabular}

Table 2.6: Comparison of various blurring mechanisms and their relative impact at 5 and $10 \mathrm{~mm}^{-1}$ $\left(<10^{-4}[23]\right)$. From the values shown in Table 2.6, phosphor persistence is the most important motion-related mechanism of resolution loss and, at least, for $\mathrm{Gd}_{2} \mathrm{O}_{2} \mathrm{~S}$ : $\mathrm{Tb}$ the relative amount of loss in the MTF is as large as the losses resulting from the aperture response and the coupling optics. Note that this analysis considered the MTF only.

\subsubsection{Afterglow Revisited}

It must be noted that in the simple step response experiment, it was arbitrarily assumed that afterglow (inverse power law) occured, as opposed to a very long primary exponential component. Certainly, the dramatic increase in intensity of the longest components for $\mathrm{ZnCdS}: \mathrm{Ag}_{1}, \mathrm{YTaO}_{4}: \mathrm{Tm}$, $\mathrm{CsI}: \mathrm{Tl}, \mathrm{Gd}_{2} \mathrm{O}_{2} \mathrm{~S}: \mathrm{Pr}$, and $\mathrm{B} 90$ suggest that a non-linear effect has occurred, indicating afterglow. Because of this non-linearity, the MTF curves calculated for Figure 2.14 represent the linear systems approximation for the $x$-ray intensity used in the experiment. If very high intensities were expected, then afterglow could potentially become even more damaging to image quality.

The long components of $\mathrm{ZnCdS}: \mathrm{Ag}_{\mathrm{g}}$ and $\mathrm{YTaO}_{4}: \mathrm{Tm}$ were remodeled as a sum of exponentials, requiring at least three $(N=3)$ components to provide a satisfactory fit of the long tail. This would mean a total of six or more components to completely describe the response, including the shortest and longest components. Although this is not impossible, the assumption of six independent transitions seems to be very unlikely. Because of the correlation between the terms in a weighted 
exponential fit, the accuracy of the terms (six lifetimes and six weights) becomes very poor. If the signal that was assumed to be afterglow was actually composed of several long primary exponential components that were too weak to measure with the short pulse measurement, these components should show a linear response with intensity. If this is the case, then the inverse power law fit represents an approximation to the luminescence response. Thus, even if the assumption of the presence of afterglow is invalid, the MTF curves calculated for Figure 2.14 must be, at least, an empirical approximation of the blurring expected for the system. In fact, if the "afterglow" is a primary luminescence with linear behaviour, then the empirical MTF will be valid over all imaging conditions as opposed to the original assumption that the MTF is only a representative curve for the blur expected due to afterglow under imaging conditions similar to the conditions of the luminescence experiment.

If it is desired to completely analyze the afterglow, a much more rigorous analysis would be required. Some of the experiments that could be performed to determine the afterglow response, would include a thermoluminescence or a spectrographic analysis to determine the distribution of the energy levels of the traps in the crystal[32]. As well, afterglow can be identified more precisely by taking advantage of some of its properties. Since afterglow can vary non-linearly as a function of excitation intensity[33], and since afterglow is altered by the presence of other impurities and even manufacturing techniques[30,31,34], these properties could be analyzed to help isolate and characterize the afterglow response.

Regardless of the actual behaviour of afterglow, the long term luminescence can influence low spatial frequency contrast. Thus, there must be a maximum acceptable level of afterglow intensity for a scanning system. Grabmaier suggests that for CT, the afterglow should be less than $0.01 \%$ within $3 \mathrm{~ms}[30]$. This is a very strict criterion because of the very fast scanning speeds used for $\mathrm{CT}$, and need for accurate signals to perform the CT reconstructions. For a radiography system that uses a scanning detector, the criterion is less restrictive.

It seems reasonable to suggest that the phosphor should have low afterglow levels to prevent 
contrast loss for low intensity objects (i.e. most anatomical tissues). For example consider a scanning system designed specifically for mammography. The dynamic range of intensity required for mammography is between a factor of 10 and 100[3] between the background (air) and the breast. At the low end of this range, low contrast structures must be visible. Contrast between two objects (or an object and the background) is defined as:

$$
C=\frac{A-B}{A+B}
$$

where $A$ is the signal for object 1 , and $B$ is the signal for object 2. Yaffe[3] suggests that for mammography, the ability to see a $1 \%$ decrease in signal is desirable for lesion detection. Thus at a factor of 100 below maximum intensity; object 1 has a signal of $A=0.100$ and $B=0.099$. This corresponds to a contrast of $C=0.005$. In the presence of afterglow, however, the contrast will be reduced. Assume that the afterglow level is $\delta$ and this adds signal to both $A$ and $B$. The new contrast will be

$$
\begin{aligned}
C^{\prime} & =\frac{(A+\delta)-(B+\delta)}{(A+B+2 \delta)} \\
& =\frac{A-B}{(A+B+2 \delta)} .
\end{aligned}
$$

In order to maintain good contrast, assume that this new contrast can be no lower than $C^{\prime}=f \cdot C$ where $f$ is a constant $<1$. For example, if a twenty percent reduction in contrast at low signal is acceptable, $f=0.8$. Using the values of $A$ and $B$ above and solving for $\delta$, gives maximum afterglow level of $0.25 \%$.

The time than can be tolerated for the afterglow to fall to this level will depend on the object being imaged. For example, consider the system designed for mamography. Figure 2.15 shows the simple geometry of a breast that is uniformly compressed for a mammographic exam. The signal is lowest behind the uniform region. From the figure, the transition from maximum signal to minimum signal is approximately equal to one half the compression thickness, $d$. If the 


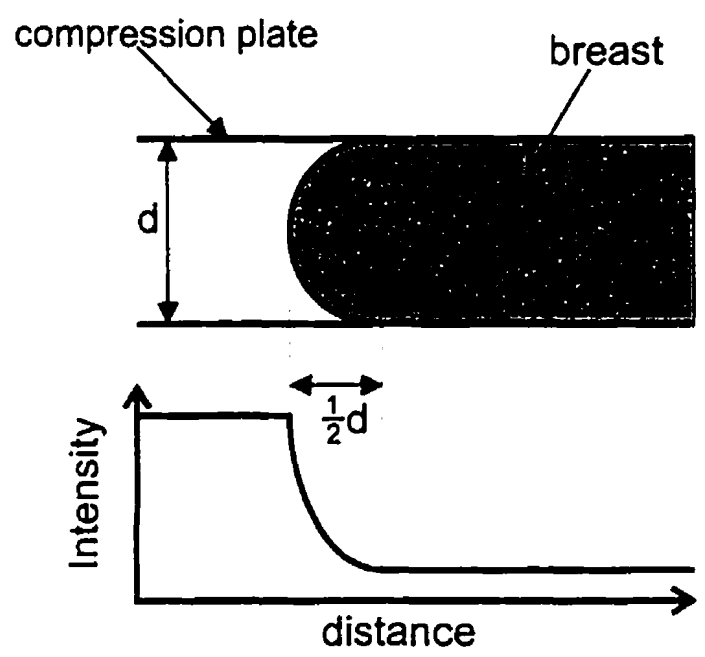

Figure 2.15: Simple geometric model of a compressed breast. The $\mathrm{x}$-ray signal reaching the detector is plotted as a function of distance. The transition from maximum to minimum signal is approximately 0.5 the compression thickness.

average compressed breast is $4 \mathrm{~cm}$ thick, then the scan distance from maximum to minimum will be $2 \mathrm{~cm}$. The time allowed for afterglow to fall to $\delta$ is simply $\frac{d}{2 \cdot v}$ for a scanning system with a velocity, $v$. For a scan rate of $60 \mathrm{~mm} / \mathrm{s}$, this would give a time of $400 \mathrm{~ms}$ for the afterglow to fall to $0.25 \%$

Of the phosphors tested, $\mathrm{YTaO}_{4}$ and $\mathrm{ZnCdS}: \mathrm{Ag}$, and $\mathrm{B} 90$ fail this limit, and $\mathrm{Gd}_{2} \mathrm{O}_{2} \mathrm{~S}: \mathrm{Pr}$ is just borderline. Of course, the afterglow level, velocity and geometry have been arbitrarily set. Depending on the application, required scan rate, and acceptable contrast levels, the maximum acceptable afterglow requirements will vary.

\subsection{Conclusion}

This chapter developed a model describing the blurring mechanism in a TDI scanning system, induced by luminescence response of $x$-ray phosphors. Because of the asymmetric nature of this blur, a complete OTF must be considered in determining image quality, rather than amplitude (MTF) alone. The primary luminescence was modeled with a sum of weighted exponentials and 
an iterative deconvolution technique was used to compensate for the $\mathrm{x}$-ray pulse shape. Results showed that if the lifetimes of the phosphor are greater than a few hundred microseconds then there can be significant blurring introduced in the scanning direction. As well, the long term afterglow was measured following a much longer pulse of radiation. Very high levels of afterglow cause a low frequency drop in the MTF similar to veiling glare. Only ZnCdS:Ag demonstrated afterglow levels high enough to cause a significant degradation in the MTF compared to that predicted by considering the primary luminescence alone. Because afterglow can potentially reduce contrast in anatomic regions where the signal has dropped by 1-2 orders of magnitude below the background signal, only phosphors that demonstrate an afterglow that drops rapidly to a low intensity should be used in a scanning system. Careful selection of the best phosphor for x-ray scanned imaging requires consideration of not only the primary luminescence response but the long term afterglow as well. 


\section{Chapter 3}

\section{Future Work and Summary}

In the previous chapter, a model was developed to describe the loss of spatial resolution caused by phosphor persistence in an $x$-ray scanning system. A measurement technique was refined for measuring the decay parameters for both primary decay and afterglow. Knowing the influence of persistence on image quality provide an additional tool in determining an suitable phosphor for use in a scanning system.

Persistence produces an asymmetric effect, and has interesting consequences on image quality determination, requiring use of the OTF rather than the traditional use of the MTF for correct characterization. In Chapter II, this was demonstrated when the persistence OTF was considered alone. The impact of this asymmetry on a realistic imaging system also needs to be studied.

Ultimately, phosphor based systems may be replaced by other detector technologies. Temporal responses, similar to persistence may still play a role in these new detectors.

\subsection{Phosphors for Scanning Systems}

Although any phosphor can potentially be used for scanning, the phosphor must exhibit several properties, it the system is to yield high quality images. The phosphor must have good $x$-ray absorption and it must have a very bright light output to make the most efficient use of signal 
available. Light spreading in the phosphor must be minimized to maintain the highest possible spatial resolution, and, as shown in Chapter II, the phosphor must have a rapid temporal response. An exhaustive analysis of every phosphor that might be suitable for a scanning system cannot be provided in this work. However, two commonly used phosphors will be described as an indication of the considerations involved in phosphor selection.

Phosphor screens made of $\mathrm{Gd}_{2} \mathrm{O}_{2} \mathrm{~S}$ : $\mathrm{Tb}$ are widely used in conventional radiography, and have been employed in several static (stationary) digital systems. This phosphor has several favourable advantages over other phosphors that makes it attractive for use in $\mathrm{x}$-ray imaging. This phosphor has a high density and high atomic number for good $x$-ray absorption (quantum efficiency)[30]. It also has a very high light output producing very high signal levels. Because of its good quantum efficiency and brightness, this phosphor can be made thinner than other phosphors. Lateral light spread from the phosphor is reduced if the phosphor is made as thin as possible. Thus, $\mathrm{Gd}_{2} \mathrm{O}_{2} \mathrm{~S}$ : $\mathrm{Tb}$ screens are designed to have a very good spatial resolution.

Because of its wide availability and good imaging characteristics, $\mathrm{Gd}_{2} \mathrm{O}_{2} \mathrm{~S}$ : Tb screens tend to be the first choice for prototype scanning systems. However, the loss in resolution due to phosphor persistence is considerable. As seen in Figure 2.9, the loss can be quite large, especially at high velocities. Even at a reasonable velocity of $40 \mathrm{~mm} / \mathrm{s}$, the MTF drops by $35 \%$ at $10 \mathrm{~mm}^{-1}$. If this phosphor is to be used, the scan velocity must be kept quite low.

One alternative phosphor that has been investigated for use in digital systems is the crystalline phosphor CsI:Tl. At first glance, CsI:Tl may seem to be a poor choice. This material is hydroscopic and can be damaged by water in the atmosphere. Thus, it is necessary to put a protective coating on the phosphor to protect it from damage. As well, the crystal is plastic at room temperature and it can be deformed and damaged easily. It also has a relatively low density $\left(4.52 \mathrm{~g} \cdot \mathrm{cm}^{-3}\right)[30]$ meaning that the crystal must be made very thick to achieve a high quantum efficiency. However, CsI:Tl does have some redeeming properties that makes it highly attractive for digital imaging. This phosphor can be grown or thermally cracked to produce a collimated 
crystal structure[49]. The collimated crystals act like fibre optics to channel the emitted light down through the crystal which limits lateral light spread. Thus, spatial resolution can be kept quite high, even when the crystals are made very thick to achieve high quantum efficiency. As seen in Chapter II Table 2.2, CsI:Tl is dominated by a very short time constant $(2.9 \mu \mathrm{s})$, which means that blurring due to phosphor persistence will be minimal. It should be noted, however, that the CsI:Tl tested in Chapter II was a bulk single crystal. The cracked or collimated crystals will have a significantly increased number of electron trap due to the increased surface area. This may mean that afterglow may be much higher in the collimated crystals used for the scanning system.

Other phosphors may be good choices for use with scanning detectors. Although it is desirable to have high values of spatial resolution, quantum efficiency, and light output as well as rapid temporal response, there are trade-offs between these requirements. To maintain high quantum efficiency, the phosphors must be made thick, but more light spreading occurs as thickness increases, reducing spatial resolution. There is also a compromise between brightness and temporal response; the brightest phosphors tend to have longer lifetimes[35, 31]. As mentioned brietly in Chapter I, electrons return to the ground state by luminescence emission or possibly by competitive radiationless transitions. If the radiationless transition probability is high, the excited state will de-populate much faster yielding a smaller lifetime for the excited state. However, fewer electrons will be available for the luminescence transition which reduces the brightness. On the other hand, the brightest phosphors have a much lower probability of a radiationless transition, allowing more electrons to undergo light emission, but with a longer lifetime in the excited state. This brightnesslifetime relationship is not always true, but certainly the two slowest phosphors measured in chapter II were also the brightest $\left(\mathrm{Gd}_{2} \mathrm{O}_{2} \mathrm{~S}: \mathrm{Tb}\right.$, and $\left.\mathrm{ZnCdS}: \mathrm{Ag}\right)$. 


\subsection{Effect of Phase Shift on Image Quality in X-ray Imaging}

It was demonstrated in Section 2.2.1 that the the phase term in the Fourier transform of the PSF can significantly affect resolution. In systems in which the PSF is symmetrical, the MTF contains all the information required to construct an image. However, an asymmetrical PSF requires the complete OTF to fully characterize it. If an estimate of resolution was made on a system with an asymmetrical PSF based solely on MTF information, the result can be underestimated. The size of the underestimate will depend on the degree of asymmetry in the PSF. For systems in which the PSF is only marginally asymmetric, the MTF (alone) would provide a reasonable measure of resolution.

Consider a very simple phosphor coupled scanning system in which all blurring mechanisms (except phosphor persistence) contribute to form a PSF with a Gaussian profile:

$$
\operatorname{PSF}_{g}(x)=e^{-\frac{1}{2}\left(\frac{x}{3}\right)^{2}}
$$

where $s$ is the standard deviation of the Gaussian which defines its width. This has an OTF which is:

$$
O T F_{g}(x)=\sqrt{2 \pi s^{2}} e^{-2(\pi \cdot s \cdot f)^{2}}
$$

Now assume that this system uses a scanning phosphor coupled detector. The PSF of this system is the convolution of Equation 2.1 and Equation 3.1:

$$
P S F(x)=P S F_{g}(x) * P S F_{p}(x)
$$

Hence, the total OTF for the system is :

$$
\begin{aligned}
O T F(f) & =O T F_{g}(f) \cdot O T F_{p}(f) \\
& =k \cdot e^{-2(\pi \cdot s \cdot f)^{2}} \frac{1}{1+i \cdot 2 \pi v \tau f}
\end{aligned}
$$


where $k$ represents the product of the normalization constants of the two OTFs.

If the MTF (magnitude of Equation 3.4) was used instead of the complete OTF, this corresponds to an incorrect PSF. Using only the MTF, the resolution can be overestimated. This is demonstrated in Figure 3.1. On the left side of the figure, a very high resolution system with Gaussian blur, $s=5 \mu \mathrm{m}$ and a phosphor with lifetime, $\tau=500 \mu \mathrm{s}$ is considered. Figure 3.1A is a plot of the PSF for the static case $\left(P S F_{g}(x)\right)$ by the dotted line and the true PSF $(P S F(x))$ by the solid line. Also shown is the incorrect PSF (dashed line), determined by the inverse Fourier transform of the magnitude of Equation 3.4. These plots are shown for a scanning velocity of $100 \mathrm{~mm} / \mathrm{s}$. As a simple measure of resolution, the full-width half maximum (FWHM) was was determined from both PSFs. By comparing the true FWHM of $P S F(x)$ to the incorrectly determined PSF, the effect of the phase term can be seen. Figure $3.1 \mathrm{C}$ is a plot of the error in the FWHM measurement as a function of scanning velocity.

Figure 3.1B is the same phosphor $(\tau=500 \mu \mathrm{s})$, but with lower static resolution $(s=30 \mu \mathrm{m})$. The relative significance of phosphor persistence is greatly reduced. Note that the position of $P S F_{T}(x)$ is still shifted, but the asymmetry is much less noticeable. The error in the FWHM measurement between the correct PSF and the incorrect one is also quite small. Because the static blurring is much greater, the additional loss of resolution due to the distortion induced by the phase term is very small.

If the resolution for the best commercial screens currently available was modeled by a Gaus$\operatorname{sian}, s$ would be in the range of $20-40 \mu \mathrm{m}$. Based on Figure 3.1D, the resolution will not be greatly affected by the phase component of the OTF. The MTF is therefore sufficient for quantifying resolution. Note, however, that the true shape and shift induced by phosphor persistence is still different than predicted by the incorrect PSF.

There may be other mechanisms that can produce asymmetric PSF in various $x$-ray imaging systems. Certainly, any system that uses a scanning acquisition may be susceptible to phase distortion. For example, systems that use laser scanning such as film digitizers, or photostimulable 


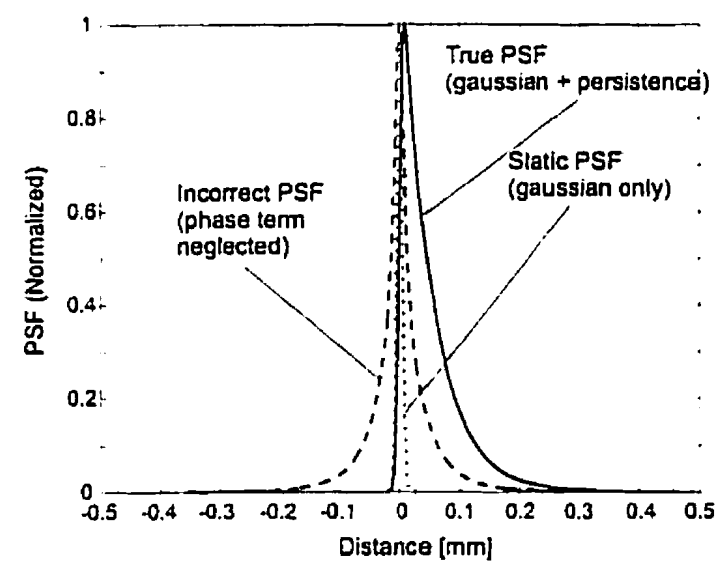

A
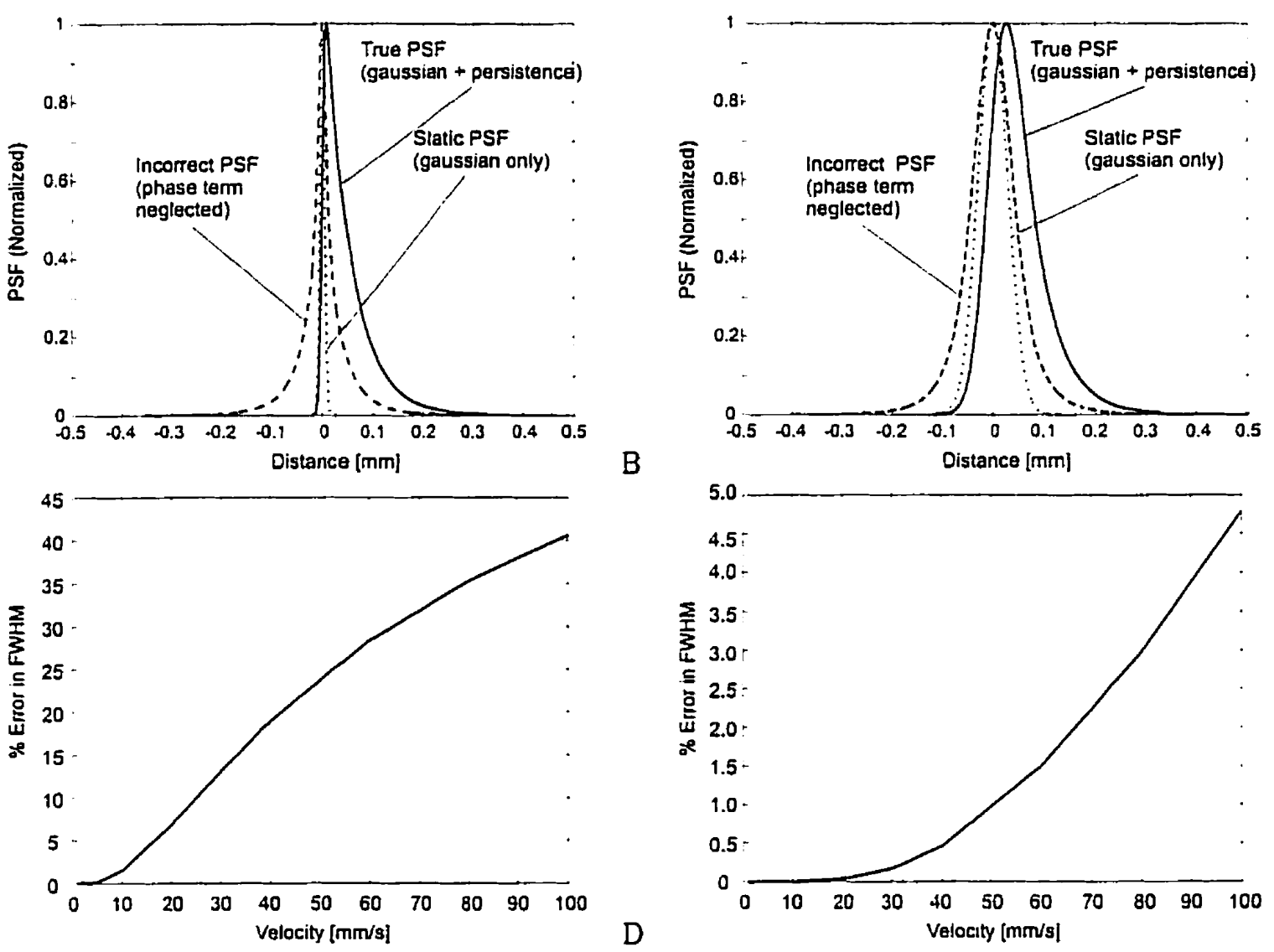

B

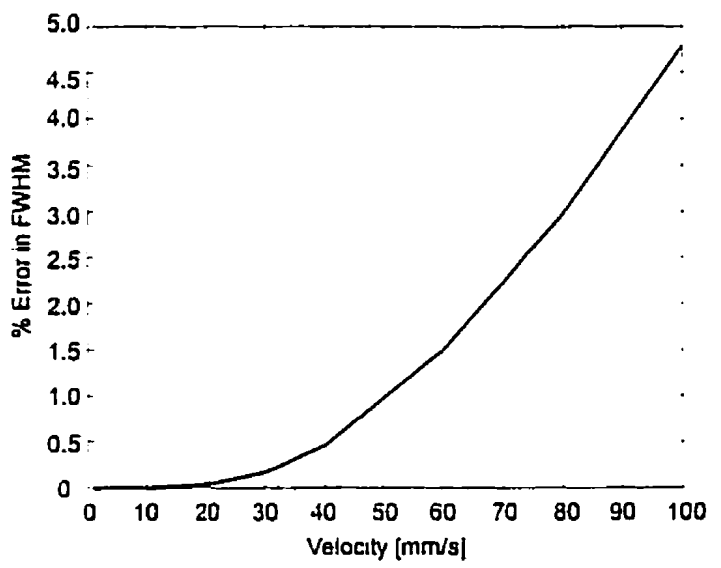

Figure 3.1: Dllustration of the impact of phase on high resolution system (A) and low resolution system (B). C and D show the error resulting in determining the FWHM of the point spread function if the incorrect PSF is used. 
phosphor systems, may show a lag in the response time of the detection electronics. If these effects are the dominant blurring mechanism, the full OTF must be used to describe resolution.

\subsection{Phosphor Alternatives-Direct Conversion Systems}

The phosphor acts as a conversion stage from x-rays to light which is, in turn, detected by a photosensitive detector. Because of the tradeoff between resolution (thin phosphors) and high efficiency, the phosphor is limited in its image quality. There are alternative detectors that can potentially exceed the image quality of phosphor coupled systems: direct conversion detectors.

A direct conversion detector is a device that absorbs $\mathrm{x}$-rays and converts them directly to an electronic charge signal. Like phosphor coupled systems, the detector replaces the film-screen cassette of a conventional radiography unit. The detector is a semiconductor in which the absorbed $x$-ray liberates electron-bole pairs (EHP) from the atoms in the detector material. The EHPs are collected by electrodes on the opposite surfaces of the semiconductor. The detectors use a high voltage potential across each pixel element to create a strong electric field that confines EHP migration to within a single pixel only. Because of this, the detectors can be made very thick to have a high $\mathrm{x}$-ray absorption efficiency while maintaining a high resolution due to the confinement of EHP migration. Zhao et al. have demonstrated for an amorphous selenium prototype, that resolutions are limited only by the pixel pitch and high efficiencies are possible[7]. The detectors can be made of many materials, including selenium[7], silicon[50], and zinc cadmium telluride $(\mathrm{ZnCdTe})[51]$.

Although eliminating the phosphor would eliminate the resolution-efficiency tradeoffs, temporal responses may still be significant. If a CCD "style" detector is used, charge transfer inefficiency will be present (Equation 1.7). An active matrix amorphous selenium detector has also demonstrated a small lag effect associated with incomplete photoconductive charge collection[7](i.e. collection of the EHPs liberated by the absorption of an $\mathrm{x}$-ray). If the direct conversion system is 
designed to be a scanning system, then these temporal phenomena will produce one-sided (asymmetrical) blurring. This will require the use of a complex OTF rather than the MTF in accurate characterization of resolution. There is a potential for extremely good stationary resolution. Similar to the case for the hypothetical scanning system associated with in Figure 3.1A, the temporal response could be the dominant blurring mechanism. If this is the case, the complex OTF describes greater resolution loss than the MTF predicts.

The direct conversion systems demand extremely advanced technology, from fabrication of high purity semiconductors to fast low noise electronics. Because these technological hurdles are only now being reached, a commercial direct conversion radiographic system will not be readily available for several years. Until then, phosphor systems will play an important role in the development of digital $x$-ray imaging as an established modality.

\subsection{Summary}

There are image quality limitations in film-screen radiography, and alternative image acquisitions systems are being developed. One type of system is an $x$-ray slot scanning digital detector. Because of the motion involved the image acquisition process, there is the potential for additional sources of blur. One such source is due to the persistence of light emission from the phosphor used in the detector. A model was developed for the resolution losses due to phosphor persistence based on the scanning velocity and the behaviour of the phosphor persistence. This model was shown to produce an asymmetrical blur in the image, requiring a complex OTF for accurate characterization, rather than relying solely on the MTF which has been conventionally used in describing resolution for $\mathrm{x}$-ray imaging. The phase component of the OTF was shown to cause distortions that could cause an apparent resolution loss in addition to the resolution loss described by the MTF. This additional loss was most noticeable when phosphor persistence was the dominant blurring mechanism in the imaging system. Accurate knowledge of the temporal response of the phosphor was required for the 
model and the response was measured for eight commercially available phosphors. A pulse sampling apparatus was used to measure the temporal response. This required a technique to correct for the influence of the $\mathrm{x}$-ray pulse shape on the apparent phosphor response. An iterative deconvolution method was used to correct for x-ray pulse-shape distortion. Both the primary luminenscence and the long term afterglow were measured. The phosphor, $\mathrm{Gd}_{2} \mathrm{O}_{2} \mathrm{~S}$ : $\mathrm{Tb}$, had the slowest primary luminescence, which would cause the greatest blurring in a scanning system. All the phosphors demonstrated a certain amount of afterglow, and at least two phosphors ( $\mathrm{ZnCdS}: \mathrm{Ag}$, and $\mathrm{YTaO}_{4}$ ) demonstrated an afterglow level that was large enough to reduce resolution and contrast. Using the knowledge obtained from the luminescence measurements, a phosphor can be selected that permits the highest resolution for a slot scanning system. One of the possible phosphors is CsI:Tl which was shown to have a very short primary luminescence, and a reasonably low afterglow level. This phosphor can be made in a collimated structure that produces high resolution. Despite concerns about its fragility and sensitivity to humidity, this phosphor seems to be a good choice for scanning detectors. In the future, phosphor coupled systems may be replaced by direct conversion detectors which potentially offer higher resolution and better sensitivity. The temporal response of these detectors may play a role in resolution, in a similar manner to the effect of phosphor persistence on image quality. However, these detectors are still several years from practical use. Until then, phosphor coupled systems will play a major role in the establishment of digital radiography in the clinical setting. 


\section{Appendix A}

\section{Description of Some Common}

\section{Phosphors}

This section will give a brief description of some phosphors that are used in $x$-ray imaging. These phosphors have demonstrated particular properties such as good light output and conversion efficiency, spatial resolution, or spectral matching with film to be potentially used in a radiography system. Unless otherwise noted, most of the information on the following materials is obtained from Blasse and Grabmaier[30].

\section{BaFCl:Eu}

This phosphor has a layered crystal structure, which makes the crystals plate-like and very aniostropic, resulting in the tendency to pipe light towards the sides of the plates. The density is relatively low, $4.56 \mathrm{~g} \cdot \mathrm{cm}^{-3}$. requiring a thicker screen than some of the other phosphors (which in turn leads to additional light spreading and hence lower spatial resolution). The spectrum is a band emission with a maximum at $380 \mathrm{~nm}$ (blue). Because of its relatively low density, it has been replaced by modern rare earth phosphors like $\mathrm{Gd}_{2} \mathrm{O}_{2} \mathrm{~S}$ : Tb in radiographic screen use.

\section{LaOBr:Tm}

This phosphor has the same structure as $\mathrm{BaFCl}$ but a higher density $\left(6.13 \mathrm{~g} \cdot \mathrm{cm}^{-3}\right)$. The 
spectrum consists of narrow lines as a result of the transitions of $\mathrm{Tm}^{3+}$ in the near $\mathrm{u}-\mathrm{v}$ and blue. $\mathrm{Gd}_{2} \mathrm{O}_{2} \mathrm{~S}: \mathrm{Tb}$

Again this phosphor has a layered crystal structure. This phosphor has an excellent conversion efficiency, $\eta_{c}=15 \%$. Conversion efficiency is the ratio of the total energy of the output light photons to the total energy of an input $x$-ray. This phosphor also has a very high density of $7.34 \mathrm{~g} \cdot \mathrm{cm}^{-3}$. The spectrum consists of narrow lines centred on $550 \mathrm{~nm}$ (green). This phosphor has become the most popular phosphor in mammography and general radiography.

$\mathrm{YTaO}_{4}$

This phosphor has a similar crystal structure to $\mathrm{CaWO}_{4}$, which was historically used in the first radiographic screens. However, $\mathrm{YTaO}_{4}$ has a much higher density, $7.55 \mathrm{~g} \cdot \mathrm{cm}^{-3}$ and a reasonably good conversion efficiency $\eta_{c}=9 \%$. It has a band spectrum with a peak at $350 \mathrm{~nm}$ (blue).

$\mathrm{Gd}_{2} \mathrm{O}_{2} \mathrm{~S}: \mathrm{Pr}$

Because the host crystal is the same as $\mathrm{Gd}_{2} \mathrm{O}_{2} \mathrm{~S}$ : Tb, the density is the same $\left(7.34 \mathrm{~g} \cdot \mathrm{cm}^{-3}\right)$ and it has the same crystal structure. However the emission spectra associated with the $\mathrm{Pr}^{3+}$ ion is broad band rather than narrow lines, and it has a very short decay time compared to $\mathrm{Tb}^{3+}$.

\section{CsI:Tl}

This has a cubic crystal structure, with average density $\left(4.52 \mathrm{~g} \cdot \mathrm{cm}^{-3}\right)$, and a short primary luminescence. The emission spectra is very broad band extending from 400 to $700 \mathrm{~nm}$ giving rise to a white light emission. At room temperature, the crystal is plastic (deformable) and mildly hydroscopic which can damage its luminescence properties.

$\mathrm{Bi}_{4} \mathrm{Ge}_{3} \mathrm{O}_{12}$ (BGO) Ref. [36]

This is a scintillator that is very commonly used in particle physics. It has a very short lifetime (300 ns) and low afterglow $(0.005 \%$ after $3 \mathrm{~ms})$. The conversion efficiency is low with a maximum of $6 \%$ at low temperature (2\% at room temperature) and it has a high density ( 7.13 $\mathrm{g} \cdot \mathrm{cm}^{-3}$ ). The light output is broadband centered at $480 \mathrm{~nm}$. This is not used for radiographic screens, rather, it is used as a single crystal for photon or particle counting physics. 
ZnCdS:Ag(Cl) Ref. [35]

This phosphor is one of the brightest phosphors, with a conversion efficiency up to $\eta_{c}=23 \%$. This phosphor is a compensated semiconductor containing both donor (Cl) and acceptor ( $\mathrm{Ag}$ ) dopants. The relative proportion of zinc to cadmium can vary, which changes the color of the emission spectrum from yellow to green. The brightest is $\mathrm{Zn}_{0.70} \mathrm{Cd}_{0.30} \mathrm{~S}: \mathrm{Ag}(\mathrm{Cl})$. Unfortunately, its density is low $\left(4.4 \mathrm{~g} \cdot \mathrm{cm}^{-3}[46]\right)$. This type of phosphor was used in early image intensifiers because of the high level of light output. 


\section{Appendix B}

\section{Levenberg-Marquhardt Non-Linear}

\section{Least Squares}

The following section will give a brief description of the non-linear least square technique used in this work. The reader is referred elsewhere $[39,52,40]$ for a more complete description.

Given a set of data, $\left\{x_{i}, y_{i}\right\}$ containing $m$ points with errors on the $y$ values of $\sigma_{i}$, and a model equation, $y_{0}\left(x_{i}, a_{k}\right)$ which has a set of $N$ coefficients, $a_{k}$, a mean squared difference can be defined:

$$
\chi^{2}=\sum_{i=1}^{m}\left\{\frac{1}{\sigma_{i}^{2}}\left[y_{i}-y_{0}\left(x_{i}\right)\right]^{2}\right\}
$$

For some starting value for the coefficients, $\left\{a_{k}\right\}$, an initial chi-square, $\chi_{0}^{2}$ is calculated. We need to find a set of updates, $\left\{\delta a_{k}\right\}$ that will yield the minimum chi-square value. In other words, we need find $\delta a_{k}$ that will produce a 0 for the derivative of the chi-square:

$$
\frac{\partial \chi^{2}}{\partial a_{k}}=0
$$

Performing a first order Taylor expanson of Equation B.2 yieids: 


$$
\frac{\partial \chi^{2}}{\partial a_{k}} \approx \frac{\partial \chi_{0}^{2}}{\partial a_{k}}+\sum_{j=1}^{N}\left(\frac{\partial^{2} \chi_{0}^{2}}{\partial a_{j} \partial a_{k}} \delta a_{j}\right)=0
$$

To simplify the equations, some new terms are defined:

$$
\beta_{k}=-\frac{1}{2} \frac{\partial \chi_{0}^{2}}{\partial a_{k}}
$$

and, the curvature matrix, $\alpha$ :

$$
\alpha_{j k}=\frac{1}{2} \frac{\partial^{2} \chi_{0}^{2}}{\partial a_{j} \partial a_{k}}
$$

This reduces Equation B.3 to a much simpler form:

$$
-\beta_{k}+\sum_{j=1}^{N} \alpha_{j k} \delta a_{j}=0
$$

To solve for the updates, $\delta a_{j}$, we need to define an additional function, called the error matrix:

$$
\epsilon=\alpha^{-1}
$$

Solving of the updates from Equation B.6 yields:

$$
\delta a_{j}=\sum_{k=1}^{N}\left(\beta_{j} \epsilon_{j k}\right)
$$

The vector $\beta$ can be determined quite easily:

$$
\begin{aligned}
\beta_{k} & =-\frac{1}{2} \frac{\partial}{\partial a_{k}}\left[\sum_{i=1}^{m}\left\{\frac{1}{\sigma_{i}^{2}}\left[y_{i}-y_{0}\left(x_{i}\right)\right]^{2}\right\}\right] \\
& =\sum_{i=1}^{m}\left\{\frac{1}{\sigma_{i}^{2}}\left[y_{i}-y_{0}\left(x_{i}\right)\right] \frac{\partial y_{0}\left(x_{i}\right)}{\partial a_{k}}\right\}
\end{aligned}
$$

The elements of the curvature matrix are : 


$$
\begin{aligned}
\alpha_{j k} & =\frac{1}{2} \frac{\partial \chi_{0}^{2}}{\partial a_{j} \partial a_{k}} \\
& =-\frac{\partial}{\partial a_{j}}\left(\frac{1}{2} \frac{\partial}{\partial a_{k}}\left[\sum_{i=1}^{m}\left\{\frac{1}{\sigma_{i}^{2}}\left[y_{i}-y_{0}\left(x_{i}\right)\right]^{2}\right\}\right]\right) \\
& =\sum_{i=1}^{m}\left\{\frac{\partial y_{0}\left(x_{i}\right)}{\partial a_{j}} \frac{\partial y_{0}\left(x_{i}\right)}{\partial a_{k}}-\left[y_{i}-y_{0}\left(x_{i}\right)\right] \frac{\partial^{2} y_{0}\left(x_{i}\right)}{\partial a_{j} \partial a_{k}}\right\}
\end{aligned}
$$

Assuming that $\chi_{0}^{2}$ is near the actual minimum, the curvature calculation is simplified:

$$
\alpha_{j k} \approx \sum_{i=1}^{m}\left\{\frac{\partial y_{0}\left(x_{i}\right)}{\partial a_{j}} \frac{\partial y_{0}\left(x_{i}\right)}{\partial a_{k}}\right\}
$$

This works well when $\chi_{0}^{2}$ is near the actual minimum, but when it is far away, the updates, $\delta a_{j}$, are either over or underestimated. Hopefully, if the coefficients $a_{j}$ are updated, the resultant $\chi^{2}$ will be closer to the minimum and the whole process can be repeated until the updates yield a $\chi_{0}^{2}$ that is close to the minimum. However, convergence may be slow or never even occur with this method.

The Levenberg-Marquahardt (L-M) uses an additional parameter to help improve convergences when $\chi^{2}$ is far from the minimum. In the L-M technique, $\alpha$ is substituted with $\alpha^{\prime}$ which is has its diagonal elements increased by a factor, $\lambda$ :

$$
\alpha_{j k}^{\prime}= \begin{cases}\alpha_{j k}(1+\lambda) & \text { if } j=k \\ \alpha_{j k} & \text { otherwise }\end{cases}
$$

For very large $\lambda$, the technique becomes a gradient search or steepest descent method with updates in the direction of the gradient:

$$
\beta_{j} \approx \lambda \delta a_{j} \alpha_{j j}
$$

A gradient search technique works very well when $\chi_{0}^{2}$ is far from the minimum but very slowly when it approaches the shallow slopes near the minimum. Thus, if $\lambda$ is adjusted during the 
search, making it large when $\chi_{0}^{2}$ is far from the minimum (a gradient search) and small when it is close (an analytic search) the best of both algorithms can be used.

The parameter $\lambda$ can be updated in a number of ways, but a fairly robust techniques uses information about the linearity of $\chi^{2}$ space. A linear estimate of the new $\chi^{2}$ value is calculated:

$$
\chi_{L}^{2}=\sum_{i=1}^{m}\left\{\sum_{j=1}^{N}\left[\frac{\partial y_{0}}{\partial a_{j}} \delta a_{j}\right]+\frac{1}{\sigma^{2}}\left[y_{i}-y_{0}\right]^{2}\right\}
$$

A second estimate of $\chi^{2}$ is calculated by performing a cubic interpolation of with the $\chi^{2}$ obtained from the previous iteration. Let this interpolated value be $\chi_{C}^{2}$. The parameter $\lambda$ can be updated:

$$
\lambda^{\prime}= \begin{cases}\lambda+\frac{x_{C}^{2}-\chi_{L}^{2}}{q} & \text { if } \chi_{L}^{2}<\chi_{C}^{2} \\ \frac{\lambda}{1+q} & \text { otherwise }\end{cases}
$$

where $q$ is a 'stepsize' parameter which is also cubically interpolated from previous values of $\chi^{2}$. The use of $q$ provides a method to reduce the possibility of overshooting the minimum, giving more control over the iterative updates. The stepsize parameter is then used in the update of the coefficients:

$$
a_{k}^{\prime}=a_{k}+q \cdot \delta a_{k}
$$

This process is repeated, calculating a new $\chi^{2}$ and hence new $\lambda, q$ and $\delta a_{k}$ values until either the maximum number of allowed iterations is reached, the updates no longer improve $\chi^{2}$ or when the following tolerance conditions are satisfied:

$$
\begin{aligned}
\max \left|\delta a_{k}\right| & <T_{a} \\
\max \mid \sum \frac{1}{\sigma^{2}} \frac{\partial y_{0}}{\partial a_{k}}\left[y_{i}-y_{0}\right] ! & <10 \cdot(T a+T b) \\
2 \sum_{k=1}^{n}\left\{\sum \frac{1}{\sigma^{2}} \frac{\partial y_{0}}{\partial a_{k}}\left[y_{i}-y_{0}\right]\right\} \delta a_{k} & <T_{b}
\end{aligned}
$$


where $T_{a}$ and $T_{b}$ are tolerance values (set to 0.01 for this work). Thus, the largest update to the variables must be less than $T_{a}$, and the gradient of $\chi^{2}$-space must be smaller than $10 \cdot\left(T_{a}+T_{b}\right)$ and finally, the scalar product of $\delta a$ and $-\frac{\partial \chi^{2}}{\partial a}$ must be less than $T_{b}$. In other words both the gradient and the updates should be small when $\chi^{2}$ is at a minimum.

\section{B.1 Model Equations for Luminescence}

The model equations for $y_{0}$ and $\frac{\partial y_{0}}{\partial a_{k}}$ for primary luminescence were the following. Let $y_{0}$ be the pulse response, $R_{i}$ measured in the luminescence experiment, and $P_{i}$ is the $x$-ray pulse. The parameters to be fitted are $a_{1}=\tau_{1}, a_{2}=w_{1}, \ldots$ From Equation 1.15, and substituting $\left\{a_{j}\right\}$ for the parameters, then:

$$
R_{i}=P_{i} *\left[\sum_{j=1,3, \ldots}^{N} \frac{a_{j+1}}{a_{j}} e^{-\frac{t_{1}}{a_{j}}}\right]
$$

In order to numerically calculate the convolution, this can be done using trapezoidal integration, as suggested by Grinvald and Steinberg for luminescence analysis[38]:

$$
R_{i}=\Delta t \sum_{\xi=1}^{i} P_{\xi} \sum_{j=1,3, \ldots}^{N} \frac{a_{j+1}}{a_{j}} e^{-\frac{(i-\xi) \Delta t}{a_{j}}}
$$

where $\Delta t$ is the time between sample points. In order to reduce calculation time. Equation B.21 can be re-expressed as a recursive function[38]:

$$
R_{i}=\sum_{j=1,3, \ldots}^{N}\left(R_{i-1}+\frac{1}{2} \Delta t a_{j+1} P_{i-1}\right) e^{-\frac{\Delta t}{a_{1}}}+\frac{1}{2} \Delta t a_{j+l} P_{i}
$$

The partial derivatives of this equation is straightforward: 


$$
\frac{\partial R_{i}}{\partial a_{j}}= \begin{cases}\frac{\partial R_{i}}{\partial a_{j}}+\frac{\Delta t}{a_{j}^{2}}\left(R_{i-1}-\frac{1}{2} a_{j+1} P_{i-1}+\frac{1}{2} \Delta t \frac{a_{j+1}}{a_{j}} P_{i-1}\right) e^{-\frac{\Delta t}{a_{1}}}-\frac{1}{2} \Delta t \frac{a_{j+1}}{a_{j}^{2}} P_{i-1} & \text { if } j=1,3,5, \ldots \\ \frac{R_{i}}{a_{j}} & \text { if } j=2,4,6, \ldots\end{cases}
$$

These equations were used in the curve fitting described in Section 2.2.2 


\section{Bibliography}

[1] F. A. Mettler, Jr, J. E. Briggs, R. Carchman, K. K. Altobelli, B. L. Hart, and C. A. Kelsey, "Use of radiology in U.S. general short-term hospitals: 1980-1990", Radiology 189, 377-380 (1993).

[2] D. O. Weber, "Practice patterns", in Diagnostic Ultrasound: Marked Trands and Clinical Practice in the U.S., (Miller Freeman Inc., 1993), volume 2, p. 88.

[3] M. J. Yaffe, "Digital mammography", in Syllabus: A Categorical Course in Physics Technical A spects of Breast Imaging, edited by A. G. Haus and M. J. Yaffe, (RSNA, Oak Brook, IL, 1993), pp. 271-282.

[4] A. G. Haus, "Screen-film image receptors and film processing", in Syllabus: A Categorical Course in Physics Technical Aspects of Breast Imaging, edited by A. G. Haus and M. J. Yaffe, (RSNA, 1993), pp. 69-84.

[5] P. Bunch, "Analysis of the detective quantum efficiency of a radiographic screen-film combination", Journal of the Optical Society of America. A 4, 902-9 (1987).

[6] V. F. Andolina, S. Lille, and K. M. Willison, Mammographic Imaging: A Practical Guide, (J. B. Lippincott Company, Philadelphia, 1992).

[7] W. Zhao and J. A. Rowlands, "X-ray imaging using amorphous selenium: Feasiblity of a flat panel self-scanned detector for digital radiology", Medical Physics 22, 1595-1604 (1995).

[8] M. Sonoda, M. Takano, J. Miyahara, and H. Kato, "Computed radiography utilizing scanning laser stimulated Iuminescence".

[9] E. F. Eikenberry, M. W. Tate, D. H. Bilderback, and S. M. Gruner, "X-ray detectors: Comparison of film, storage phosphors and ccd detectors", in Conference on Photoelectronic Image Devices, IOP Publishing, 1992.

[10] S. A. Feig and M. J. Yaffe, "Digital mammography, computer-aided diagnosis, and telemammography", Radiologic Clinics of North America 33, 1205-1230 (1995).

[11] Y. Jiang, R. M. Nishikawa, D. E. Wolverton, C. E. Metz, M. L. Giger, R. A. Schmidt, C. J. Yuborny, and K. Doi, "Malignant and benign clustered microcalcifications: Automated feature analysis and classification", Radiology 198, 671-678 (1996). 
[12] J. C. Dainty and R. Shaw, Image Science, (Academic Press, 1974).

[13] M. R. Spiegel, Theory and Problems of Complex Variables, (McGraw-Hill Book Company, New York, 1981).

[14] L. E. Antonuk, J. Boudry, W. Huang, D. L. McShan, E. J. Morton, J. Yorkston, M. J. Longo, and R. A. Street, "Demonstration of megavoltage and diagnostic $x$-ray imaging with hydrogenated amorphous silicon arrays", Medical Physics 19, 1455-1466 (1992).

[15] D. Sashin, B. S. Slasky, and C. R. Pearsall, "Reduced dose and improved image quality with a computerized line-scan radiography system", IEEE (1993).

[16] A. D. A. Maidment and M. J. Yaffe, "Analysis of the spatial-frequency dependent DQE of optically coupled digital mammography sources", Medical Physics 21 (1994).

[17] A. J. Wagner, "Contrast and grid performance in mammography", in Screen Film Mammography, (Medical Physics Publishing, Madison, Wisconsin, 1991), p. 124.

[18] R. Fahrig, J. G. Mainprize, N. Robert, A. Rogers, and M. J. Yaffe, "Performance of glass fiber antiscatter devices at mammographic energies", Mecial Physics 21, 1277-1282 (1994).

[19] T. T. Thompson, A Practical Approach to Modern X-ray Equipment. (Little, Brown and Company (Inc), Boston, 1978).

[20] G. T. Barnes and G. D. Frey, editors, Screen Film Mammography, (Medical Physics Publishing, Madison, Wisconsin, 1991).

[21] A. Maidment, Scanned-Slot Digital Mammography, PhD thesis, University of Toronto, 1993.

[22] D. W. Holdsworth, R. K. Gerson, and A. Fenster, "Scanned projection radiography with a slot beam", Proc. SPIE 914, 246-252 (1988).

[23] E. L. Dereniak and D. G. Crowe, Optical Radiation Detectors, (John Wiley \& Sons, 1984).

[24] T. S. Lomheim, L. W. Schumann, R. M. Shima, J. S. Thompsons, and W. F. Woodward, "Electro-optical hardware considerations in measuring the imaging capability of scanned timedelay-and-integrate charge-coupled imagers", Optical Engineering 29, 911-927 (1990).

[25] A. J. P. Theuwissen, Solid-State Imaging with Charge-Couple Devices, (Kluwer Academic Publishers, Netherlands, 1996).

[26] A. K. Cherri, A. A. S. Awwal, M. A. Karim, and D. L. F. Moon, "Restoration of moving binary images degraded owing to phosphor persistence", Applied Optics 30, 3734-3739 (1991).

[27] B. R. Sandel, D. F. Collins, and A. L. Broadfoot, "Effect of phosphor persistence on photometry with image intensifiers and integrating readout devices", Applied Optics 25, 3697-3703 (1986).

[28] H. E. Johns and J. R. Cunningham, The Physics of Radiology, (Charles C Thomas, Ilinois, 1983), p. 262, 4th edition. 
[29] D. V. O'Connor and D. Phillips, Time-correlated Single Photon Counting, (Academic Press, London, 1984).

[30] G. Blasse and B. C. Grabmaier, Luminescent Materials, (Springer-Verlag, 1994).

[31] M. D. Galanin, Luminescence of Molecules and Crystals, (Cambridge Interantional Science Publishing, Cambridge, England, 1996).

[32] D. Curie, Luminescence in Crystals, (Methuen, London, 1963).

[33] J. A. Shepherd, S. M. Grune, M. W. Tate, and M. Tecotzky, "A study of persistence in gadolinium oxysulfide x-ray phosphors", Proc. SPIE 2519, 24-30 (1995).

[34] B. C. Grabmaier, "Luminescent materials for medical application", Journal of Luminescence $60 / 61,967-970$ (1994).

[35] L. Ozawa, Cathodoluminescence, (Kodansha Ltd, Tokyo, 1990).

[36] G. F. Knoll, Radiation Detection and Measurement, (John Wiley \& Sons, New York, 1989), 2nd edition.

[37] M. P. Fogarty, C. N. Ho, and I. M. Warner, "Data handling in Huorescence spectrometry": in Optical Radiation Measurements: Measurement of Photoluminescence, edited by $\mathrm{K}$. D. Mielenz, (Academic Press, 1982), volume 3, chapter 7, pp. 263-269.

[38] A. Grinvald and I. Z. Steinberg, "On the analysis of thuorescence decay kinetics by the method of least-squares", Analytical Biochemistry 59, 583-598 (1974).

[39] P. R. Bevington, Data Reduction and Error Analysis for the Physical Sciences, (McGraw-Hill Book Company, New York, 1969), pp. 204-246.

[40] N. R. Draper and H. Smith, Applied Regression Analysis, (John Wiley \& Sons, New York, 1981), 2nd edition.

[41] A. V. Oppenheim and R. W. Schafer, Discrete-Time Signal Processing, (Prentice-Häll, Toronto, 1989).

[42] G. J. Berzins, A. H. Lumpkin, and H. L. Smith, "Characterization of tluorescent screens for imaging applications with $\mathrm{MeV}$ neutrons and photons", Optical Engineering 22, 633-642 (1983).

[43] V. Perez-Mendez, "Charged particle, gamma ray, and light detection in amorphous silicon devices", in Amorphous and Microcrystalline Semiconductor Devices: Optoelectronic Devices: edited by J. Kanicki. (Artech House, Boston, 1991).

[44] J. B. Birks, Theory and Practice of Scintillation Counting, (MacMillan Company, New York, 1964). 
[45] P. Schotanus, R. Kamermans, and P. Dorenbos, "Scintillation characteristics of pure and Tl-doped CsI crystals", IEEE Transactions on Nuclear Science 37, 177 (1990).

[46] B. A. Arnold, "Physical characteristics of screen-film combinations", in The Physics of Medical Imaging: Recording System Measurements and Techniques, edited by A. G. Haus, (AAPM, 1979).

[47] N. Bromberg, "Image intensifier contrast ratio measurements", in Acceptance Testing of Radiological Imaging Equipment, (ACR, 1982), pp. 45-52.

[48] R. Luhta and J. A. Rowlands, "Origins of flare in x-ray image intensifiers", Medical Physics 17, 913-921 (1990).

[49] I. Fujieda, G. Cho, J. Drewery, T. Gee, T. Jing, S. N. Kaplan, V. Perez-Mendez, and D. Wildermuth, "Radiation detection with evaporated CsI(Tl) coupled to a-Si:H photodioe layers", Materials Research Society Symposium Proceedings 219, 191-196 (1991).

[50] J. Henry, M. J. Yaffe, J. Venzon, F. Augustine, and T. O. Tumer, "Solid state $x$-ray detectors for digital mammography", Proc. SPIE 2432 (1995).

[51] J. F. Butler, S. J. Friesenhahn, C. Lingren, B. Apotovsky, F. P. Doty, W. L. Ashburn, and W. Dillon, "Cd $\mathrm{Cd}_{l-x} \mathrm{Zn}_{x}$ Te detector imaging array", Proc. SPIE 1896 (1993).

[52] A. Grace, Optimization Toolbox:User's Guide, (The Math Works Inc, Massachusetts, 1995). 
